\title{
Biogenesis of respiratory chain: Rcf1 and Rcf2 as a novel assembly factors
}

\author{
Dissertation \\ for award of the degree \\ "Doctor rerum naturalium" \\ at the Georg-August-Universität Göttingen \\ submitted by \\ Milena Vukotic \\ from Belgrade, Serbia
}

Göttingen, 2012 
Member of the Thesis Committee (First Reviewer):

Prof. Peter Rehling

Department of Biochemistry II

Centre for Biochemistry and Molecular Cell Biology

Georg-August-Universität, Göttingen

Member of the Thesis Committee (Second Reviewer):

Prof. Gerhard Braus

Department of Molecular Microbiology and Genetics

Institute for Microbiology and Genetics

Göttingen

Member of the Thesis Committee:

Prof. Dirk Görlich

Department Cellular Logistik

MPI for Biophysical Chemistry

Göttingen

Date of oral examination: 07.06.2012 
Herewith I declare, that I prepared this thesis on my own and with no other sources and aids than quoted.

Milena Vukotic 
The results presented in this thesis have been published previously in the following publications:

Milena Vukotic, Silke Oeljeklaus, Sebastian Wiese, F. Nora Vögtle, Chris Meisinger, Helmut E. Meyer, Anke Zieseniss, Doerthe M. Katschinski, Daniel C. Jans, Stefan Jakobs, Bettina Warscheid, Peter Rehling, Markus Deckers (2012) Rcf1 Mediates Cytochrome Oxidase Assembly and Respirasome Formation, Revealing Heterogeneity of the Enzyme Complex, Cell Metabolism 15:336-47

Alwaleed K. Alkhaja, Daniel C. Jans, Miroslav Nikolov, Milena Vukotic, Oleksandr Lytovchenko, Fabian Ludewig, Wolfgang Schliebs, Dietmar Riedel, Henning Urlaub, Stefan Jakobs, and Markus Deckers (2012) MINOS1 is a conserved component of mitofilin complexes and required for mitochondrial function and cristae organization, Mol Biol Cell, 23:247-57

David U. Mick, Milena Vukotic, Heike Piechura, Helmut E. Meyer, Bettina Warscheid, Markus Deckers, and Peter Rehling (2010) Coa3 and Cox14 are essential for negative feedback regulation of COX1 translation in mitochondria, $\mathrm{J}$ Cell Biol 191:141-54 


\section{Table of contents}

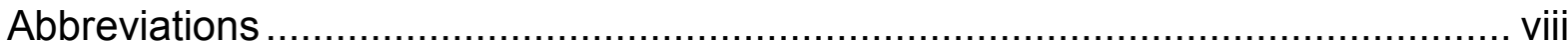

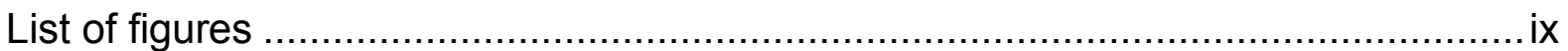

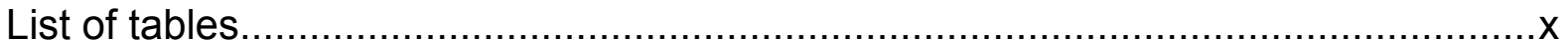

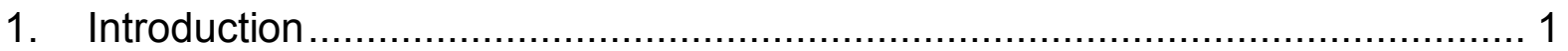

1.1. Mitochondria ................................................................................ 1

1.2. Oxidative phosphorylation and the electron transport chain ....................... 2

1.3. Ubiquinol-cytochrome $\mathrm{c}$ reductase ...................................................... 3

1.4. Cytochrome oxidase......................................................................... 4

1.4.1. Composition of the cytochrome oxidase is oxygen-dependent ............. 5

1.4.2.1. Import of mitochondrial proteins ................................................ 6

1.4.2.1.1. The translocase of the outer mitochondrial membrane ........... 7

1.4.2.1.2. The presequence translocase of the inner mitochondrial

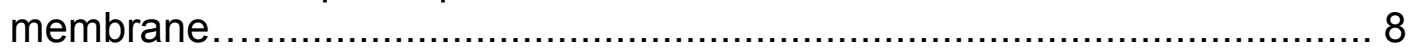

1.4.2.2. Export of mitochondrial-encoded proteins ….............................. 9

1.4.2.3. Assembly of cytochrome oxidase ........................................... 9

1.5. Respiratory chain supercomplexes ……............................................ 10

1.5.1. Diversity of respiratory supercomplexes......................................... 11

1.5.2. Functional and structural significance of supercomplex formation ....... 13

1.5.3. Formation of respiratory supercomplexes ........................................ 14

1.5.4. Interaction partners of respiratory supercomplexes ……................... 15

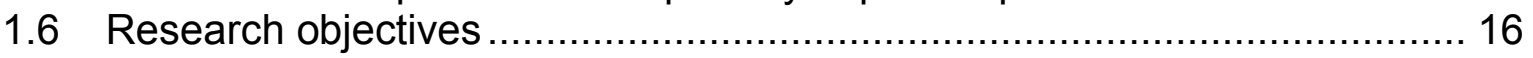

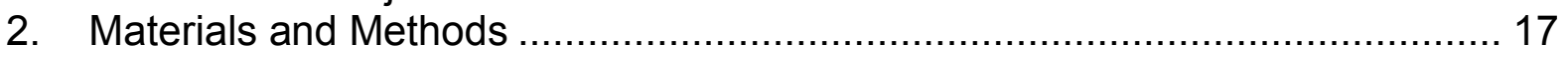

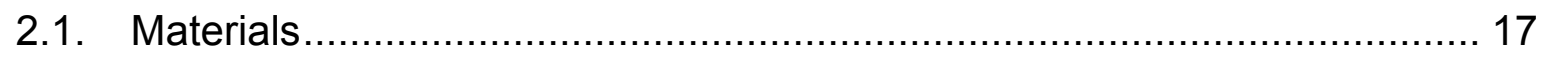

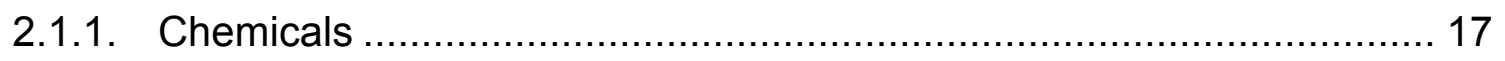

2.1.2. Enzymes, plasimds and kits.......................................................... 17

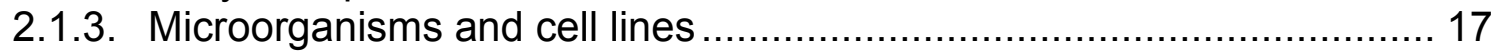

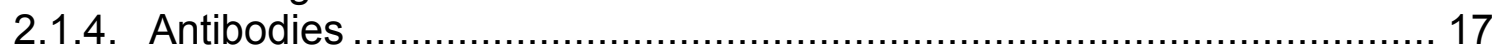

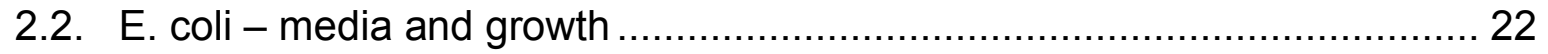

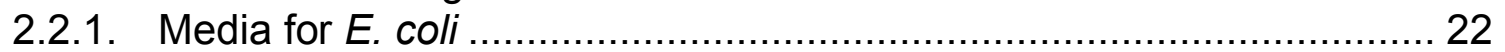

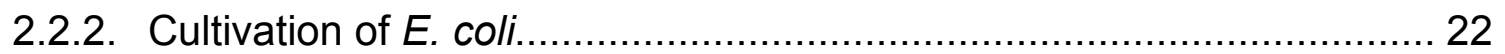

2.3. S. cerevisiae - media and growth .................................................. 22

2.3.1. Media for S. cerevisiae............................................................. 22

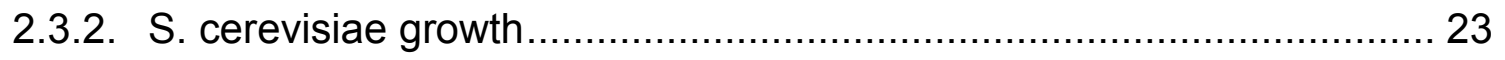

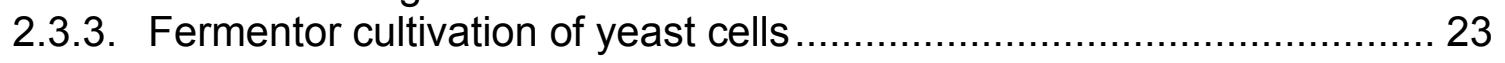

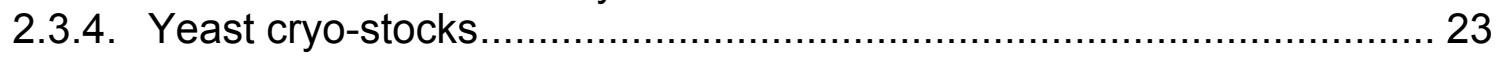

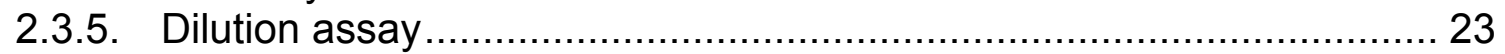

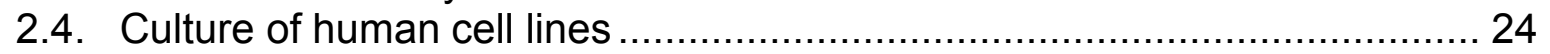

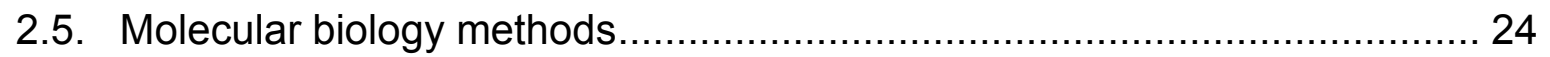

2.5.1. Determination of nucleic acid concentrations ................................... 24

2.5.2. DNA amplification by Polymerase Chain Reaction (PCR) ................... 24

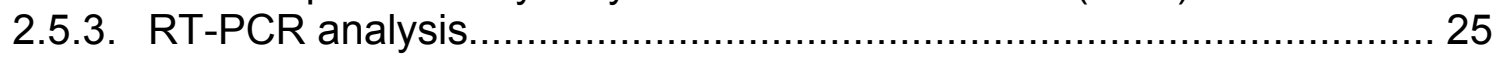

2.5.4. Agarose gel DNA electrophoresis ............................................... 25

2.5.5. Isolation of DNA from agarose gels............................................... 26

2.5.6. Enzymatic manipulation of DNA ……………................................. 26

2.5.7. Transformation of $E$. coli cells ........................................................ 26 
2.5.7.1. Preparation of transformation competent E. coli cells with $\mathrm{CaCl}_{2} . .26$

2.5.7.2. Transformation of $\mathrm{CaCl}_{2}$-competent $E$. coli cells by heat shock... 27

2.5.8. Sequencing of DNA............................................................... 27

2.5.9. Transformation of $S$. cerevisiae cells by lithium acetate method........... 27

2.5.10. Preparation of yeast genomic DNA ............................................. 28

2.5.10.1. Small scale preparation of yeast genomic DNA ....................... 28

2.5.10.2. Large scale preparation of yeast genomic DNA ....................... 28

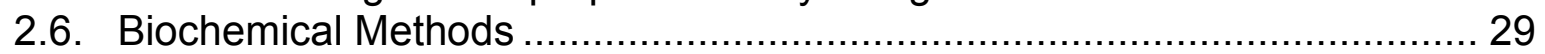

2.6.1. Determination of protein concentrations.................................... 29

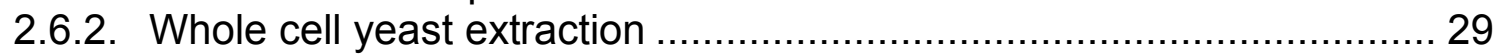

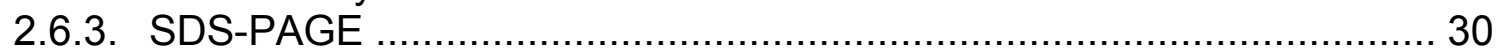

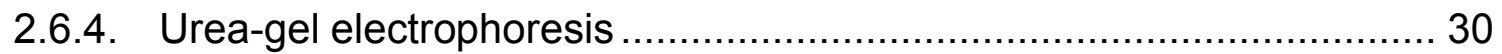

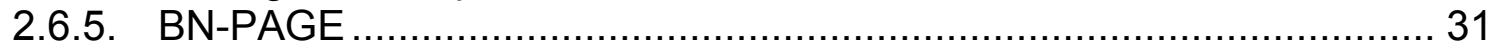

2.6.6. Transfer of proteins to PVDF membrane (Western-blotting) ............... 31

2.6.7. Protein detection techniques........................................................ 32

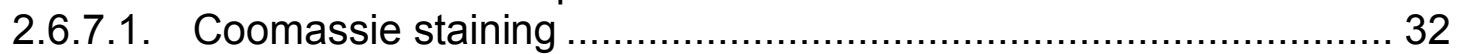

2.6.7.2. Gel staining with colloidal Coomassie Brilliant Blue..................... 32

2.6.7.3. Immunodetection of proteins on PVDF membranes ................... 32

2.6.7.4. Detection of radiolabelled proteins by autoradiography ............... 33

2.6.7.5. Mass spectrometry and data analysis....................................... 33

2.6.8. In vitro determination of enzyme activities .................................. 34

2.6.8.1. Malate dehydrogenase assay......................................... 34

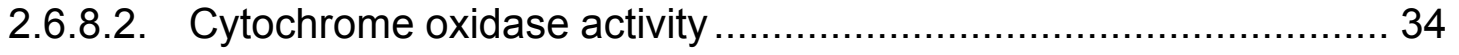

2.6.8.3. NADH-cytochrome c reductase activity ............................... 34

2.6.8.4. Aconitase activity ........................................................... 35

2.6.9. Oxygen consumption measurements....................................... 35

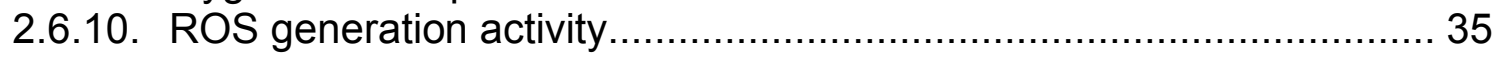

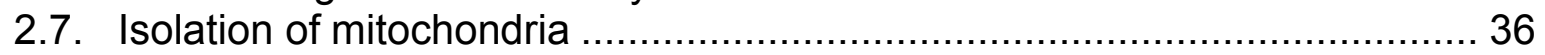

2.7.1. Isolation of mitochondria from S. cerevisiae ................................ 36

2.7.2. Isolation of mitochondria from human cells ................................. 37

2.8. In vitro import analysis in isolated mitochondria ............................... 37

2.8.1. In vitro synthesis of radiolabeled mitochondrial proteins ................... 37

2.8.1.1. In vitro transcription ....................................................... 37

2.8.1.2. In vitro translation ........................................................... 37

2.8.2. In vitro import of radiolabeled preproteins into isolated mitochondria.... 38

2.9. Localisation and topology of mitochondrial proteins ............................ 39

2.9.1. Subcellular localisation analysis by fluorescence microscopy............. 39

2.9.2. Submitochondrial localisation of proteins .......................................... 39

2.9.2.1. Preparation of mitoplasts ................................................ 40

2.9.2.2. Protease protection assay .............................................. 40

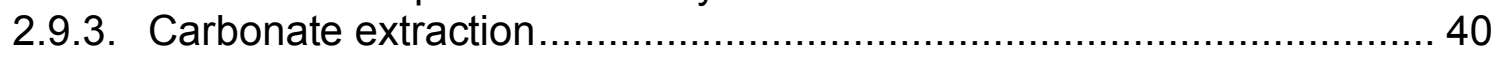

2.9.4. Protein precipitation with trichloroacteic acid (TCA) ....................... 41

2.10. Purification of mitochondrial protein complexes.................................. 41

2.10.1. Protein copurification via IgG chromatography............................... 41

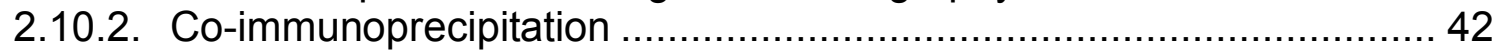

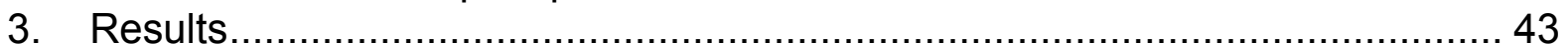

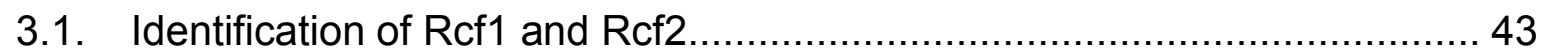

3.1.1. Purification and analyses of respiratory chain supercomplexes .......... 43 
3.1.2. Assaying precursor import of candidate proteins .............................. 45

3.1.3. Localization and topology of Rcf1, Rcf2 and Mio10 …...................... 47

3.1.4. Rcf1 and Rcf2 associate to respiratory chain supercomplexes ........... 49

3.1.5. Rcf1 and Rcf2 are associated with the cytochrome oxidase ............... 51

3.1.6. Rcf1 is conserved among eukaryotes ........................................... 52

3.1.7. Localization and topology of RCF1a and RCF1b ............................ 54

3.1.8. Human RCF1a and RCF1b proteins are complex IV components ....... 56

3.2. rcf1 $\Delta$ cells are growth deficient when grown on non-fermentable carbon

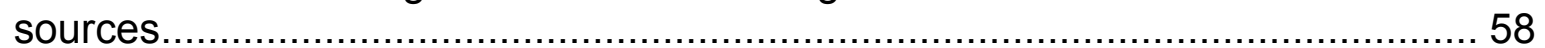

3.3. Cox13 steady state protein levels are reduced in $\operatorname{rcf} 1 \Delta \ldots \ldots \ldots \ldots \ldots \ldots \ldots \ldots \ldots . . . .59$

3.4. Loss of Rcf1/Cox13 affects overall oxygen consumption in mitochondria .. 60

3.4.1. Establishment of oxygen consumption assays................................ 60

3.4.2. $r c f 1 \Delta$ and $\operatorname{cox} 13 \Delta$ mitochondria displayed reduced oxygen consumption rates 61

3.5. In the absence of Rcf1/Cox13, cytochrome oxidase activity is altered ....... 62

3.5.1. Establishment of an enzyme activity assays .................................... 62

3.5.2. $r c f 1 \Delta$ displays reduced cytochrome oxidase activity while both mutants,

$r c f 1 \Delta$ and $r c f 2 \Delta$, have reduced aconitase activity ........................................ 63

3.6. Mitochondria isolated from $\operatorname{rcf} 1 \Delta$ and $\operatorname{rcf} 2 \Delta$ cells display increased ROS

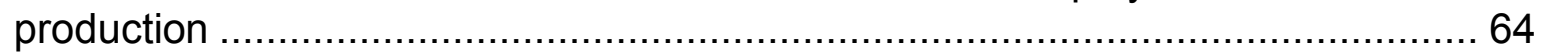

3.7. The RCF1 deletion phenotype is partially rescued by human RCF1b ........ 65

3.8. Assembly of Rcf2 and Cox13 is Rcf1 dependent .................................. 67

3.9. Formation of the supercomplexes in mitochondria requires Rcf1 .............. 70

3.10. Supercomplexes formed in mitochondria differ in regard to their

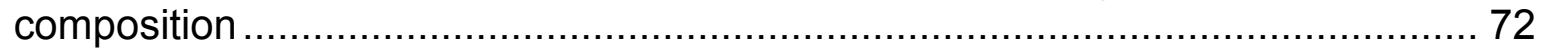

3.11. Distinct forms of cytochrome oxidase exist in mitochondria.................... 74

3.11.1. Purification of complex IV revealed its heterogeneous composition... 74

3.11.2. Rcf1 and Rcf2 can also interact with complex III .............................. 75

3.11.3. Not all complexes IV posses Cox13 ………................................. 76

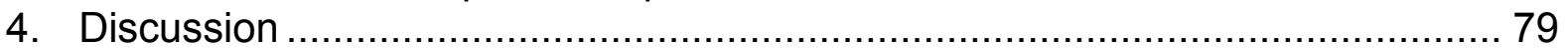

4.1. Identification of supercomplex interacting components …...................... 79

4.2. Rcf1 is a conserved component of complex IV ...................................... 80

4.3. Rcf1 requirement for complex IV activity is coupled to Cox13 function ........81

4.4. Rcf1 facilitates supercomplex formation in hypoxia.................................... 82

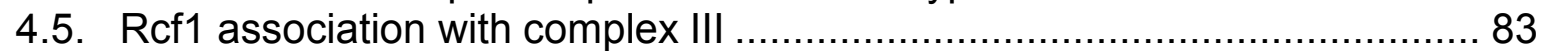

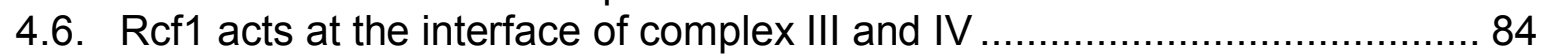

4.7. Implication of complex IV ${ }^{*}$ and Rcf1/2 in ROS production.......................... 89

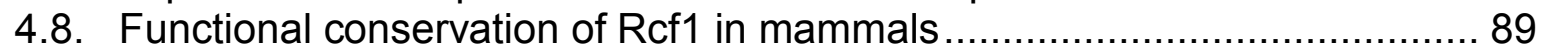

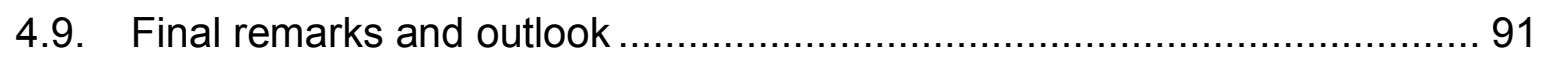

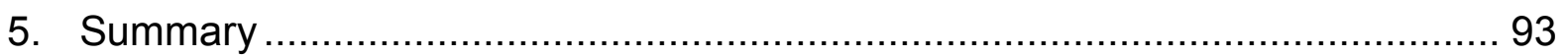

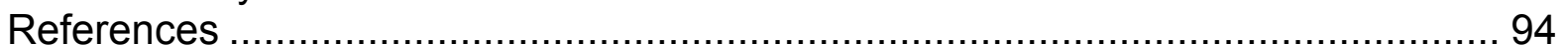

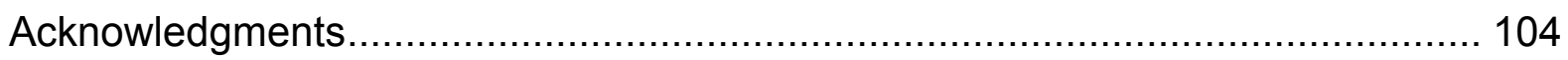

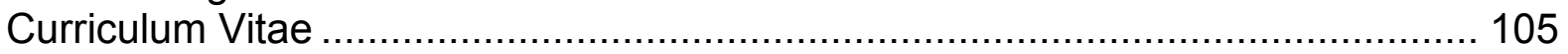




\section{Abbreviations}

AAC ADP/ATP carrier

ADP adenosine diphosphate

ATP adenosine triphosphate

BN-PAGE blue-native polyacrylamide gel electrophoresis

BSA bovine serum albumine

DNA deoxyribonucleic acid

EDTA ethylenediaminetetraacetic acid

$\mathrm{FADH}_{2}$ flavin adenine dinucleotide

HEPES 4-(2-hydroxyethyl)-1-piperazineethanesulfonic acid

$\mathrm{H}_{2}$ DCFDA $\quad 2$ ',7'-dichlorodihydrofluorescein diacetate

Ig $\quad$ immunoglobulin $\mathrm{G}$

IMS intermembrane space

MOPS 3-(N-morpholino)propanesulfonic acid

$\mathrm{NADH}$ nicoticamide adenine dinucleotide

OD optical density

PAM presequence translocase associated motor

PCR poymerase chain reaction

PFA paraformaldehyde

PMSF phenylmethylsulfonyl fluoride

PVDF poyvinylidene fluoride

RNA ribonucleic acid

ROS reactive oxygen species

RT-PCR real time poymerase chain reaction

SDS-PAGE sodium dodecyl sulfate polyacrylamide gel electrophoresis

TCA tricarboxylic acid

TIM translocase of the inner membrane

TM transmembrane

TOM translocase of the outer membrane

Tris tris(hydroximethyl)-aminomethan

$\Delta \psi \quad$ membrane potential 


\section{List of figures}

Figure 1. 1 Oxidative phosphorylation system in mitochondria........................... 2

Figure 1. 2 Biogensis of oxidative phosphorylation proteins. .............................. 7

Figure 1. 3 Schematic representation of electron flux in two models ...................... 11

Figure 1. 4 Models of the respiratory chain supercomplexes ............................. 12

Figure 3. 1Purification of Respiratory Chain Supercomplexes............................. 44

Figure 3. 2 Import of candidate proteins depends on membrane potential............ 47

Figure 3. 3 Rcf1, Rcf2 and Mio10 are membrane proteins.. .............................. 48

Figure 3. 4 Rcf1, Rcf2 and Mio10 are mitochondrial inner membrane proteins...... 49

Figure 3. 5 Rcf1 and Rcf2 associate with respiratory chain supercomplexes......... 50

Figure 3. 6 Rcf1 and Rcf2 co-isolate with purified respiratory chain supercomplexes.

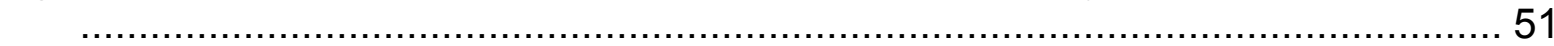

Figure 3. 7 Rcf1 and Rcf2 associate to cytochrome oxidase. ............................ 52

Figure 3. 8 Rcf1 is conserved among eukaryotes, while Rcf2 is conserved among

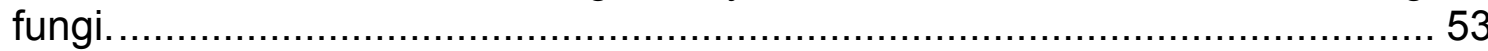

Figure 3. 9 Expression profiles of RCF1a and RCF1b in different mouse tissues. .. 54

Figure 3. 10 RCF1a and RCF1b, are localised within mitochondria.. .................... 55

Figure 3. 11 RCF1a and RCF1b, are localised to the inner membrane of

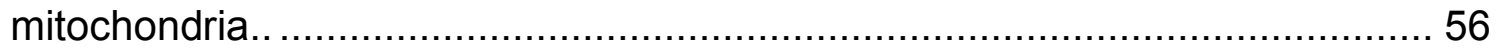

Figure 3. 12 RCF1a and RCF1b interact with cytochrome oxidase. .................... 57

Figure 3. 13 Growth analyses of $r c f 1 \Delta$ and $r c f 2 \Delta$ cells.................................... 59

Figure 3. 14 Steady state protein levels analysis of WT, $r c f 1 \Delta, r c f 2 \Delta$ and $\operatorname{cox} 13 \Delta$ mitochondria. ................................................................................... 60

Figure 3. 15 Oxygen consumption in $r c f 1 \Delta$ mitochondria is reduced. ................... 61

Figure 3. 16 Enzyme activities of mitochondria isolated from WT, coa3 $\Delta$ and $\operatorname{cox} 14 \Delta$

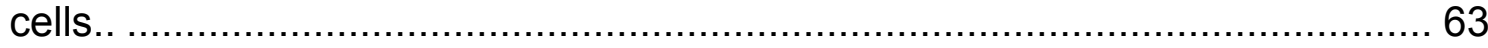

Figure 3. 17 Enzyme activities of WT, $r c f 1 \Delta, r c f 2 \Delta$ and $\operatorname{cox} 13 \Delta$ mitochondria. ........ 64

Figure 3. $18 r c f 1 \Delta$ and $r c f 2 \Delta$ mitochondria exert higher ROS production................. 65

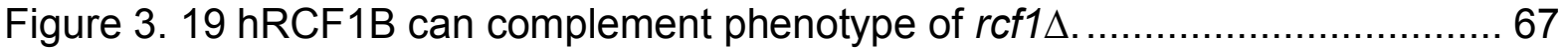

Figure 3. 20 Assemblies of Cox13 and Rcf2 into cytochrome c oxidase are Rcf1-

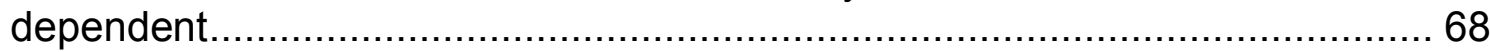

Figure 3. 21 Supercomplex formation depends on Rcf1 ............................... 71

Figure 3. 22 Isolation of supercomplexes via Cox $4^{\mathrm{ZZ}}$, Cox $13^{\mathrm{ZZ}}$ and Rcf2 ${ }^{\mathrm{ZZ}}$ proteins.73

Figure 3. 23 Steady state analysis of mitochondria overexpressing Rcf $2 \ldots \ldots \ldots \ldots \ldots .74$

Figure 3. 24 Purification of Cox $4^{\mathrm{ZZ}}$, Cox $13^{\mathrm{ZZ}}$ and Cor $1^{\mathrm{ZZ}}$ containing complexes..... 75

Figure 3. 25 Complex IV exists in distinct forms .............................................. 77

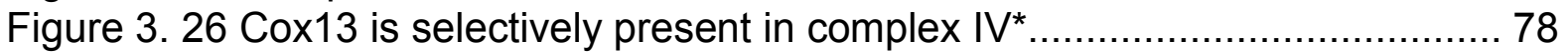

Figure 4. 1 Rcf1 facilitates interaction of complex $\mathrm{III}_{2}$ and IV ............................ 84

Figure 4. 2 Model for complex IV assembly.................................................. 85

Figure 4. 3 Model for the assembly of mitochondrial respiratory supercomplexes... 87 


\section{List of tables}

Table 2.1 Special chemicals and consumables.......................................

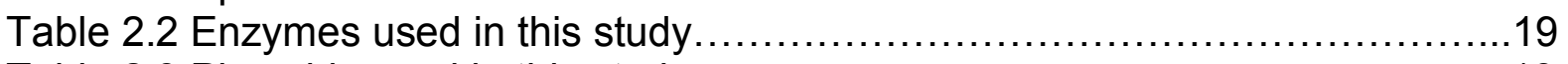

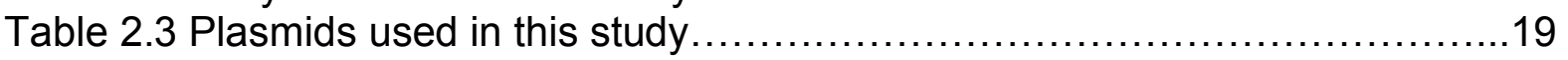

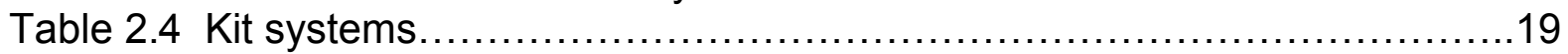

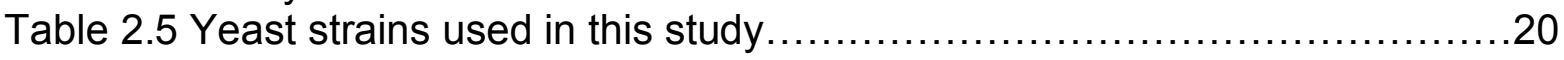

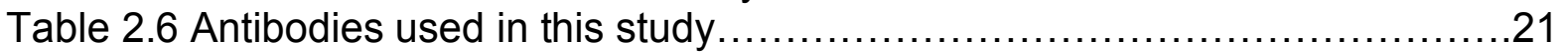

Table 3.1 Proteins of respiratory chain supercomplexes found by

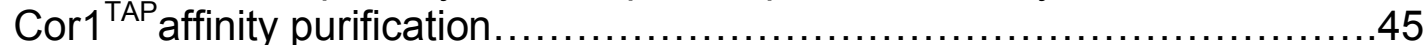

Table 3.2 Prediction of mitochondrial targeting for candidate proteins $\ldots \ldots \ldots \ldots \ldots . . . .46$ 



\section{Introduction}

\subsection{Mitochondria}

Mitochondria are ubiquitous organelles of eukaryotic cells. These organelles represent remnants of a prokaryotic organism, an $\alpha$-proteobacterium, which become a partner in a symbiotic relationship with the host cell over 1.5 billion years ago (Gray et al., 2001; Dyall et al., 2004). The interaction between the endosymbiont and the host cell resulted in the transfer of the endosymbiont's genes to the nucleus (Wallace, 2007) and the subsequent loss of redundant genes. Thus, the mitochondrial genome retained only a small subset of genes, which encode for only a few mitochondrial proteins (thirteen in humans and eight in yeast, Saccharomyces cerevisiae). The rest of the mitochondrial proteins are nuclear-coded, synthesized on cytosolic ribosomes and imported into mitochondria (Chacinska et al., 2009; Schmidt et al., 2010). Proteins coded by the mitochondrial genome are mainly hydrophobic components of the respiratory chain (Wallace, 2007). Therefore, mitochondria possess a machinery that facilitates maintenance and expression of its genome. Around $25 \%$ of the mitochondrial proteome participates in these events (Sickmann et al., 2003).

The most prominent function of mitochondria is the generation of adenosine triphosphate (ATP) through oxidative phosphorylation. Besides this, they are responsible for many other functions such as the metabolism of amino acids, $\beta$ oxidation of fatty acids, tricarboxylic acid cycle (TCA), generation of iron-sulfur clusters and calcium signalling (Lill and Muhlenhoff, 2005; Graier et al., 2007). Moreover, they play a crucial role in the generation/detoxification of reactive oxygen species (ROS), aging, lifespan regulation and apoptosis (Scorrano 2009; Finkel 2011; Pan, 2011). As mitochondria are involved in so many cellular processes, their dysfunction leads to diverse human disorders and diseases (Wallace, 2005; Chan, 2006; DiMauro and Schon, 2008). 


\subsection{Oxidative phosphorylation and the electron transport chain}

A central function of mitochondria is energy generation through oxidative phosphorylation. In mitochondria, $\mathrm{NADH}$ and $\mathrm{FADH}_{2}$ donate their electrons, derived from catabolic reactions, to the respiratory chain, transferring them to molecular oxygen and ultimately reducing it to water (Figure 1.1A). Electron transport is coupled to the translocation of protons from the matrix into the intermembrane space (IMS), which results in a charge gradient and thus in a $\mathrm{pH}$ gradient across the inner mitochondrial membrane. These gradients provide the energy (proton-motive force) for ATP synthesis from ADP and $P_{i}$ via the $F_{1} F_{0}$-ATP-Synthase (Mitchell, 1968; Saraste, 1999).
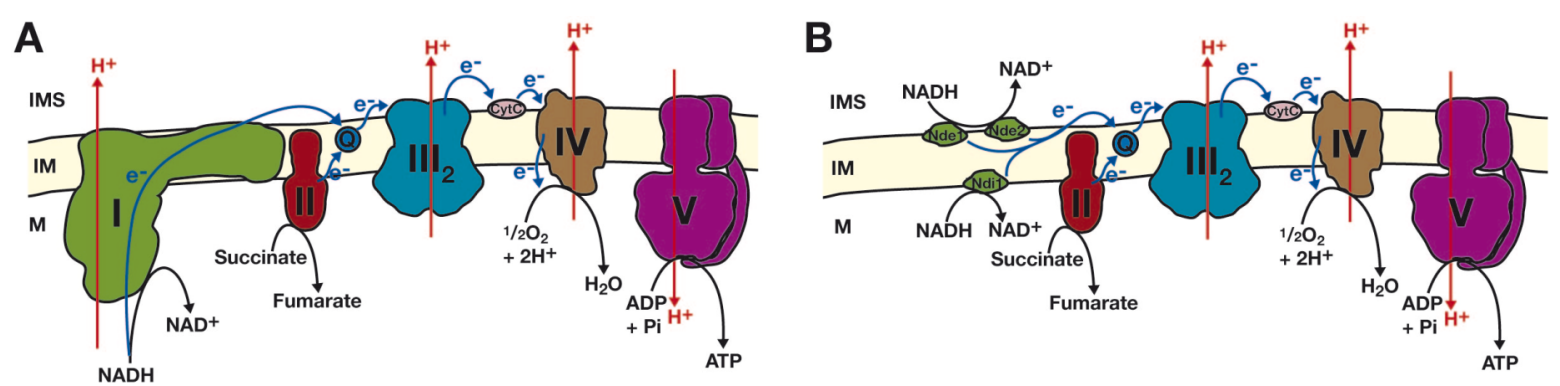

Figure 1. 1 Oxidative phosphorylation system in mitochondria. (A) Shown are the respiratory chain complexes (I-IV) and the $F_{1} F_{0}$-ATP-Synthase $(V)$ in the inner mitochondrial membrane (IM) of mammalian mitochondria. (B) Respiratory chain complexes (II-IV) and $F_{1} F_{0}$-ATP-Synthase $(\mathrm{V})$ in the IM of yeast mitochondria. Electron flux $\left(\mathrm{e}^{-}\right)$along the respiratory chain is depicted by blue arrows. Red arrows $\left(\mathrm{H}^{+}\right)$indicate proton flux across the IM. Q, coenzyme Q; CytC, cytochrome $c$; IMS, intermembrane space; M, matrix.

The mitochondrial respiratory chain consists of four electron-transporting complexes, designated as the NADH-ubiquinone oxidoreductase (complex I), succinate dehydrogenase (complex II), ubiquinol-cytochrome $c$ reductase (complex III) and cytochrome oxidase (complex IV) (Saraste, 1999), and the $F_{1} F_{0}$-ATP-Synthase (complex V). These are multimeric enzymes, which are embedded in the inner mitochondrial membrane and, with the exception of complex II, are composed of subunits encoded by both the mitochondrial and nuclear genomes. Complexes I, III 
and IV serve as proton pumps. Unlike most eukaryotes, the respiratory chain of yeast S. cerevisiae lacks a multisubunit complex I (Figure 1.1B). Instead, it contains three different NADH dehydrogenases; Nde1 and Nde2, facing the IMS side of inner membrane and Ndi1, facing the matrix side of the inner membrane (GrandierVazeille et al., 2001). Thus, only complexes III and IV are responsible for proton pumping in S. cerevisiae mitochondria. Electron transfer between the complexes is achieved by two mobile redox-active molecules; ubiquinone (coenzyme $Q$ ) and cytochrome $c$. Ubiquinone is a free diffusible electron carrier within the inner mitochondrial membrane which transports pairs of electrons from complexes I and II to complex III, while cytochrome $c$ is located on the external surface of the inner membrane and transfers electrons from complex III to complex IV (Figure 1). Electron shuttling within individual complexes is facilitated by different redox-centers; iron-sulfur clusters, cytochromes and $\mathrm{Cu}^{2+}$.

Structure and function of respiratory complexes has long been a subject of intense research. In fact, most of the respiratory chain complexes have been isolated form various organisms and their structures have been resolved at the atomic level (Tsukihara et al., 1996; Hunte et al., 2000; Hunte et al., 2010).

\subsection{Ubiquinol-cytochrome c reductase}

Ubiquinol-cytochrome $c$ reductase or $b c_{1}$ complex (complex III) is a multi-subunit enzyme of the respiratory chain that catalyze electron transfer from ubiquinol to cytochrome c. Complex III operates through a Q-cycle mechanism that couples electron transfer to proton translocation across the membrane (Trumpower et al., 1994; Crofts, 2004). It is present in all eukaryotes, as well as in many aerobic and photosynthetic bacteria. High-resolution structures have shown that the $b c_{1}$ complex exists as a symmetrical homodimer in eukaryotes (Xia et al., 1997; Iwata et al, 1998; Hunte et al., 2000; Lange et al., 2002).

The catalytic core of the complex contains three essential protein subunits with redox prosthetic groups; cytochrome $b$ with two $b$-type hemes (high-potential heme $b_{H}$ and a lower potential heme $b_{\mathrm{L}}$ ), cytochrome $c_{1}$ and an iron-sulfur protein with $2 \mathrm{Fe}-2 \mathrm{~S}$ clusters. These subunits exhibit high evolutionary conservation and participate in 
electron transfer and proton translocation activities of the enzyme (Trumpower, 1990; Smith et al., 2004). In addition to these, mitochondrial complex III also possesses nonredox subunits; termed as supernumerary subunits, the function of which is not known and are most possibly involved in the structural stability of the complex. In yeast, S. cerevisiae, these subunits consist of two core proteins, Cor1 and Cor2, and five additional subunits; Qcr6, Qcr7, Qcr8, Qcr9 and Qcr10. The mammalian enzyme contains one additional subunit, corresponding to the cleaved $\mathrm{N}$-terminal sequence of Rip1 (Brandt, 1993). With the exception of cytochrome $b$, encoded by mitochondrial DNA, all $b c_{1}$ components are of nuclear origin.

\subsection{Cytochrome oxidase}

The terminal enzyme of the mitochondrial respiratory chain is cytochrome oxidase. The complex is comprised of 13 subunits in human and 11 subunits in yeast, $S$. cerevisiae, which have dual genetic origin (Tsukihara et al., 1996; Yoshikawa et al., 1998). The three largest subunits, Cox 1, Cox 2 and Cox 3 , are encoded by mitochondrial DNA and exhibit high conservation among all respiring organisms (Castresana et al., 1994). These subunits form the catalytic core of the enzyme. The remaining subunits are nuclear-coded and are present only in eukaryotes.

The enzyme's catalytic core contains four sites for redox active metals; $\mathrm{Cu}_{\mathrm{A}}$, located in Cox2 subunit and heme $a$, heme $a_{3}$ and $\mathrm{Cu}_{\mathrm{B}}$, located in Cox1 (Tsukihara et al., 1995). Electrons from reduced cytochrome $c$ are transferred to the binuclear center of $\mathrm{Cu}_{\mathrm{A}}$ and subsequently shuttled to heme a, followed by transition to oxygen, located in the oxygen reduction site formed by heme $\mathrm{a}_{3}$ and $\mathrm{Cu}_{\mathrm{B}}$. This process is coupled to proton translocation from the matrix into the intermembrane space (Belevich et al., 2006; Yoshikawa et al., 2011).

The exact function of nuclear-coded subunits within the complex is not yet clear, but it is known that they are necessary for the assembly, stability and function of complex IV ( $\mathrm{Li}$ et al., 2006; Galati et al., 2009). Additionally, certain subunits are found to exhibit tissue-specific isoformes, such as the mammalian COX6a (homolog of yeast Cox13), possessing two isoforms; the heart isoform expressed in contractile muscle and liver isoform expressed in most tissues (Mell et al., 1994). 


\subsubsection{Composition of the cytochrome oxidase is oxygen-dependent}

During a cells' life span it experiences various oxygen tensions, thus it is of great importance for survival to sense and respond to changes in environmental oxygen availability. By regulating levels and activities of various proteins, cells adjust their metabolic pathways in order to adapt to different oxygen concentrations (Poyton, 1999). The effect of oxygen on many of these proteins is exerted at the level of transcription, but in some occasions oxygen's effect takes place at the posttranscriptional level (Burke et al., 1997; Semenza, 2007; Tello et al., 2011).

The respiratory chain is one of the systems within the cell where function could be modulated in response to oxygen availability by generation of cytochrome oxidase isozymes with altered activities (Waterland et al., 1991; Allen et al., 1995; Fukuda et al., 2007). This is achieved through differential expression of homologues subunits isoforms (Cox5a and Cox5b in yeast, and COX4-1 and COX4-2 in mammals) whose genes are inversely regulated by oxygen (Fukuda et al., 2007; Burke and Poyton, 1998). Two isoforms affect the catalytic properties of cytochrome oxidase by altering an internal step in electron transfer between heme and the binuclear reaction center (Waterland et al., 1991; Allen et al., 1995). Although yeast Cox5 and mammalian COX4 have remarkably similar functions and their expression is regulated by oxygen, the underlying molecular mechanisms are quite different. The manner by which cells sense oxygen and the steps involved in signal transduction have not been precisely defined. However, it has been reported that in yeast, heme could play a role in monitoring oxidative stress (Zytomer and Lowry, 1992; Bunn and Poyton, 1996). In aerobic conditions, yeast cells are able to synthesize heme and the activation of transcription factors Hap2/3/4/5 and Hap1 occurs. Hap2/3/4/5 activates the transcription of COX5a and Hap1 activates the transcription of ROX1, which represses the transcription of COX5b (Kwast et al., 1998). During hypoxic conditions when heme biosynthesis is compromised, Hap2/3/4/5 and Hap1 are not active and thus the transcription of COX5a is abolished, while transcription of COX5b is derepressed (Burke and Poyton, 1998). In mammalian cells, the regulation of COX41 and COX4-2 depends on HIF-1 (hypoxia inducible factor 1), which activity is 
regulated by oxygen-dependent hydroxylation (Ivan et al., 2001; Yu et al., 2001). Under aerobic conditions, HIF-1 is hydroxylated and continuously proteasomally degraded. In hypoxia, hydroxylation is inhibited and HIF-1 is stabilized, which stimulates the expression of the COX4I2 gene, which encodes COX4-2. Also, the transcription of the LON gene, which encodes the LON protease necessary for COX4-1 degradation, is stimulated by HIF-1. Thus, during hypoxia, synthesis of the COX4-2 protein and the proteolysis of COX4-1 are increased (Fukuda et al., 2007).

\subsubsection{Biogenesis of cytochrome oxidase}

To generate mature complex IV, both mitochondrial- and nuclear-encoded subunits must assemble together in addition to the insertion of co-factors. Mitochondrialencoded subunits have to be inserted into the inner mitochondrial membrane from the matrix side, while nuclear-encoded subunits have to be imported into mitochondria.

\subsubsection{Import of mitochondrial proteins}

Mitochondrial proteins encoded by the nuclear genome are synthesized on cytosolic ribosomes as precursor proteins and imported into the organelle (Becker et al., 2012). Precursor proteins are present in the cytosol in an unfolded conformation, stabilized via their association with chaperones, mostly Hsp70, which prevent their aggregation and degradation (Young et al., 2003). Proteins synthesized in the cytosol possess targeting signals that direct them to mitochondria, where they are recognized by the receptors exposed on the organelle surface, and also promote protein sorting to the correct mitochondrial compartment.

Most of the respiratory chain proteins contain a cleavable $\mathrm{N}$-terminal targeting signal, termed a presequence. The import route of presequence-containing preproteins involves two translocases: translocase of the outer membrane (TOM complex) and the presequence translocase of the inner membrane (TIM23 complex). 


\subsection{The translocase of the outer mitochondrial membrane}

The TOM complex represents the main entrance for almost all nuclear-encoded mitochondrial proteins (Figure 1.4) (Endo and Yamano, 2010). It is comprised of Tom40, an integral membrane protein that forms the central pore for translocation of proteins across the outer membrane (Hill et al., 1998; Ahting et al., 2001; Becker et al., 2005), three receptor proteins, Tom20, Tom22 and Tom70, and additional small TOM proteins. After initial recognition and binding of the preproteins, mediated by either Tom20 or Tom70 (Brix et al., 1997), preproteins are handed over to Tom22, and then with the help of Tom5 they are inserted into the Tom40 pore. On the intermembrane space side of the outer membrane, Tom22 exposes a C-terminal domain that binds the translocating preprotein.

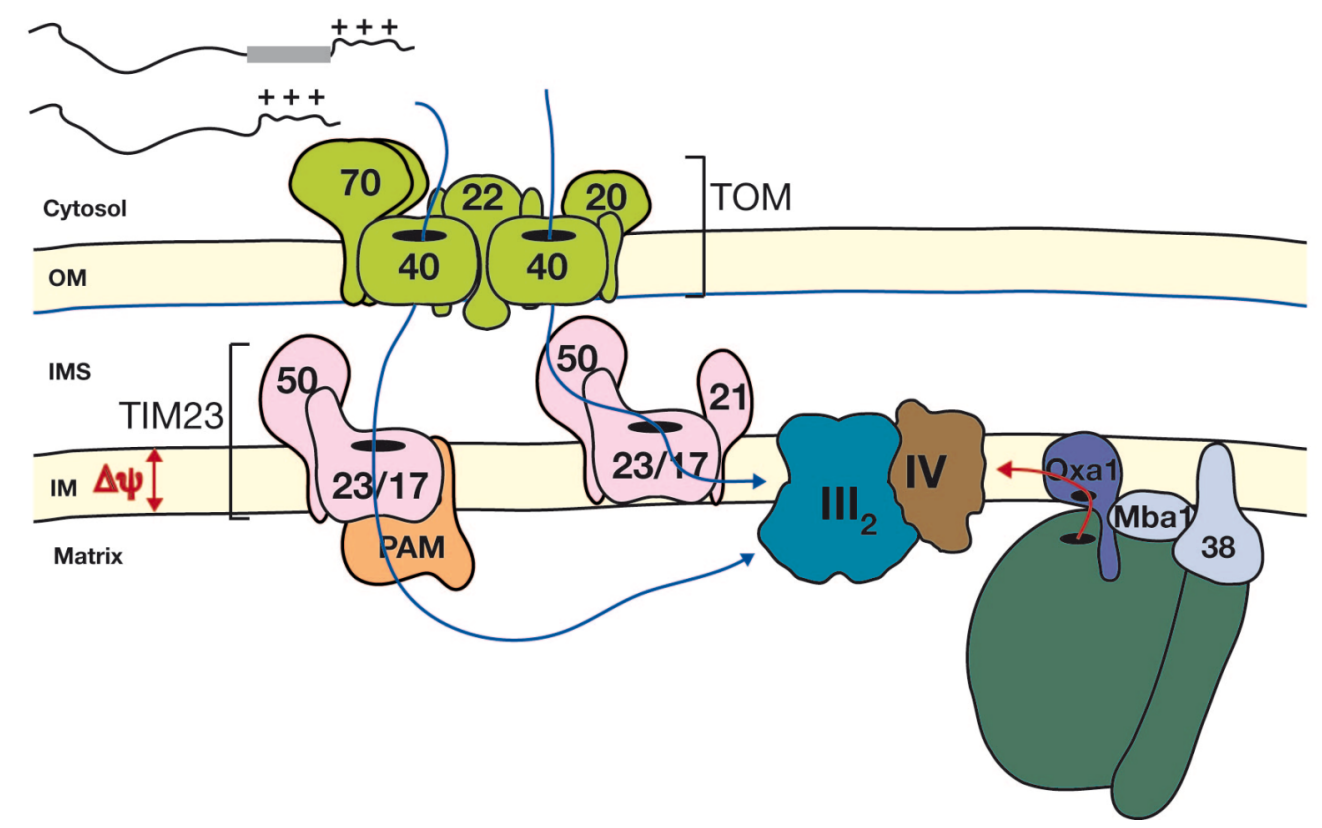

Figure 1. 2 Biogensis of oxidative phosphorylation proteins. Precursor proteins synthesized in the cytosol take one of two import routes (blue arrows). Both pass the other mitochondrial membrane (OM) through the translocase of the outer membrane (TOM, green). Precursors are further translocated into or across the inner mitochondrial membrane (IM), with a help of presequence translocase of the inner mitochondrial membrane (TIM23, pink). Depending on the precursor TIM23 can either associate with the presequence translocase-associated motor (PAM, orange) or the Tim21 protein (21). Mitochondrial-encoded proteins follow mitochondrial export pathway (red arrow). See the text for the details. IMS, intermembrane space, $\Delta \psi$, membrane potential across the inner membrane. Numbers indicate the protein subunits of the respective complexes. 


\subsection{The presequence translocase of the inner mitochondrial membrane}

After passing the outer mitochondrial membrane, presequence containing preproteins are handed over from the TOM to the TIM23 complex, at which point they are directed either to the inner membrane or to the mitochondrial matrix (Figure 1.2). The core of the TIM23 complex is composed of three essential transmembrane proteins: Tim23, which forms the pore of the presequence translocase (Truscott et al., 2001), Tim50 that binds presequences of preproteins emerging from the TOM complex and passes them to Tim23 (Mokranjac et al., 2009; Tamura et al., 2009; Schulz et al., 2011) as well as regulates the permeability of the TIM23 pore (Meinecke et al., 2006), and Tim17 involved in motor recruitment and lateral sorting of the preproteins (Chacinska et al., 2005). Core subunits associate with the fourth integral membrane protein, Tim21, that interacts with the intermembrane space domains of Tim23 and Tim50 with its own intermembrane space domain to assist in import via its transient interaction with the TIM23 complex (Chacinska et al., 2005; Mokranjac et al., 2009; Tamura et al., 2009). Tim21 also promotes transient coupling with respiratory complexes III and IV, allowing for efficient $\Delta \psi$-dependent translocation of preproteins (van der Laan et al., 2006; Wiedemann et al., 2007).

For preprotein integration into the inner membrane, the TIM23 complex composed of these four components is sufficient, and this form of the complex is referred to as TIM23 ${ }^{\text {SORT }}$ (van der Laan et al., 2007). Preproteins that follow this insertion pathway contain a hydrophobic sorting signal behind the presequence, which arrests them in the inner membrane and causes their lateral release into the inner membrane (Glick et al., 1992).

If the preproteins are destined for the matrix, they require complete translocation across the inner mitochondrial membrane, which is preformed by an ATP-dependent process mediated by the PAM (presequence translocase associated motor) machinery, which associates to the TIM23 complex lacking Tim21. This form of the presequence translocase is designated as the TIM23 ${ }^{\text {MOTOR }}$ (Chacinska et al., 2010). Upon arrival of the preprotein in the matrix, the presequence is proteolytically 
cleaved by the mitochondrial processing peptidase (MPP), at which point the mature protein is released (Luciano and Geli, 1996).

\subsubsection{Export of mitochondrial-encoded proteins}

In contrast to nuclear-encoded mitochondrial proteins, the insertion of mitochondrialencoded proteins in the inner mitochondrial membrane is co-translational, and depends on the mitochondrial export machinery (Ott and Hermann, 2010). The Oxa1 insertase is the central component of co-translational insertion machinery (Altamura et al., 1996; Hell et al., 2001). Oxa1 is able to bind mitochondrial ribosomes through its C-terminus, which facilitates co-translational insertion of nascent polypeptides (Szyrach et al., 2003; Jia et al. 2003). Additional factors such as Mba1 and Mdm38 are required for this process (Preuss et al., 2001; Ott et al. 2006; Frazier et al., 2006).

\subsubsection{Assembly of cytochrome oxidase}

Assembly of cytochrome oxidase requires participation of diverse assembly factors, the function of which is largely unresolved. They are involved in all steps of complex formation, including transcription and translation of mitochondrial-coded subunits (Mick, 2011; Soto, 2011), membrane insertion of the core subunits (Hell, 2001), heme a biosynthesis (Barros et al., 2001; Barros and Tzagoloff; 2002) and copper and heme $a_{3}$ insertion (Cobine, 2006; Khalimonchuk et al., 2010).

The translation of mitochondrial-encoded proteins is tightly linked to their assembly, with Cox1 being one of the most characterized examples. This coupling allows for negative feedback regulation of mitochondrial translation with respect to the cytochrome oxidase assembly status. The translational activators, Mss51 and Pet309, are essential for translation of Cox1 (Decoster et al., 1990; Manthey et al., 1995; Perez-Martinez et al., 2003). Mss51 is able to dynamically interact with Cox1 and several assembly factors of cytochrome oxidase (Barrientos et al., 2004; PerezMartinez et al., 2009). During synthesis of Cox1, Mss51 and newly synthesized Cox1 interact and form a transient complex, stabilized by assembly factors Cox14 and Coa3 (Barrientos et al., 2004; Mick et al., 2010). In this complex Mss51 is trapped 
and unable to promote Cox1 expression. An additional assembly factor, Coa1, also participates in sequestration of Mss51 (Pierrel et al., 2007; Mick et al., 2007). Release of Mss51 from this complex leads to its reactivation and initiation of Cox1 translation, although it is not clear what is the mechanism that promotes this event. Translation, membrane insertion and maturation of Cox1 represent the first steps in cytochrome oxidase biogenesis, followed by the sequential incorporation of structural subunits, leading to the formation of mature complex IV (Nijtmans et al., 1998).

\subsection{Respiratory chain supercomplexes}

For a long time it was believed that respiratory chain complexes are independent entities that freely diffuse within the inner mitochondrial membrane and that the electron transfer between them is supported by their random collision (Hackenbrock et al., 1986). This model, the "fluid state" model (Figure 1.3A), was widely accepted with the main argument being the isolation of individual respiratory complexes in their active form. Accumulating data in the last decade evidenced the existence of a higher organisation level of respiratory complexes and pointed toward a different model, the "solid state" model (Figure 1.3B). In fact, individual complexes can interact and associate into so called respirasomes, supercomplex structures composed of several respiratory complexes. Although the interaction between individual complexes was suggested earlier, one of the first experimental findings for higher level respiratory chain complex organisation came from the discovery of supercomplexes in yeast and bovine heart mitochondria resolved by Blue-native PAGE (BN-PAGE) (Schägger and Pfeiffer, 2000). Based on the BN-PAGE procedure, the existence of respirasomes was also reported for bacteria (Stroh et al., 2004), plant (Eubel et al., 2003) and human mitochondria (Schägger et al., 2004). Interaction of respiratory complexes was also confirmed by gel filtration and imunoprecipitation experiments (Cruciat et al., 2000). Additional evidence for supercomplex organisation was based on single particle electron microscopy 2D projection map analyses of these structures from different organisms, such as Arabidopsis (Dudkina et al., 2005), bovine (Schäfer et al., 2006) and yeast (Heinemeyer et al, 2007). Later, 3D projection maps of bovine mitochondria 
supercomplexes yielded more insights into relative positions and the interaction of complexes within these assemblies (Schäfer et al., 2007; Althoff et al., 2011; Dudkina et al., 2011).

A

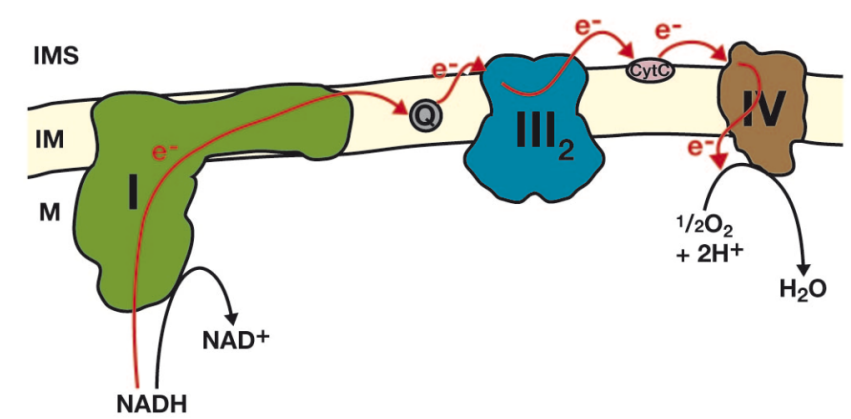

B

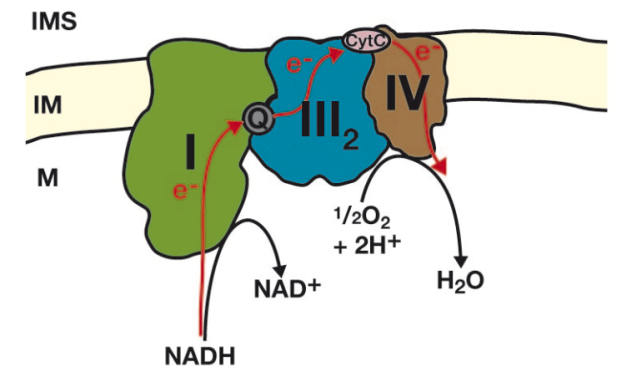

Figure 1. 3 Schematic representation of electron flux in two models. (A) Liquid model in which soluble electron carriers freely diffuse between complexes I, III and IV. (B) Solid model in which complexes I, III and IV are assembled in a supercomplex that trap electron carriers and thus restrict their diffusion. Q, coenzyme Q; CytC, cytochrome $c$; IMS, intermembrane space; M, matrix; IM, inner membrane.

\subsubsection{Diversity of respiratory supercomplexes}

The association of respiratory complexes into supercomplexes has been extensively investigated in a wide range of organisms. Although complexes I, III, IV and V were found to assemble into distinct oligomeric forms, supercomplexes containing complex II have yet to be discovered. The biggest respiratory enzyme, complex I, was found to be stably associated with a dimer of complex III $\left(\mathrm{I}_{1} \mid \mathrm{II}_{2}\right)$ in mitochondria of plants and mammals (Figure 1.4A) (Dudkina et al., 2005; Schägger and Pfeiffer, 2000). In addition to its binding to complex I, dimeric complex III was shown to interact with complex IV, making its oligomerization pattern even more intricate. In plant and bovine mitochondria supercomplexes comprising complex I, $\mathrm{III}_{2}$ and IV have been identified (Figure 1.4A). The number of complex IV copies that are present within these structures has been observed to be as high as four, and generated supercomplexes are designated as $\mathrm{I}_{1} \mid \mathrm{II}_{2} \mathrm{IV} \mathrm{V}_{1-4}$ (Eubel et al., 2004; 
Schägger, 2001). Due to the absence of complex I in S. cerevisiae, complex $\mathrm{III}_{2}$ can only associate to complex IV. It can bind with a single or two copies of complex IV, forming two different types of supercomplexes; $\mathrm{III}_{2} \mathrm{IV}$ and $\mathrm{III}_{2} \mathrm{IV} \mathrm{V}_{2}$ (Figure 1.4B) (Schägger and Pfeiffer, 2000; Heinemyer et al., 2007). Supercomplexes corresponding to $\mathrm{III}_{2} \mathrm{IV}$ were also identified in bovine and some plant mitochondria (Schägger and Pfeiffer, 2000; Dudkina et al., 2006). It was reported that complex V dimerizes in mitochondria of different eukaryotes from yeast to mammals (Arnold et al., 1998; Wittig et al., 2008). In addition to its appearance as a dimer, complex V can exist as a higher homo-oligomer in the mitochondrial inner membrane, such as tetramers (Krause et al., 2005; Thomas et al., 2008).

A

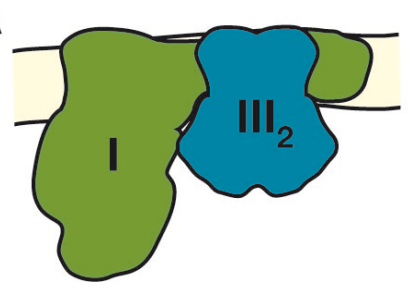

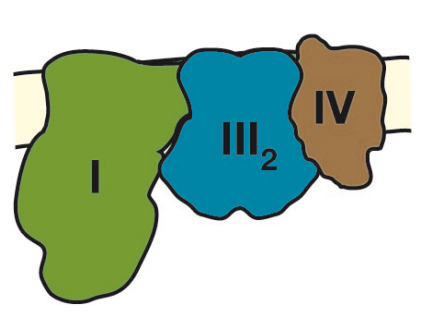

B

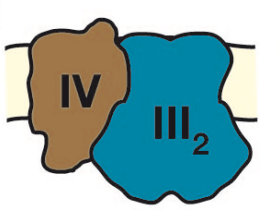

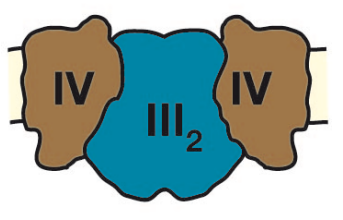

Figure 1. 4 Models of the respiratory chain supercomplexes. (A) $I_{1} I I_{2}$ and $I_{1} I I_{2} I V_{1}$ supercomplexes from mammalian mitochondria. (B) $\mathrm{II}_{2} \mathrm{IV}$ and $\mathrm{III}_{2} \mathrm{IV} \mathrm{V}_{2}$ supercomplexes from yeast, $S$. cerevisiae, mitochondria.

Although the existence of respiratory complexes has been verified by different approaches in various organisms, the co-existence of suprecomplexes with individual complexes was evident in all cases. Thus, the organization of the respiratory chain cannot be simply explained by two excluding models, but rather as a dynamic system. Recently, the so-called "plasticity" model has been introduced, which supports both models and suggests that respiratory complexes can switch between two forms; freely moving and supercomplex integrated, depending on the metabolic status of the cell (Acin-Perez et al., 2008). 


\subsubsection{Functional and structural significance of supercomplex formation}

The importance of supramolecular organization of respiratory system in mitochondria is not completely clear yet, but it might provide several structural and functional advantages. Such organization of respiratory complexes should improve their catalytic activity through the enhancement of electron flow and substrate channelling. A lot of evidences support the aforementioned hypothesis, obtained by investigating flux-control of electron transfer and kinetic properties of isolated supercomplexes (Boumans et al., 1998; Lenaz and Genova, 2010; Acin-Perez et al., 2008).

Association of respiratory complexes could be important for the assembly and stability of individual complexes. Several studies in mutants harbouring specific defects in single respiratory complexes have reported that complex III and IV are required for the maintenance of complex I (Acin-Perez et al., 2004; Diaz et al., 2006; $L i$ et al., 2007). This has important implications for human pathology, as various genetic etiologies of mitochondrial diseases primarily affecting the assembly of one complex could have a profound effect on others. For instance, mutations in mitochondria-encoded cytochrome $b$ that impair complex III formation could result in combined complex I and IV deficiencies in patients (Moran et al., 2010; Schägger et al. 2004).

Supercomplex formation could determine mitochondrial ultrastructure. In fact, oligomerization of complex $\mathrm{V}$ promotes membrane curvature and the formation of tubular cristae membrane (Velours et al., 2009; Paumard et al., 2002b). Dimerization of complex $\mathrm{V}$ is promoted by subunits $\mathrm{g}$ and $\mathrm{e}$ and in their absence mitochondria exhibit "onion-like" morphology without any membrane folding (Paumard et al., 2002b; Arselin et al., 2004).

In addition, it has been proposed that the organization of the respiratory chain in supermolecular structures could limit generation of reactive oxygen species (ROS), but currently no experimental confirmation has been obtained (Schägger, 2002). 


\subsubsection{Formation of respiratory supercomplexes}

Although the existence and implications of respiratory supercomplexes has been tested in a wide variety of organisms, the actual mechanism of their biosynthesis is still unsolved. Analyses of the time course of the incorporation of 13 mitochondriaencoded proteins into respiratory complexes and supercomplexes, by pulse-chase experiments, suggested that the formation of supercomplexes takes place upon the assembly of individual complexes through their direct interactions (Acin-Perez et al., 2008). In spite of this, it was revealed that the initial formation of individual complexes is not a prerequisite for supercomplex formation since nuclear-encoded complex IV components could bind directly to the supercomplexes in the absence of monomeric complex IV (Lazarou et al., 2009; Mick et al., 2007). Recent evaluation of supercomplex biosynthesis in human cells proposed their construction through multiple steps. It might start with a complex I intermediate that acts as a scaffold for sequential incorporation of complex III and IV subunits and subassemblies, and ends with the integration of the complex I catalytic core, which leads to the functional activation of the respirasome (Moreno-Lastres et al., 2012).

Complex $\mathrm{V}$ oligomerization has been largely characterized. It has been reported that this process specifically depends on several proteins (Wagner et al., 2009; Wagner et al., 2010). Still, no proteins facilitating interactions between complexes I, III and IV and thus propagating their oligomerization have been assigned. It has been revealed that the formation and stability of supercomplexes in the inner mitochondrial membrane relay on cardiolipin, phospholipid specific to the inner mitochondrial membane (Zhang et al., 2002; Zhang et al., 2005). In fact, in yeast cells in which cardiolipin biosynthesis is affected by the deletion of the CRD1 gene, encoding cardiolipin synthase, supercomplexes are destabilized (Pfeiffer et al., 2003). Supercomplex formation is compromised even in yeast cells affected in cardiolipin biosynthesis, exhibiting reduced amounts and altered acyl side chain, because of the lack of the TAZ1 gene responsible for cardiolipin remodelling (Brandner et al., 2005). Mutations in the gene encoding for the human ortholog of Taz1, tafazzin, are 
implicated in Barth syndrome, where affected patients exhibit a dissociation of supercomplexes (McKenzie et al., 2006).

\subsubsection{Interaction partners of respiratory supercomplexes}

Recent findings have pointed towards the notion that the supercomplex forms introduced above can interact with distinct proteins, leading to higher organizational complexity of these structures. It has been observed that the TIM23 protein translocase and its associated import motor, the PAM machinery, can interact with respiratory chain complexes (van der Laan et al., 2006; Wiedemann et al., 2007; Saddar et al., 2008). Also, respiratory chain assembly factors and translational regulators were found in association with supercomplexes (Mick et al., 2007; Mick et al., 2010). In addition, the major yeast ADP/ATP carrier, AAC, has also been shown to physically interact with respiratory supercomplexes (Dienhart and Stuart, 2008). In each case, only subpopulations of supercomplexes were identified to interact with the aforementioned proteins. Thus, the interaction of supercomplexes with different protein groups gives rise to their diversity within mitochondria. The functional relevance of these associations is not completely clear, but it has been shown that AAC could maintain the formation of the $\mathrm{II}_{2} \mathrm{IV}_{2}$ form in yeast (Dienhart and Stuart, 2008). Also, it has been proposed that the possible function of the interaction between respiratory complexes and the TIM23 translocase and PAM machinery could be to support membrane potential $(\Delta \psi)$ dependent protein import (van der Laan et al., 2006; Wiedemann et al., 2007). 


\subsection{Research objectives}

The complexes of the electron transport chain interact with each other allowing for the assembly of higher-order structures, known as respirasomes or supercomplexes. Supercomplex formation is important for the stabilization of individual complexes, efficient electron transfer and sequestration of ROS. Although, the existence of supercomplexes has been shown in a variety of organisms, their formation is poorly understood. Cardiolipin is important for supercomplex maintance, but the existence of additional specific factors that facilitate the assembly and stability of these structures is enigmatic.

The aim of this work was to purify respiratory supercomplexes from isolated yeast mitochondria and to analyse their composition by mass spectrometry. This analyses uncovered several uncharacterised proteins observed to copurify with supercomplexes. The specificity of their isolation, as well as verification of mitochondrial localization and the association of these proteins with supercomplexes was assessed by employing different biochemical approaches. Furthermore, this study aims to elucidate the function of proteins specifically isolated with supercomplexes. For this purpose, deletion mutants of the corresponding genes were generated and subjected to detailed analyses. Thus, the main focus of this work was to assess if any of the newly identified proteins might play a role in supercomplex formation. 


\section{Materials and Methods}

\subsection{Materials}

\subsubsection{Chemicals}

Standard chemicals were of analytical grade and purchased from AppliChem (Darmstadt), Merck (Darmstadt), Sigma Aldrich (Taufkirchen), Roth (Karlsruhe) or Serva (Heidelberg). Chemicals listed in table 2.1 were used in this study.

\subsubsection{Enzymes, plasimds and kits}

Restriction enzymes for cloning were obtained from Fermentas (St. Leon-Rot). Deoxyoligonucleotides were purchased in standard desalted quality from Metabion (Martinsried) and used as primers for PCR. All restriction enzymes, ligases and polymerases were used according to supplier's instructions (including the supplied buffers). Special enzymes listed in table 2.2 were used in this study. Plasmids listed in table 2.3 were used in this study. Plasmid generation was performed as in section 2.5.6. The commercially available kit systems that were used in this study are listed in table 2.4 and were used for the stated application according to manufacturer's instructions.

\subsubsection{Microorganisms and cell lines}

For molecular cloning, the Escherichia coli (E. coli) strain XL1-blue (Stratagene) was used. Saccharomyces cerevisiae (S. cerevisiae) strains used in this study are listed in table 2.5. For human cell culture, HEK-293T cells (ATCC, CRL-11268), adherent, immortalized human embryonic kidney cells were used. For immunofluorescence assays U2-OS, adherent, immortalized osteoblast cells were used.

\subsubsection{Antibodies}

Polyclonal antisera directed against yeast and human proteins were raised in rabbit, immunized with peptides (Gramsch Laboratories, Schwabhausen) unless otherwise stated. Secondary antibodies coupled to horseradish peroxidase against rabbit lgG, 
were used for Western blot detection. Antibodies used in this study are listed in table 2.6 .

Table 2.1 Special chemicals and consumables

\begin{tabular}{|c|c|}
\hline Product & Manufacturer \\
\hline ATP & Roche \\
\hline $\mathrm{NADH}$ & Roche \\
\hline Creatine phosphate & Roche \\
\hline$\left[{ }^{35}\right.$ S $]$ methionine & Hartmann-Analytic \\
\hline SDS-Marker: Broad Range & Biorad \\
\hline BN-Marker: HMW calibration kit & GE-Healthcare \\
\hline DNA-Marker: GeneRuler & Fermentas \\
\hline Digitonin & Calbiochem \\
\hline Triton $\mathrm{X}-100$ & Serva \\
\hline PMSF & Sigma Aldrich \\
\hline AntimycinA & Sigma Aldrich \\
\hline Oligomycin & Sigma Aldrich \\
\hline Valinomycin & Calbiochem \\
\hline $\lg G$ (human) & Sigma Aldrich \\
\hline IgG (bovine) & Biorad \\
\hline Roti-Quant & Roth \\
\hline PEG4000 & Fluka \\
\hline Herring Sperm DNA & Promega \\
\hline Cytochrome $c$ (bovine) & Sigma Aldrich \\
\hline cis-Aconitic acid & Sigma Aldrich \\
\hline$n$-Dodecyl $\beta$-D-maltoside & Calbiochem \\
\hline Nonidet P40 (NP-40) & Fluka Biochemika \\
\hline X-Ray films & GE-Healthcare \\
\hline ECL Western blotting detection reagents & GE-Healthcare \\
\hline PVDF membrane & Milipore \\
\hline ADP & Sigma Aldrich \\
\hline Leupeptine & Sigma Aldrich \\
\hline Pefa bloc & Roche \\
\hline Oxaloacetic acid & Sigma Aldrich \\
\hline Malate & Sigma Aldrich \\
\hline Na-succinate & Sigma Aldrich \\
\hline Soybean Trypsin Inhibitor (SBTI) & Sigma Aldrich \\
\hline 2',7'-dichlorodihydrofluorescein diacetate $\left(\mathrm{H}_{2} \mathrm{DCFDA}\right)$ & Invitrogen \\
\hline Ergosterol & Sigma Aldrich \\
\hline
\end{tabular}




\begin{tabular}{lll}
\hline Table 2.2 Enzymes used in this study & & \\
\hline Product & Manufacturer & Application \\
\hline Zymolyase 20T & Seikagaku & Yeast cells' spheroplasting \\
Proetinase K & Roche & Protease protection assays \\
Creatin Kinase & Roche & In vitro import \\
Tryspin & Roth & In vitro import \\
TEV-Protease & Invitrogen & Protein purification \\
KOD Hot start DNA Polymerase & Novagen & PCR \\
Taq DNA Polymerase & USB & PCR \\
\hline
\end{tabular}

\section{Table 2.3 Plasmids used in this study}

\begin{tabular}{llll}
\hline Plasmid & Backbone & Insert & Reference \\
\hline p426TEF & - & - & Dualsystems Biotech \\
pMaD1 & pUG36 & - & This study \\
pMaD2 (pMaD1-RCF1) & pMaD1 & RCF1 & This study \\
pMaD10 (pMaD1-RCF1B) & pMaD1 & RCF1B & This study \\
pMaD11 (pMaD1-RCF1A) & pMaD1 & RCF1A & This study \\
pMaD12 (p426TEF-RCF1) & p426TEF & RCF1 & This study \\
pMaD13 (p426TEF-RCF1B) & p426TEF & RCF1B & This study \\
pMaD14 (p426TEF-RCF1A) & p426TEF & RCF1A & This study \\
\hline
\end{tabular}

\section{Table 2.4 Kit systems}

\begin{tabular}{ll}
\hline Product & Supplier \\
\hline Flexi Rabbit Reticulocyte Lysate System & Promega \\
mMESSAGE mMACHINE SP6 & Ambion \\
MEGAclear & Ambion \\
Wizard SV Gel and PCR Clean-Up System & Promega \\
Wizard Plus SV Minipreps DNA Purification System & Promega \\
Rapid DNA Ligation Kit & Fermentas \\
SYBR Green qPCR Reaction Kit & Clontech \\
\hline
\end{tabular}




\begin{tabular}{|c|c|c|}
\hline Strain & Genotype & Reference \\
\hline YPH499 & MATa ade2-101 his3-4200 leu2-41 ura3-52 trp1- 463 lys2-801 & $\begin{array}{l}\text { Sikorski and Hieter, } \\
1989\end{array}$ \\
\hline BY4741 (WT) & MATa his3 $\Delta 1$ leu2 $\Delta 0$ met15 $\Delta 0$ ura3 $\Delta 0$ & Eurocsarf \\
\hline 777-A3 & $M A T \alpha$, ade 1 op 1 & Netter et al., 1982 \\
\hline $\operatorname{cor1}^{\text {TAP }}$ & 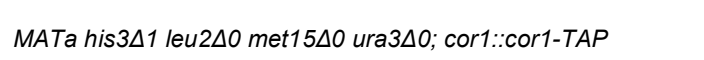 & Euroscarf \\
\hline MVY2 (rcf2 $\Delta)$ & $\begin{array}{l}\text { MATa ade2-101 his3- } \Delta 200 \text { leu2- } \Delta 1 \text { ura3-52 trp1- } \triangle 63 \text { lys2-801; } \\
\text { YNR018W::HIS3MX6 }\end{array}$ & This study \\
\hline yMaD2 $(r c f 1 \Delta)$ & $\begin{array}{l}\text { MATa, ade2-101 his3- } \triangle 200 \text { leu2- } \Delta 1 \text { ura3-52 trp1- } \Delta 63 \text { lys2-801; } \\
\text { YML030w::IoxP }\end{array}$ & This study \\
\hline AFY10 (cyt1 $\Delta)$ & $\begin{array}{l}\text { MATa, ade2-101 his3- } \triangle 200 \text { leu2- } \Delta 1 \text { ura3-52 trp1- } \triangle 63 \text { lys2-801; } \\
\text { YOR065w::HISMX6 }\end{array}$ & Frazier thesis \\
\hline AFY11 $(\operatorname{cox} 4 \Delta)$ & $\begin{array}{l}\text { MATa, ade2-101 his3- } \triangle 200 \text { leu2- } \Delta 1 \text { ura3-52 trp1- } \Delta 63 \text { lys2-801; } \\
\text { YGL187c::HISMX6 }\end{array}$ & Frazier et al., 2006 \\
\hline MVY5 $(\cos 13 \Delta)$ & 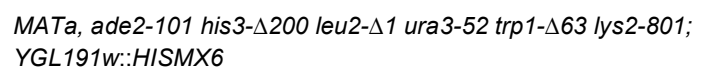 & This study \\
\hline MVY4 (rcf2 $\left.{ }^{Z Z}\right)$ & $\begin{array}{l}\text { MATa ade2-101 his3- } \Delta 200 \text { leu2- } \Delta 1 \text { ura3-52 trp1- } \Delta 63 \text { lys2-801; } \\
\text { rcf2::rcf2-ZZ }\end{array}$ & This study \\
\hline HCY01 $\left(\operatorname{cox} 13^{Z Z}\right)$ & $\begin{array}{l}\text { MATa, ade2-101 his3- } \triangle 200 \text { leu2- } \Delta 1 \text { ura3-52 trp1- } \Delta 63 \text { lys2-801; } \\
\text { cox13::cox13-ZZ }\end{array}$ & This study \\
\hline $\mathrm{HCYO4}\left(\operatorname{cox} 4^{\mathrm{ZZ}}\right)$ & $\begin{array}{l}\text { MATa, ade2-101 his3- }-200 \text { leu2- } \Delta 1 \text { ura3-52 trp1- } 663 \text { lys2-801; } \\
\text { cox4::cox4-ZZ }\end{array}$ & This study \\
\hline $\operatorname{MVY9}\left(\operatorname{cor} 1^{\mathrm{ZZ}}\right)$ & $\begin{array}{l}\text { MATa, ade2-101 his3- } \Delta 200 \text { leu2- } \Delta 1 \text { ura3-52 trp1- } \Delta 63 \text { lys2-801; } \\
\text { cor1::cor1-ZZ }\end{array}$ & This study \\
\hline MVY6 $(r c f 2 \uparrow)$ & $\begin{array}{l}\text { MATa, ade2-101 his3- } \triangle 200 \text { leu2- } \Delta 1 \text { ura3-52 trp1- } \Delta 63 \text { lys2-801; } \\
\text { PGPD-YNR018W }\end{array}$ & This study \\
\hline $\mathrm{G} 421\left(\operatorname{cox} 1^{-}\right)$ & MAT $\alpha$, ade 1 op $1 ; \operatorname{cox} 1-G 421$ & Netter et al., 1982 \\
\hline DaMY02 (taz1 1$)$ & $\begin{array}{l}\text { MATa, ade2-101 his3- } \Delta 200 \text { leu2- } \Delta 1 \text { ura3-52 trp1- } \Delta 63 \text { lys2-801; } \\
\text { YPR140w::kanMX4 }\end{array}$ & Brandner et al., 2005 \\
\hline$r c f 1 \Delta+p R C F 1$ & $\begin{array}{l}\text { MATa, ade2-101 his3- } \triangle 200 \text { leu2- } \Delta 1 \text { ura3-52 trp1- } 663 \text { lys2-801; } \\
\text { YML030w::IoxP + P426-TEF-RCF1 }\end{array}$ & This study \\
\hline $\operatorname{rcf1} 1 \Delta+\mathrm{phRCF} 1 \mathrm{~A}$ & $\begin{array}{l}\text { MATa, ade2-101 his3- } \triangle 200 \text { leu2- } \triangle 1 \text { ura3-52 trp1- } \Delta 63 \text { lys2-801; } \\
\text { YML030w::loxP + P426-TEF-hRCF1A }\end{array}$ & This study \\
\hline$r c f 1 \Delta+p h R C F 1 B$ & $\begin{array}{l}\text { MATa, ade2-101 his3- } \triangle 200 \text { leu2- } \triangle 1 \text { ura3-52 trp1- } 663 \text { lys2-801; } \\
\text { YML030w::loxP + P426-TEF-hRCF1B }\end{array}$ & This study \\
\hline
\end{tabular}




\begin{tabular}{|c|c|}
\hline Primary antibodies (yeast) & Reference \\
\hline$\alpha-\operatorname{Rcf1}$ & AG Rehling (2321) \\
\hline$\alpha-\operatorname{Rcf2}$ & AG Rehling (2319) \\
\hline$\alpha-\operatorname{Cox} 1$ & AG Rehling (1539) \\
\hline$\alpha-\operatorname{Cox} 2$ & AG Rehling (1948) \\
\hline$\alpha-\operatorname{Cox} 3$ & Abcam \\
\hline$\alpha-\operatorname{Cox} 4$ & AG Rehling (578) \\
\hline$\alpha-\operatorname{Cox} 5 a$ & AG Rehling (1540) \\
\hline$\alpha-\operatorname{Cox} 6$ & AG Rehling (2016) \\
\hline$\alpha-\operatorname{Cox} 13$ & AG Rehling (1543) \\
\hline$\alpha-\operatorname{Cox} 14$ & AG Rehling (1544) \\
\hline$\alpha-C y t 1$ & AG Rehling (540) \\
\hline$\alpha-\operatorname{Rip} 1$ & AG Rehling (543) \\
\hline$\alpha-Q c r 8$ & AG Rehling (1037) \\
\hline$\alpha-A t p 5$ & AG Rehling (1546) \\
\hline$\alpha-F_{1} \beta$ & AG Rehling (861) \\
\hline$\alpha-\operatorname{Tim} 10$ & AG Rehling (217) \\
\hline$\alpha-\operatorname{Tim} 21$ & AG Rehling (258) \\
\hline$\alpha-\operatorname{Tim} 22$ & AG Rehling (164) \\
\hline$\alpha-\operatorname{Tim} 44$ & AG Rehling (127) \\
\hline$\alpha$-Tom40 & AG Rehling (168) \\
\hline$\alpha$-Tom70 & AG Rehling (657) \\
\hline$\alpha-H s p 70$ & AG Rehling (119) \\
\hline$\alpha-A \operatorname{co} 1$ & AG Rehling (945) \\
\hline$\alpha-$ Mio10 & AG Rehling (3459) \\
\hline$\alpha$-ProteinA & Sigma Aldrich \\
\hline Primary antibodies (human) & Reference \\
\hline$\alpha-R C F 1 a$ & Santa Cruz Biotech \\
\hline$\alpha-R C F 1 b$ & AG Rehling (3161) \\
\hline$\alpha-\operatorname{CoX} 1$ & AG Rehling (2034) \\
\hline$\alpha-\operatorname{Cox} 6 \mathrm{~A} 1$ & AG Rehling (3283) \\
\hline$\alpha$-Rieske & AG Rehling (1512) \\
\hline$\alpha$-NDUFA9 & AG Rehling (1524) \\
\hline$\alpha-F_{1} \beta$ & Invitrogen \\
\hline$\alpha$-TIM23 & AG Rehling (1526) \\
\hline$\alpha$-TOM70 & AG Rehling (3280) \\
\hline$\alpha$-TACO1 & AG Rehling (3627) \\
\hline$\alpha$-Cyclophilin D & Abcam \\
\hline$\alpha-\operatorname{COX}$ & Invitrogen \\
\hline$\alpha-F_{1} F_{0}-$ ATPase & Invitrogen \\
\hline Secondary antibodies & Reference \\
\hline GaR-HRP & Dianova \\
\hline Alexa Fluor 488 & Invitrogen \\
\hline Alexa Fluor 594 & Invitrogen \\
\hline
\end{tabular}




\subsection{E. coli- media and growth}

\subsubsection{Media for $E$. coli}

LB medium: $1 \%(\mathrm{w} / \mathrm{v})$ bacto-tryptone, $0.5 \%(\mathrm{w} / \mathrm{v})$ yeast extract, $1 \%(\mathrm{w} / \mathrm{v}) \mathrm{NaCl}$ LB-Amp medium: LB medium supplemented with $100 \mu \mathrm{g} / \mathrm{ml}$ of ampicillin

Described media solutions were used to prepare liquid cultures. To prepare LB or LB-Amp solid media, $1.5 \%(\mathrm{w} / \mathrm{v})$ bacto-agar was added to the liquid media solutions and autoclaved for $20 \mathrm{~min}$ at $120^{\circ} \mathrm{C}$. Ampicillin was added after media had cooled to $50^{\circ} \mathrm{C}$.

\subsubsection{Cultivation of $E$. coli}

E.coli cells were grown in LB medium. For selection of plasmids, carrying an $A m p^{R}$ marker, LB-Amp medium was used. Liquid cultures were inoculated from a plate and incubated at $37^{\circ} \mathrm{C}$ while shaking. The growth of E.coli cultures was monitored by $\mathrm{OD}_{600}$ measurements.

For E.coli strain storage, cryo-stocks were prepared: $1 \mathrm{ml}$ of bacterial culture was supplemented with $200 \mu \mathrm{l}$ of sterile $80 \%$ glycerol and stored at $-80^{\circ} \mathrm{C}$.

\subsection{S. cerevisiae - media and growth}

\subsubsection{Media for S. cerevisiae}

YP medium: $1 \%(\mathrm{w} / \mathrm{v})$ yeast extract, $2 \%(\mathrm{w} / \mathrm{v})$ bacto-peptone.

YPD medium: YP medium supplemented with $2 \%$ glucose.

YPGal medium: YP medium supplemented with $2 \%$ galactose.

YPG medium: YP medium supplemented with $3 \%$ glycerol.

Selective medium: $0.67 \%(\mathrm{w} / \mathrm{v})$ yeast nitrogen base w/o amino acids, $0.07 \%(\mathrm{w} / \mathrm{v})$ drop-out mix (a mixture containing an equal amount of all amino acids; for selection one auxotrophic marker, the corresponding amino acid was left out), supplemented with $2 \%(\mathrm{w} / \mathrm{v})$ glucose (SD) or $3 \%$ glycerol (SG).

Described media solutions were used to prepare liquid cultures. To prepare solid media, $2 \%(\mathrm{w} / \mathrm{v})$ bacto-agar was added to liquid media before autoclaving at $120^{\circ} \mathrm{C}$ for $20 \mathrm{~min}$.

Semisynthetic galactose medium, SSG-TAE (Dagsgaard et al., 2001), contained per liter: galactose 
$10 \mathrm{~g}$, yeast nitrogen base $3 \mathrm{~g}, \mathrm{KH}_{2} \mathrm{PO}_{4} 1 \mathrm{~g},\left(\mathrm{NH}_{4}\right)_{2} \mathrm{SO}_{4} 0.8 \mathrm{~g}, \mathrm{MgSO}_{4} \cdot 7 \mathrm{H}_{2} \mathrm{O} 0.7 \mathrm{~g}, \mathrm{NaCl} 0.5 \mathrm{~g}, \mathrm{CaCl}_{2}$ $0.4 \mathrm{~g}, \mathrm{FeCl}_{3} 5 \mu \mathrm{g}, 0.1 \%(\mathrm{v} / \mathrm{v})$ Tween-80, ergosterol $20 \mu \mathrm{g}, 40 \mu \mathrm{g}$ amino acids and nucleotides.

\subsubsection{S. cerevisiae growth}

Liquid cultures were inoculated from plates and were grown in the appropriate liquid medium at $30^{\circ} \mathrm{C}$ while shaking at $140 \mathrm{rpm}$. Cell density was monitored by $\mathrm{OD}_{600}$ measurements. For the mitochondrial isolation, cells were propagated for 3 days, and harvested at an $\mathrm{OD}_{600}$ of 2-2.5.

\subsubsection{Fermentor cultivation of yeast cells}

Cells were grown in a BIOSTAT Aplus (Sartorius) fermentor system. The temperature $\left(30^{\circ} \mathrm{C}\right), \mathrm{pH}(7.0)$ and stiring speed $(300 \mathrm{rpm})$ were controlled automatically by the fermentor system software. The dissolved oxygen concentration in the fermentor was monitored with a Clark type oxygen electrode (Hamilton). Midexponential precultures were used to inoculate the fermentor to a low cell density. Cells were grown either aerobically ( $20 \%$ oxygen) or hypoxic ( $0 \%$ oxygen), while and the growth was monitored every four hours via measuring $\mathrm{OD}_{600}$.

\subsubsection{Yeast cryo-stocks}

For storage of yeast strains, $0.75 \mathrm{ml}$ of yeast culture was supplemented with $0.75 \mathrm{ml}$ of sterile YPAD media (1\% (w/v) yeast extract, $2 \%(\mathrm{w} / \mathrm{v})$ bacto-peptone, $2 \%(\mathrm{w} / \mathrm{v})$ glucose, $30 \mu \mathrm{g} / \mathrm{ml}$ adenine sulfate, $15 \%$ ( $\mathrm{v} / \mathrm{v}$ ) glycerol).

\subsubsection{Dilution assay}

To determine the growth characteristics of different yeast strains, a dilution assay was performed. Cells were grown in YPD/SD medium to exponential phase, washed two times with sterile water and diluted in sterile water to an $\mathrm{OD}_{600}$ of 0.5 . Cells were than diluted in 10-fold increments. From each dilution $5 \mu \mathrm{l}$ was spotted onto the indicated plate. Plates were incubated at $30^{\circ} \mathrm{C}$ for $2-5$ days. To analyse the growth characteristics under low oxygen conditions, plates were incubated in an INVIVO 400 Hypoxic Workstation (Ruskinn Life Science), at $30^{\circ} \mathrm{C}, 1 \%$ oxygen, for 48 hours, 
followed by an incubation at $30^{\circ} \mathrm{C}, 20 \%$ oxygen, for 48 hours. Growth was documented using a CCD camera (LAS 3000, Fujifilm).

\subsection{Culture of human cell lines}

HEK-293T and U2-OS cells were grown in Dulbecco's Modified Eagle Medium supplemented with $10 \%(\mathrm{w} / \mathrm{v})$ fetal bovine serum (Gibco, Invitrogen). Growth was performed at $37^{\circ} \mathrm{C}$ under $5 \% \mathrm{CO}_{2}$ atmosphere in $75 \mathrm{~cm}^{2}$ bottles or $175 \mathrm{~cm}^{2}$ dishes (Nunc). After reaching $80-85 \%$ confluency, cells were split by detaching from the surface using PBS (137 mM NaCl, $2.7 \mathrm{mM} \mathrm{KCl}, 12 \mathrm{mM}$ potassium phosphate, $\mathrm{pH}$ 7.4), supplemented with $1 \mathrm{mM}$ EDTA. Cells were washed with PBS, sedimented at $200 \mathrm{~g}$ for $5 \mathrm{~min}$ and seeded on a new plate or flask with fresh culture medium.

\subsection{Molecular biology methods}

\subsubsection{Determination of nucleic acid concentrations}

To determine DNA and RNA concentrations, NanoVue spectrophotometer (GEHealthcare) was used. One optical unit $\left(\mathrm{OD}_{260}=1\right)$ corresponds to $50 \mu \mathrm{g} / \mathrm{ml}$ DNA or $40 \mu \mathrm{g} / \mathrm{ml}$ RNA.

\subsubsection{DNA amplification by Polymerase Chain Reaction (PCR)}

DNA sequences were amplified by a polymerase chain reaction (PCR). Two thermostable DNA polymerases were used: Taq (isolated from Thermus aquaticus) (Affymetrix) and KOD Hot Start DNA Polymerase (recombinant form of Thermococcus kodakaraensis KOD1 DNA polymerase) (Novagen). PCR reactions were performed in $50 \mu \mathrm{l}$ scale, using $25 \mathrm{ng}$ plasmid DNA or $200 \mu \mathrm{g}$ of yeast genomic DNA as a template.

The following program was used:

1) $95^{\circ} \mathrm{C}, 5 \mathrm{~min} \quad$ Polymerase activation and complete denaturation of DNA

2) 30-35 cycles: $\quad 30$ seconds, $95^{\circ} \mathrm{C}$; denaturation of DNA

30 seconds, $45-65^{\circ} \mathrm{C}$; annealing of primers

30-180 seconds, $72^{\circ} \mathrm{C}$; extension of DNA (duration of this 
step is determined by the length of the DNA fragment to be amplified and the DNA polymerase used

- Taq polymerase: 60 seconds $/ 1 \mathrm{kbp}$

- KOD Hot Start DNA Polymerase: 30 seconds/1kbp

3) $72^{\circ} \mathrm{C}, 10 \mathrm{~min} \quad$ Completion of the last reaction

\subsubsection{RT-PCR analysis}

RNA for RT-PCR analysis was extracted from different organs of FVB mice using TRIzol Reagent (Invitrogen). Reverse transcription was performed with first strand cDNA synthesis kit (Fermentas, St. Leon-Rot). $2 \mu \mathrm{g}$ of RNA was used for the reaction. mRNA levels were quantified using MX3000P light cycler (Stratagene) and SYBR Green qPCR reaction kit (Clontech). For each reaction $1 \mu \mathrm{l}$ of cDNA was used. The relative quantification was performed by normalizing the target gene to the houskeeper gene, S12. All analyses were done in five replicates.

Primers used for quantification:

mS12 for 5'-GAAGCTGCCAAGGCCTTAGA-3'

mS12 rev 5'-AACTGCAACCAACCACCTTC-3'

mRCF1a for 5'-GCCCAGGCCTCACGTCTGACA-3'

mRCF1a rev 5'-GAGTCTTCCGAGACGCGGGGT-3'

mRCF1b for 5'-CCTATAGGCTGTCTGGGGACGG-3'

mRCF1b rev 5'-CATGGCGCTTTAAGACCCGGC-3'.

\subsubsection{Agarose gel DNA electrophoresis}

To separate DNA fragments according to their size, electrophoresis in a horizontal agarose gel was used. Agarose 1\% (w/v) was dissolved in TAE buffer (40 mM TrisBase, $0.14 \%(\mathrm{v} / \mathrm{v})$ acetic acid, $1 \mathrm{mM}$ EDTA, $\mathrm{pH}$ 8.0). To allow visualization of DNA in the gel under UV light, the agarose solution was supplemented with $0.1 \mu \mathrm{g} / \mathrm{ml}$ ethidium bromide. Samples were mixed with a loading buffer $(4 \mathrm{x}$ stock: $40 \%$ saccharose, $1 \%$ OrangeG). Electrophoresis was performed in Mini-Sub Cell GT chambers (Biorad) in TAE buffer for $30 \mathrm{~min}$ at $120 \mathrm{~V}$. In each run a molecular weight markers (Gene Ruler-Fermentas) was used. DNA bands were visualized by an UVtransilluminator. 


\subsubsection{Isolation of DNA from agarose gels}

DNA fragments to be further processed were excised from the gel under UV light. DNA was extracted from the gel using Wizard SV Gel and PCR Clean-Up System (Promega). After extraction of the DNA from the gel 1-2 $\mu$ of the purified DNA was loaded on the agarose gel, to verify the efficiency of purification. DNA purified in this way was stored at $-20^{\circ} \mathrm{C}$.

\subsubsection{Enzymatic manipulation of DNA}

PCR products and plasmid DNA for preparative purposes was digested using Fast Digest restriction enzymes (Fermentas). Obtained DNA fragments were analyzed by agarose gel electrophoresis, extracted from the gel and used in subsequent ligation reactions.

Ligation reactions were performed using a Rapid DNA Ligation Kit (Fermentas) following which reactions were directly transformed into chemically competent E.coli XL1 Blue cells. Verification of the clones was performed by restriction analysis and sequencing.

\subsubsection{Transformation of $E$. coli cells}

\subsubsection{Preparation of transformation competent E.coli cells with $\mathrm{CaCl}_{2}$}

A small culture $(5 \mathrm{ml})$ of LB medium was inoculated with a single colony of the corresponding strain and incubated over night at $37^{\circ} \mathrm{C}$ while shaking at $140 \mathrm{rpm}$. The following day, $500 \mathrm{ml}$ of LB medium was inoculated with $5 \mathrm{ml}$ of an overnight culture and were susequently grown $\left(37^{\circ} \mathrm{C} / 140 \mathrm{rpm}\right)$ until they had reached mid-log phase $\left(\mathrm{OD}_{600}=0.5\right)$. Cultures were chilled on ice for $10 \mathrm{~min}$ and then centrifuged $(5000 \mathrm{~g}$, $5-10 \mathrm{~min}, 4^{\circ} \mathrm{C}$ ). After centrifugation, the cell pellet was resuspended in $250 \mathrm{ml}$ of cold, sterile $50 \mathrm{mM} \mathrm{CaCl}_{2}$ and incubated on ice for $15 \mathrm{~min}$, followed by an additional centrifugation step (5000 g, 5-10 min, $4^{\circ} \mathrm{C}$ ). E. coli cells were resuspended in $25 \mathrm{ml}$ of $50 \mathrm{mM} \mathrm{CaCl} 2$, aliquoted and stored at $-80^{\circ} \mathrm{C}$. 


\subsubsection{Transformation of $\mathrm{CaCl}_{2}$-competent $E$. coli cells by heat shock}

$100 \mu$ aliquots of $\mathrm{CaCl}_{2}$ competent $E$. coli cells were thawed on ice. The DNA (10$100 \mathrm{ng}$ ) was added to the $E$. coli cells and incubated on ice for $15 \mathrm{~min}$. The samples were heat-shocked at $42^{\circ} \mathrm{C}$ for $2 \mathrm{~min}$, and then placed on ice for $2 \mathrm{~min}$. Cells were diluted with $1 \mathrm{ml} \mathrm{LB}$ medium and incubated at $37^{\circ} \mathrm{C}$ for $30-60 \mathrm{~min}$, while shaking. After a brief centrifugation and the removal of most of the medium, cells were plated on LB plates containing the corresponding antibiotic.

\subsubsection{Sequencing of DNA}

Sequencing of DNA was performed via the Sanger method using the BigDye Terminator v1.1 Cycle Sequencing Kit. The sequencing reaction was performed in a $10 \mu \mathrm{l}$ scale, containing $300 \mathrm{ng}$ plasmid, $8 \mathrm{pmol}$ primer, $1.5 \mu \mathrm{l}$ sequencing mix and 1.5 $\mu \mathrm{l}$ sequencing buffer. Following the sequencing reaction $\left(25\right.$ cycles: $96^{\circ} \mathrm{C}, 10 \mathrm{sec}$; $\left.55^{\circ} \mathrm{C} 15 \mathrm{sec} ; 60^{\circ} \mathrm{C}, 4 \mathrm{~min}\right), 1 \mu \mathrm{l}$ of $125 \mathrm{mM}$ EDTA and $1 \mu \mathrm{l} 3 \mathrm{M}$ sodium acetate was added and the DNA was precipitated through the addition of $50 \mu 100 \%$ ethanol. Subsequently the DNA was pelleted (20000 g, $15 \mathrm{~min}$ ), washed with $70 \%$ ethanol and dried in a vacuum concentrator. For sequencing the DNA was resuspended in $15 \mu \mathrm{Hi}$-Di-formamide and analyzed using a Genetic Analyzer 3100 (Applied Biosystems) in the Göttingen Center for Molecular Biosciences.

\subsubsection{Transformation of $S$. cerevisiae cells by lithium acetate method}

Transformation of yeast cells, following treatment with lithium acetate was first reported by lto et al. (1983). Yeast transformation with plasmids and integration constructs in this study was performed according to Gietz and Schiestl (2007). Integration cassettes amplified by PCR were chromosomally integrated in yeast cells. Yeast strains designated for transformation were grown over night in $10 \mathrm{ml}$ of YPD medium. On following day the over night culture was diluted to $\mathrm{OD}_{600}$ of 0.1 and cells were grown at $30^{\circ} \mathrm{C} / 120 \mathrm{rpm}$, until they reached an $\mathrm{OD}_{600}$ of 0.6 . Cells were harvested by centrifugation (7000 g, $5 \mathrm{~min}$, RT), washed once with sterile water and once with $100 \mathrm{mM}$ lithium acetate. After the washing steps, cells were resuspended 
in $1 \mathrm{ml}$ of $100 \mathrm{mM}$ lithium acetate. For each transformation $100 \mu \mathrm{l}$ of the cell suspension was mixed with $10 \mu \mathrm{l}$ of single stranded salmon sperm DNA, 0.1-10 $\mu \mathrm{g}$ of DNA to be transformed and $600 \mu \mathrm{l}$ of LiAc-PEG mixture (10 mM Tris-HCl $(\mathrm{pH} 7.5)$, $1 \mathrm{mM}$ EDTA, $100 \mathrm{mM}$ lithium acetate, $40 \%$ (w/v) PEG 4000). The mixture was vortexed and then incubated for $20-30 \mathrm{~min}$ at $30^{\circ} \mathrm{C}$. After incubation, $75 \mu \mathrm{l}$ of DMSO was added and the mixture was incubated for a further $15 \mathrm{~min}$ at $42^{\circ} \mathrm{C}$. The cells were harvested by centrifugation (7000 g, $5 \mathrm{~min}, \mathrm{RT}$ ), washed with sterile water and resuspended in $5 \mathrm{ml}$ of YPD and incubated for 2-4 hours in an end-over-end shaker at $30^{\circ} \mathrm{C}$. The cells were then centrifuged $(7000 \mathrm{~g}, 5 \mathrm{~min}, \mathrm{RT})$, and washed twice with sterile water. After washing, cells were resuspended in $100 \mu \mathrm{l}$ of sterile water and spread on the appropriate selective plates. The plates were incubated at $30^{\circ} \mathrm{C}$.

\subsubsection{Preparation of yeast genomic DNA}

\subsubsection{Small scale preparation of yeast genomic DNA}

YPD medium $(5 \mathrm{ml})$ was inoculated with $S$. cerevisiae cells and incubated until the $\mathrm{OD}_{600}$ reached $0.5-0.7$. Cells were harvested by centrifugation $\left(7000 \mathrm{~g}, 5 \mathrm{~min}, 4^{\circ} \mathrm{C}\right)$, resuspended in $150 \mu$ solution, containing $50 \mathrm{mM}$ Tris-HCl (pH 7.5), $10 \mathrm{mM}$ EDTA, $0.3 \%(\mathrm{v} / \mathrm{v}) \beta$-mercaptoethanol and $0.5 \mathrm{mg} / \mathrm{ml}$ zymolyase, and incubated at $37^{\circ} \mathrm{C}$ for 1 hour. Then, $1.1 \%(\mathrm{w} / \mathrm{v}) \mathrm{SDS}$ and $2.2 \mathrm{M} \mathrm{NH}_{4}$-acetate was added and the samples were vortexed and incubated at $-20^{\circ} \mathrm{C}$ for $15 \mathrm{~min}$. After incubation, samples were centrifuged $\left(20000 \mathrm{~g}, 15 \mathrm{~min}, 4^{\circ} \mathrm{C}\right)$ and $180 \mu \mathrm{l}$ of the supernatant were transferred to a new tube, mixed with $120 \mu \mathrm{l}$ of isopropanol and centrifuged $\left(20000 \mathrm{~g}, 15 \mathrm{~min}, 4^{\circ} \mathrm{C}\right.$ ). The pellet was washed with 70\% ice-cold ethanol, dried and resuspended in 20-30 $\mu \mathrm{l}$ of TE buffer (10 mM Tris-HCl ( $\mathrm{pH} \mathrm{8.0)}$ and 1 mM EDTA).

\subsubsection{Large scale preparation of yeast genomic DNA}

A yeast culture $(50 \mathrm{ml})$ was grown over night at $30^{\circ} \mathrm{C}$, while shaking. On the following day, the culture was diluted with $100-150 \mathrm{ml}$ of fresh YPD media and grown to an $\mathrm{OD}_{600}$ of $6-8$. Cells were harvested by centrifugation $\left(7000 \mathrm{~g}, 5 \mathrm{~min}, 4^{\circ} \mathrm{C}\right)$ and resuspended in $2 \mathrm{ml}$ of ice-cold $0.9 \mathrm{M}$ sorbitol, and then centrifuged again $(7000 \mathrm{~g}, 5$ 
$\min , 4^{\circ} \mathrm{C}$ ). The pellet was resuspended in $1 \mathrm{ml}$ of buffer, containing $1 \mathrm{M}$ sorbitol, 50 $\mathrm{mM}$ potassium phosphate $(\mathrm{pH} 7.5), 14 \mathrm{mM} \beta$-mercaptoethanol and $500 \mu \mathrm{g} / \mathrm{ml}$ zymolyase and incubated at $30^{\circ} \mathrm{C}$ with moderate shaking, until the culture became to transparent. Following cell lysis, the sample was centrifuged $\left(5000 \mathrm{~g}, 2 \mathrm{~min}, 4^{\circ} \mathrm{C}\right.$ ). The pellet was resuspended in $1 \mathrm{ml}$ solution, containing $0.2 \%(\mathrm{w} / \mathrm{v}) \mathrm{SDS}, 50 \mathrm{mM}$ EDTA, $\mathrm{pH} 7.9$, and then $0.5 \mathrm{M}$ potassium acetate was added, mixed and incubated on ice for $30 \mathrm{~min}$, followed by centrifugation. After centrifugation, the supernatant was transferred to a new tube, mixed with $1 \mathrm{ml}$ of $100 \%$ ice-cold ethanol and centrifuged ( $20000 \mathrm{~g}, 12 \mathrm{~min}, 4^{\circ} \mathrm{C}$ ). DNA was resuspended in $1 \mathrm{ml}$ of TE buffer, and then $30 \mu \mathrm{g} / \mathrm{ml}$ RNase was added, followed by incubation at $37^{\circ} \mathrm{C}$ for $1-2$ hours. After extraction of DNA by phenol/chloroform, $1 \mathrm{ml}$ of $100 \%$ ice-cold ethanol was added and centrifuged $\left(20000 \mathrm{~g}, 12 \mathrm{~min}, 4^{\circ} \mathrm{C}\right)$. The pellet was washed with $1-2 \mathrm{ml}$ of $70 \%$ ice-cold ethanol, dried and resuspended in $100 \mu$ l of TE buffer.

\subsection{Biochemical Methods}

\subsubsection{Determination of protein concentrations}

Protein concentration determination was performed according to the Bradford assay. Protein solutions $(5,10$ and $15 \mu \mathrm{l})$ were diluted with $1 \mathrm{ml}$ of 1:10 diluted "Roti-Quant" reagent (Roth) and incubated for $15 \mathrm{~min}$ at room temperature. The absorbance was measured at $600 \mathrm{~nm}$ (BioPhotometer (Eppendorf)) using a $1 \mathrm{~cm}$-path length cuvette. Protein concentrations were determined according to a standard curve obtained using known amounts of the bovine $\operatorname{lgG}$ (BioRad) as standards.

\subsubsection{Whole cell yeast extraction}

Extraction of total proteins from yeast cells was performed as described (Yaffe and Schatz, 1984) with minor modifications. Yeast strains were inoculated in $5 \mathrm{ml}$ YPD or selective medium and incubated over night at $30^{\circ} \mathrm{C}$ in an end-over-end shaker. On the next day yeast cells were harvested by centrifugation at $7000 \mathrm{~g}$ for $2 \mathrm{~min}$ and resuspended in $1 \mathrm{ml}$ of water. Cells were lysed by addition of $250 \mathrm{mM}$ sodium hydroxide and $15 \mathrm{mM}$ of $\beta$-mercaptoethanol. This mixture was incubated on ice for 
$10 \mathrm{~min}$ and the proteins were precipitated through the addition of $6 \%(\mathrm{w} / \mathrm{v})$ trichloroacetic acid and incubation for $10 \mathrm{~min}$ on ice, and then centrifuged at (20000 $\mathrm{g}, 2 \mathrm{~min}, 4^{\circ} \mathrm{C}$ ). Pellets were resuspended in $50 \mu \mathrm{l}$ of $1 \times$ SDS sample buffer, incubated for $5 \mathrm{~min}$ at $95^{\circ} \mathrm{C}$ and analyzed by SDS-PAGE and immunodecoration.

\subsubsection{SDS-PAGE}

Proteins were separated according to their molecular weights under denaturing conditions by one-dimensional vertical SDS-Polyacrylamide gel electrophoresis (SDS-PAGE) (Laemmli, 1970). The concentrations of acrylamide and bis-acrylamide in the separating gel were selected according to the molecular sizes of the proteins of interests. The amount of protein loaded was between 10 and $100 \mu \mathrm{g}$ per lane. Prior to loading, protein samples were resuspended in $1 \times$ SDS-sample buffer with or without $0.05 \%(\mathrm{v} / \mathrm{v}) \beta$-mercaptoethanol and incubated at $95^{\circ} \mathrm{C}$ for $5 \mathrm{~min}$. For highly hydrophobic proteins, the incubation step was omitted. Electrophoresis was performed at 25-35 mA, $230 \mathrm{~V}$, for gels with dimensions of $14 \times 9 \times 0,1 \mathrm{~cm}$ and 25 mA, $200 \mathrm{~V}$ for mini gels (Mini-PROTEAN II, BioRad) at 25 mA, $200 \mathrm{~V}$. Broad Range (BioRad) protein molecular weight markers were used.

Separating gel: 12-15\% (w/v) acrylamide, 0.32-0.4\% (w/v) bis-acrylamide, $375 \mathrm{mM}$ Tris- $\mathrm{HCl}(\mathrm{pH} 8.8)$, $0.1 \%(w / v)$ SDS, $0.05 \%(w / v)$ APS, $0.05 \%$ (v/v) TEMED

Stacking gel: 4\% (w/v) acrylamide, $0.1 \%(\mathrm{w} / \mathrm{v})$ bis-acrylamide, $80 \mathrm{mM}$ Tris- $\mathrm{HCl}(\mathrm{pH} 6.8), 0.1 \%(\mathrm{w} / \mathrm{v})$ SDS, $0.05 \%(\mathrm{w} / \mathrm{v})$ APS, $0.2 \%(\mathrm{v} / \mathrm{v})$ TEMED

SDS-electrophoresis buffer: $25 \mathrm{mM}$ Tris, $191 \mathrm{mM}$ glycine, 0.1\% (w/v) SDS

1 x SDS-sample-buffer: $60 \mathrm{mM}$ Tris-HCI (pH 6.8), 2\% (w/v) SDS, 10\% (v/v) glycerol, 0.05\% (v/v) $\beta$ mercaptoethanol, $0.01 \%(\mathrm{w} / \mathrm{v})$ Bromophenol blue.

\subsubsection{Urea-gel electrophoresis}

To obtain higher resolution of low molecular weight proteins, urea-gel electrophoresis was employed. The same system as for SDS-PAGE was used, just that gel mixtures and electrophoresis buffer were different. Electrophoresis was performed at $30 \mathrm{~mA}(4-5 \mathrm{~h})$.

Separating gel: $17.5 \%$ acrylamide, $0.2 \%$ bis-acrylamide, $33 \%(\mathrm{w} / \mathrm{v})$ urea, $700 \mathrm{mM}$ Tris- $\mathrm{HCl},(\mathrm{pH} 8.8)$, $0.1 \%(w / v)$ SDS, $8 \mathrm{mM} \mathrm{NaCl}, 0.1 \%(\mathrm{w} / \mathrm{v})$ APS, $0.05 \%$ TEMED 
Stacking gel: $5.5 \%$ acrylamide, $0.07 \%$ bis-acrylamide, $22 \%(\mathrm{w} / \mathrm{v})$ urea, $0.1 \mathrm{M}$ Tris- $\mathrm{HCl}(\mathrm{pH} 6.8), 0.1 \%$ SDS, $0,1 \%$ APS, $0.05 \%$ TEMED

Urea-electrophoresis buffer: $50 \mathrm{mM}$ Tris, $191 \mathrm{mM}$ glycine, 0.1\% (w/v) SDS

\subsubsection{BN-PAGE}

To separate proteins under non-denaturing (native) conditions, BN-PAGE (Schägger and von Jagow, 1991) was employed. For this purpose, mitochondria were pelleted $\left(20000 \mathrm{~g}, 10 \mathrm{~min}, 4^{\circ} \mathrm{C}\right)$ and resuspended in solubilization buffer $(1 \mu \mathrm{l} / \mu \mathrm{g}$ of protein). Solubilization was performed at $4^{\circ} \mathrm{C}$ for $15 \mathrm{~min}$. After a clarifying spin $(20000 \mathrm{~g}, 10$ min, $4^{\circ} \mathrm{C}$ ), 10 fold sample buffer was added to the soluble fraction, mixed and loaded onto a $4-10 \%, 4-13 \%$ or $6-10 \%$ gradient gel with a $4 \%$ stacking gel. Electrophoresis was performed using the SE600 Ruby System (GE-Healthcare), at $4^{\circ} \mathrm{C}$, starting with the voltage of $200 \mathrm{~V}, 15 \mathrm{~mA}$ for $1 \mathrm{~h}$ and then shifted to $600 \mathrm{~V}$. During the electrophoresis, cathode buffer with Coomassie Brilliant Blue G-250 and anode buffer were used. If the gels were designated for Western-blotting, cathode buffer with Coomassie Brilliant Blue G-250 was replaced by cathode buffer without Coomassie Brilliant Blue G-250, after the running front had migrated to about two thirds of the separation distance. A HMW calibration kit (GE Healthcare) protein molecular weight markers was used.

Solubilization buffer: $20 \mathrm{mM}$ Tris/ $\mathrm{HCl}(\mathrm{pH} 7.4), 0.1 \mathrm{mM}$ EDTA (pH 8.0), $50 \mathrm{mM} \mathrm{NaCl}, 10 \%$ glycerol, 1 $\mathrm{mM}$ PMSF, supplemented with $1 \%(\mathrm{w} / \mathrm{v})$ digitonin or $0.6 \%(\mathrm{v} / \mathrm{v})$ dodecylmaltoside (DDM) or $0.5 \%(\mathrm{v} / \mathrm{v})$ NP-40.

BN-sample buffer (10x): $100 \mathrm{mM}$ Bis-Tris, $500 \mathrm{mM}$ ع-Amino-n-caproric acid, 5\% (w/v) Coomassie Brilliant Blue G-250, pH 7.0

Acrylamide stock solution: $48 \%$ acrylamide, $1.5 \%$ bisacrylamide (= $49.5 \% \mathrm{~T}, 3 \% \mathrm{C}$ )

$B N$-gel buffer (3x): 200 mM E-amino n-caproic acid, 150 mM Bis-Tris, pH 7.0

$B N$-cathode buffer with Coomassie: $50 \mathrm{mM}$ Tricine, $15 \mathrm{mM}$ bis-Tris, with or without $0.02 \%$ Coomassie Brilliant Blue G-250

$B N$-anode buffer: $50 \mathrm{mM}$ Bis-Tris/HCl, $\mathrm{pH} 7.0$

\subsubsection{Transfer of proteins to PVDF membrane (Western-blotting)}

Proteins separated by SDS-PAGE or BN-PAGE were transferred to PVDF membranes, using semi-dry blotting chambers (Peqlab). PVDF membrane was 
activated in methanol. Prior to the transfer procedure, the activated membrane and Whatman paper were soaked in transfer buffer (20 mM Tris, $150 \mathrm{mM}$ glycine, $0.02 \%$ $(\mathrm{w} / \mathrm{v})$ SDS, $20 \%(\mathrm{v} / \mathrm{v})$ methanol) for few minutes. Electrotransfer of proteins was performed at $250 \mathrm{~mA}$ for $1-2 \mathrm{~h}$.

\subsubsection{Protein detection techniques}

\subsubsection{Coomassie staining}

Coomassie Blue staining was used to stain proteins on PVDF membrane or polyacrylamide gels. The gel or membrane was incubated in Coomassie Brilliant Blue R-250 solution ( $40 \%(\mathrm{v} / \mathrm{v})$ ethanol, $10 \%(\mathrm{v} / \mathrm{v})$ acetic-acid, $0.2 \%$ Coomassie Brilliant Blue R-250). For destaining, a solution containing $40 \%$ ethanol and $10 \%$ $(\mathrm{v} / \mathrm{v})$ acetic acid was used. Before immunodecoration, PVDF membrane was completely destained with methanol and washed with TBS-T buffer (20 mM Tris, 125 $\mathrm{mM} \mathrm{NaCl}, 0.1 \%(\mathrm{v} / \mathrm{v})$ Tween 20, $\mathrm{pH}$ 7.5).

\subsubsection{Gel staining with colloidal Coomassie Brilliant Blue (Neuhoff, 1990)}

Polyacrylamide gels destined for mass spectrometry analysis were incubated in fixer (50\% (v/v) methanol, 2\% (v/v) phosphoric acid) over night. Gels were washed three times in $\mathrm{H}_{2} \mathrm{O}$ for 30 min, followed with an incubation in a solution mixture containing $34 \%(\mathrm{v} / \mathrm{v})$ methanol, $2 \%(\mathrm{v} / \mathrm{v})$ phosphoric acid, $17 \%(\mathrm{w} / \mathrm{v})\left(\mathrm{NH}_{4}\right)_{2} \mathrm{SO}_{4}$, for 30-60 min. Gels were stained with colloidal Coomassie staining solution $(34 \%(\mathrm{v} / \mathrm{v})$ methanol, $2 \%(\mathrm{v} / \mathrm{v})$ phosphoric acid, $17 \%(\mathrm{w} / \mathrm{v})\left(\mathrm{NH}_{4}\right)_{2} \mathrm{SO}_{4}, 0.66 \mathrm{~g} / \mathrm{l}$ Coomassie Brilliant Blue G250) for 2-5 days and then destained by washing in $\mathrm{H}_{2} \mathrm{O}$.

\subsubsection{Immunodetection of proteins on PVDF membranes}

Proteins blotted onto PVDF membrane were visualized by immunodecoration with specific antibodies. After Western blotting, the membrane was incubated in $5 \%(\mathrm{w} / \mathrm{v})$ milk powder in TBS-T (125 mM NaCl, $20 \mathrm{mM}$ Tris, $0.1 \%$ Tween, $\mathrm{pH} 7.5$ ) to block all non-specific binding sites. Incubation was performed for 1 hour at room temperature or over night at $4^{\circ} \mathrm{C}$. The membrane was then incubated with a specific primary 
antibody for 1 hour at room temperature, followed by washing in TBS-T, three times for 15 min. Subsequently, membrane were incubated in an appropriate HRP coupled secondary antibody for 1 hour at room temperature. Membrane were then washed three times for $15 \mathrm{~min}$ in TBS-T, treated with an ECL detection system (GE Healthcare) and the signals were detected on X-ray films (GE Healthcare).

\subsubsection{Detection of radiolabelled proteins by autoradiography}

Dried SDS or BN polyacrylamide gels carrying radioactively labelled proteins were exposed to Storage Phosphor Screens (GE Healthcare), for various time periods, depending on signal intensities (a few hours to a few days). Signals were detected using a Storm820 scanner (GE Healthcare).

\subsubsection{Mass spectrometry and data analysis}

Protein samples were analyzed by mass spectrometry (MS) in the group of $\mathrm{Dr}$. Bettina Warscheid at the Institute for Biology II, University Freiburg (Freiburg). Eluates from IgG-Sepharose affinity purification were analyzed by BN-PAGE (2.6.5) and separated complexes were stained with colloidal Coomassie (2.6.7.2). Visualized complexes were excised from the gel, proteins were acetone precipitated and trypsin digestions were performed in $60 \%(\mathrm{v} / \mathrm{v})$ methanol and $20 \mathrm{mM} \mathrm{NH}_{4} \mathrm{HCO}_{3}$. Peptide mixtures were analyzed by nano-HPLC/ESI-MS/MS as described (Kaller et al., 2011). For peptide and protein identification, mass spectrometric data were searched against a decoy version of the Saccharomyces Genome Database using Mascot (www.matrixscience.com). To assemble proteins based on peptide identification, the ProteinExtractor Tool (version 1.0) in ProteinScape (version 1.3, Bruker Daltonics) was used. Proteins were identified based on at least one unique peptide and a false discovery rate of $5 \%$ was applied.

Eluates from lgG sepharose affinity purifications were also analyzed directly by MS. Spectral counts of proteins identified in six independent experiments were determined and a one-sided Student's t-test was performed. Proteins with a p-value $<0.05$ and sample/control ratio $>5$ were considered as potential interaction partners of the bait protein. Proteins identified by AP-MS experiments were confirmed in at 
least two out of three BN-PAGE analyses.

\subsubsection{In vitro determination of enzyme activities}

Specific activities of the individual complexes were measured spectrophotometrically. All assays were performed with isolated mitochondria.

\subsubsection{Malate dehydrogenase assay}

Malate dehydrogenase activity was assayed by measuring the initial rate of oxaloacetate-dependent NADH oxidation at $340 \mathrm{~nm}$. The measurement was performed in $1 \mathrm{ml}$ scale, using assay buffer $(100 \mathrm{mM}$ potassium phosphate $\mathrm{pH} 7.5$, $0.1 \mathrm{mM} \mathrm{NADH}, 0.2 \mathrm{mM}$ oxaloacetate) and the reaction was initiated by the addition of mitochondria lysate in $0.5 \%(\mathrm{v} / \mathrm{v})$ Triton X-100. The extinction coefficient of NADH at $340 \mathrm{~nm}$ was $6.3 \mathrm{mM}^{-1} \mathrm{~cm}^{-1}$.

\subsubsection{Cytochrome oxidase activity}

To assess the activity of cytochrome oxidase, the oxidation rate of reduced cytochrome c was followed at $550 \mathrm{~nm}$. The assay was performed in $1 \mathrm{ml}$ of $40 \mathrm{mM}$ potassium phosphate $\mathrm{pH} 7.5$, supplemented with $0.02 \%$ reduced cytochrome $c$. Through the addition of Triton X-100 $(0.05 \%(\mathrm{v} / \mathrm{v}))$ mitochondria were solubilized and the reaction was started and followed over time. The reduction of cytochrome $c$ was achieved by the addition of dithionite and excess dithionite was removed by aeration. To determine the concentration of reduced cytochrome $c$, an extinction coefficient of $21.84 \mathrm{mM}^{-1} \mathrm{~cm}^{-1}$ was used.

\subsubsection{NADH-cytochrome $\mathrm{c}$ reductase activity}

The assay was employed to monitor the activity of complex III and was performed at $550 \mathrm{~nm}$ following the increase in absorbance resulting from the reduction of cytochrome $c$. Cytochrome $c$ was resuspended in $1 \mathrm{ml}$ of buffer containing $40 \mathrm{mM}$ potassium phosphate at $\mathrm{pH} 7.5$. As an electron donor to complex III, $0.5 \mathrm{mM} \mathrm{NADH}$ was used and the activity of complex IV was inhibited by the addition $0.1 \mathrm{mM}$ potassium cyanide (Tzagoloff et al., 1975). The reaction was initiated by the addition 
of isolated mitochondria. The concentration of reduced cytochrome $c$ was determined using an extinction coefficient of $21.84 \mathrm{mM}^{-1} \mathrm{~cm}^{-1}$.

\subsubsection{Aconitase activity}

The method to determine aconitase activity was based on the formation of isocitrate from cis-aconitate measured as an absorbance decrease at $240 \mathrm{~nm}$ (Fansler and Lowenstein, 1969). The assay was performed in $500 \mu \mathrm{l}$ scale in quartz cuvette. Prior to data accusation, mitochondria were lysed in $0.5 \%(\mathrm{v} / \mathrm{v})$ Triton X-100 and resuspended in reaction buffer containing $100 \mathrm{mM} \mathrm{NaCl}, 20 \mathrm{mM}$ Tris/ $\mathrm{HCl}$ pH 7.4, 0.1 $\mathrm{mM}$ cis-aconitate. The extinction coefficient of cis-aconitate at $240 \mathrm{~nm}$ was $4.28 \mathrm{mM}^{-}$ ${ }^{1} \mathrm{~cm}^{-1}$.

\subsubsection{Oxygen consumption measurements}

Mitochondrial oxygen consumption was assayed polarographically with a Clark-type electrode (Hansatech Instruments, Norfolk, UK). $100 \mu \mathrm{g}$ of mitochondria were resuspended in $2 \mathrm{ml}$ of assay buffer $(0.3 \mathrm{M}$ sucrose, $10 \mathrm{mM}$ potassium chloride, 5 $\mathrm{mM}$ magnesium chloride, $10 \mathrm{mM}$ potassium phosphate $\mathrm{pH}$ 7.4) After equilibration 2 mM NADH was added as a substrate, which induces oxygen uptake by mitochondria, manifested as a decrease in oxygen concentration in the chamber. Upon the addition of NADH mitochondria were in an ADP limited "state 4" respiration and to induce respiration increase ("state 3") $2 \mathrm{mM}$ ADP was injected into the system, causing a sudden burst in oxygen uptake as the ADP is converted to ATP (Barrientos et al., 2009). Oxygen uptake was monitored until ADP was depleted. Oxygen consumption rates were expressed as $\mathrm{nmol} / \mathrm{ml} / \mathrm{min} / \mathrm{mg}$ of mitochondria fraction protein.

\subsubsection{ROS generation activity}

As an indicator of ROS generation in mitochondria, 2',7'-dichlorodihydrofluorescein diacetate $\left(\mathrm{H}_{2}\right.$ DCFDA) was utilized (Giorgio et al., 2005). $\mathrm{H}_{2} \mathrm{DCFDA}$ is a nonfluorescent molecule, which becomes fluorescent upon oxidation by ROS. 
Mitochondria were incubated in assay buffer $(20 \mathrm{mM}$ Tris/HCl pH 7.4, $150 \mathrm{mM} \mathrm{NaCl}$, $1 \%$ Triton $\mathrm{X}-100$ ), supplemented with $10 \mu \mathrm{M} \mathrm{H}_{2} \mathrm{DCFDA}$. Fluorescence was monitored at excitation and emission wavelengths of $498 \mathrm{~nm}$ and $525 \mathrm{~nm}$, respectively using a HITACHI F-7000 fluorescence spectrophotometer. Data were collected at $0.2 \mathrm{~s}$ intervals over a 10 minute time period.

\subsection{Isolation of mitochondria}

\subsubsection{Isolation of mitochondria from S. cerevisiae}

Mitochondria from S. cerevisiae were isolated as previously described (Meisinger et al., 2006). For the isolation of mitochondria yeast cells were grown to an $\mathrm{OD}_{600}$ of 22.5. Yeast cells were harvested by centrifugation at $7000 \mathrm{~g}$ for $15 \mathrm{~min}$, washed with $\mathrm{H}_{2} \mathrm{O}$ and resuspended in $2 \mathrm{ml} / \mathrm{g}$ of pellet ( $\mathrm{v} / \mathrm{w}$ ) of DTT buffer (10 mM DTT, $100 \mathrm{mM}$ Tris $/ \mathrm{H}_{2} \mathrm{SO}_{4}, \mathrm{pH}$ 9.4). Cells were incubated for $20 \mathrm{~min}$ at $30^{\circ} \mathrm{C}$ with moderate shaking, followed by centrifugation (7000 g, $10 \mathrm{~min}$, room temperature) and washing in $100 \mathrm{ml}$ of zymolyase buffer without enzyme (1.2 M sorbitol, $20 \mathrm{mM}$ potassium phosphate, $\mathrm{pH}$ 7.4). To digest the cell wall and to obtain spheroplasts, the cells were treated with Zymolyase-20T ( $4 \mathrm{mg} / \mathrm{g}$ wet cell pellet) in zymolyase buffer $(7 \mathrm{ml} / \mathrm{g}$ of cells) for 45 min at $30^{\circ} \mathrm{C}$ with moderate shaking. Spheroplasts were isolated by centrifugation (7000 g, $10 \mathrm{~min}$, room temperature) and washed with zymolyase buffer without enzyme. All of the following steps were performed at $4^{\circ} \mathrm{C}$. Washed spheroplasts were resuspended $(7 \mathrm{ml} / \mathrm{g}$ wet cell pellet) in cold homogenization buffer $(0.6 \mathrm{M}$ sorbitol, $10 \mathrm{mM}$ Tris/HCl (pH 7.4), $1 \mathrm{mM}$ EDTA, $0.2 \%(\mathrm{w} / \mathrm{v})$ fatty acid free BSA, $1 \mathrm{mM}$ PMSF). Homogenization was performed by 15 strokes in glass-Teflon homogenizer (Potter S, Sartorius) on ice, followed by two centrifugation steps to remove unopened cells and cell remnants. The first centrifugation step was performed for $5 \mathrm{~min}$ at 2000 $\mathrm{g}, 4^{\circ} \mathrm{C}$ and the second centrifugation for $10 \mathrm{~min}$ at $7000 \mathrm{~g}, 4^{\circ} \mathrm{C}$. To pellet down mitochondria, the supernatant was centrifuged for $15 \mathrm{~min}$ at $17000 \mathrm{~g}, 4^{\circ} \mathrm{C}$. Pelleted mitochondria were washed with SEM buffer (250 mM saccharose, $10 \mathrm{mM}$ MOPS, 1 $\mathrm{mM}$ EDTA, $\mathrm{pH}$ 7.2) and then resuspended in 0.5-1 $\mathrm{ml}$ SEM buffer. Protein concentration was determined by Bradford assay. Mitochondria were diluted to 10 
$\mathrm{mg} / \mathrm{ml}$, aliquoted, frozen in liquid nitrogen and stored at $-80^{\circ} \mathrm{C}$.

\subsubsection{Isolation of mitochondria from human cells}

Mitochondria from HEK-293T cells were isolated as previously described (Lazarou et al., 2009). Cells were harvested, washed with PBS (200 g, $5 \mathrm{~min}$ ) and homogenized in buffer containing $0.1 \% \mathrm{BSA}, 300 \mathrm{mM}$ trehalose, $10 \mathrm{mM}$ HEPES-KOH pH 7.7, 10 mM KCl, 1 mM EDTA, 1 mM EDTA, 0.5 mM PMSF (Yamaguchi et al., 2007) using a glass/Teflon homogenizer (Potter S, Sartorius). Low speed centrifugation (800 g, 10 $\min , 4^{\circ} \mathrm{C}$ ) was used to remove cell debris and nuclei, mitochondria were pelleted at $17000 \mathrm{~g}, 10 \mathrm{~min}, 4^{\circ} \mathrm{C}$. Mitochondria were resuspended in homogenization buffer without BSA.

\subsection{In vitro import analysis in isolated mitochondria}

\subsubsection{In vitro synthesis of radiolabeled mitochondrial proteins}

To label mitochondrial preproteins radioactively, they were synthesized by a separate transcription reaction followed by a translation reaction in the presence of ${ }^{35} \mathrm{~S}$-methionine.

\subsubsection{In vitro transcription}

For in vitro transcription, DNA templates generated by the PCR were used (Ryan et al. 2001). Coding sequences were transcribed into mRNA using an SP6 RNA polymerase. After the purification of the PCR products, in vitro transcription reaction was performed according to the instructions provided by the manufacturer of the RNA polymerase (mMESSAGE mMACHINE SP6 kit, Ambion). The RNA was purified using the MEGAclear kit (Ambion) according to the producer's recommendations.

\subsubsection{In vitro translation}

Labelling of proteins with $\left[{ }^{35} \mathrm{~S}\right]$ methionine was performed by in vitro translation from purified RNA using the Flexi Rabbit Reticulocyte Lysate System (Promega). The 
reaction mixture contained: $66 \mu \mathrm{l}$ rabbit reticulocyte lysate, $2 \mu \mathrm{l}$ translation mix minus methionine, $20 \mathrm{mM}$ DTT, $70 \mathrm{mM} \mathrm{KCl}, 25 \mathrm{mM} \mathrm{MgOAc}, 50 \mu \mathrm{Ci}\left[{ }^{35} \mathrm{~S}\right]$ methionine and 1 $\mu \mathrm{g}$ RNA substrate. The volume was brought to $100 \mu \mathrm{l}$ with RNase-free water. The mixture was incubated at $30^{\circ} \mathrm{C}$ for $1 \mathrm{~h} 30 \mathrm{~min}$ and by addition of cold $20 \mathrm{mM}$ methionine the labelling was stopped. To assess the quality of translated product 1 $\mu \mathrm{l}$ of translation reaction was analyzed by SDS-PAGE and autoradiography. Translation products were frozen in liquid nitrogen and stored in aliquots at $-80^{\circ} \mathrm{C}$.

\subsubsection{In vitro import of radiolabeled preproteins into isolated mitochondria}

In vitro import of radiolabeled precursor proteins into isolated mitochondria was performed as described (Wiedemann et al., 2006; Lazarou et al., 2009).

Single import reactions were performed in a final volume of $100 \mu \mathrm{l}$. Each import reactions contained import buffer, supplemented with $2 \mathrm{mM} \mathrm{NADH}$ and $2 \mathrm{mM}$ ATP. In the case of an extended import reaction in yeast mitochondria, an ATPregenerating system, consisting of $5 \mathrm{mM}$ creatine phosphate and $100 \mu \mathrm{g} / \mathrm{ml}$ creatine kinase, was included. Mitochondria ( $50-75 \mu \mathrm{g}$ per import reaction) were added to the import reaction mixture. The reaction mixture was incubated at $25^{\circ} \mathrm{C}$ for $2 \mathrm{~min}$, to allow the system to equilibrate. Reticulocyte lysate containing radiolabeled precursor protein was added to a final concentration of $2-10 \%(\mathrm{v} / \mathrm{v})$ and incubated for various time periods (1-60 $\mathrm{min}$ ) at $25^{\circ} \mathrm{C}$. Import reactions were stopped by the addition of $1 \%$ AVO mixture (10 $\mu \mathrm{M}$ Antymicin A, $1 \mu \mathrm{M}$ Valinomycin, $20 \mu \mathrm{M}$ Oligomycin) and placing the reaction on ice. Unincorporated protein was degraded by the addition of proteinase $\mathrm{K}(50-100 \mu \mathrm{g} / \mathrm{ml})$. Protease treatment was stopped after $15 \mathrm{~min}$ of incubation on ice by the addition of $2 \mathrm{mM}$ PMSF. After protease treatment, the mixture was centrifuged $\left(20000 \mathrm{~g}, 10 \mathrm{~min}, 4^{\circ} \mathrm{C}\right)$ and the mitochondrial pellets were washed with SEM (250 mM sucrose, $1 \mathrm{mM}$ EDTA, $10 \mathrm{mM}$ MOPS-KOH, pH 7.2) buffer. Mitochondrial pellets were resuspended in $25 \mu \mathrm{l}$ of 1x SDS sample buffer, incubated at $95^{\circ} \mathrm{C}$ for $5 \mathrm{~min}$ and analyzed by SDS-PAGE and autoradiography.

In the case of assaying protein assembly into mitochondria, after the washing step the mitochondrial pellet was solubilized in solubilization buffer (Section 2.6.5) for 15 
minutes on ice. Following clarifying spin $\left(20000 \mathrm{~g}, 10 \mathrm{~min}, 4^{\circ} \mathrm{C}\right)$, the supernatant was mixed with BN-sample buffer (Section 2.6.5) and separated via BN-PAGE.

Import-buffer (yeast): 3\% (w/v) fatty acid-free BSA, $250 \mathrm{mM}$ sucrose, $80 \mathrm{mM}$ potassium chloride, 5 $\mathrm{mM}$ magnesium chloride, $2 \mathrm{mM}$ potassium phosphate, $5 \mathrm{mM}$ methionine, $10 \mathrm{mM} \mathrm{MOPS} / \mathrm{KOH}, \mathrm{pH} 7.2$. Import-buffer (human): 250 mM sucrose, 5 mM MgOAc, 80 mM KOAc, 20 mM Hepes/KOH, pH 7.4, $10 \mathrm{mM}$ sodium succinate, $10 \mathrm{mM}$ malic acid.

\subsection{Localisation and topology of mitochondrial proteins}

\subsubsection{Subcellular localisation analysis by fluorescence microscopy}

Immunofluorescence assay was performed as described (Wurm et al., 2010). In brief, U2-OS cells were grown on cover slips and fixed in $8 \%$ paraformaldehyde (PFA) for 5-10 minutes. Cells were permeablized by a $5 \mathrm{~min}$ incubation in $0.5 \%(\mathrm{v} / \mathrm{v})$ Triton X-100. To detect RCF1a, rabbit antisera against the N-terminus of the protein was used. For the detection of RCF1b antisera directed against the whole protein was utilized. Mitochondrial visualisation was achieved using cyclophilin D. All antibodies were used at a dilution of 1:400. Alexa Fluor 488 conjugated goat antirabbit IgG and Alexa Fluor 594 conjugated goat anti-mouse IgG (Invitrogen) at a dilution of 1:500 were used for detection. Fluorescence microscopy was performed with a beam scanning confocal microscope (TCS SP5, Leica Microsystems CMS $\mathrm{GmbH}$, Wetzlar Germany) equipped with 1.4 NA oil immersion lenses (100 x; HCX PL APO, Leica). The confocal pinhole was set to 1 airy unit. Dual colour images were obtained by sequential scanning. The images are maximum projections of $Z$ stacks. Except for contrast stretching no image processing was applied.

\subsubsection{Submitochondrial localisation of proteins}

Submitochondrial localisation of proteins was determined by protease protection assays as previously described (Frazier et al., 2006) in intact mitochondria and mitoplasts. 


\subsubsection{Preparation of mitoplasts}

To obtain mitoplasts, the outer membrane was ruptured by osmotic swelling in hypotonic solution. Mitochondria in SEM buffer were diluted 1:10 in EM buffer (10 mM MOPS, $1 \mathrm{mM}$ EDTA, pH 7.2) and incubated for $20 \mathrm{~min}$ on ice. As a control, mitochondria were diluted 1:10 in SEM buffer. Mitoplasts were reisolated by centrifugation (20000 g, $10 \mathrm{~min}, 4^{\circ} \mathrm{C}$ ) and resuspended in 1x SDS sample buffer and analyzed by SDS-PAGE and immunodecoration.

\subsubsection{Protease protection assay}

To determine the submitochondrial localisation of proteins, intact mitochondria and mitoplasts were treated with $20 \mu \mathrm{g} / \mathrm{ml}$ and $100 \mu \mathrm{g} / \mathrm{ml}$ of proteinase $\mathrm{K}$ or left untreated. Samples were incubated on ice for $10 \mathrm{~min}$. By the addition of $2 \mathrm{mM} \mathrm{PMSF}$ and incubation for $10 \mathrm{~min}$ on ice, proteinase $\mathrm{K}$ activity was blocked. Mitochondria and mitoplasts were reisolated by centrifugation $\left(20000 \mathrm{~g}, 10 \mathrm{~min}, 4^{\circ} \mathrm{C}\right)$ and resuspended in 1x SDS sample buffer. As a control, $100 \mu \mathrm{g}$ of mitochondria were completely solubilized in EM buffer containing $0.5 \%(\mathrm{v} / \mathrm{v})$ Triton X-100. Solubilized mitochondria were treated with $100 \mu \mathrm{g} / \mathrm{ml}$ proteinase $\mathrm{K}$ or left untreated. In this way all proteins were accessible to proteinase $\mathrm{K}$. The mitochondrial lysate was TCA precipitated and resuspended in 1x SDS sample buffer. All samples were analyzed by SDS-PAGE and immunodecoration with antibodies against proteins from different mitochondrial compartments.

\subsubsection{Carbonate extraction}

Carbonate extraction was employed to analyse the membrane association of a protein. For this purpose, $50 \mu \mathrm{g}$ of mitochondria were centrifuged, resuspended in buffer containing $100 \mathrm{mM} \mathrm{Na}_{2} \mathrm{CO}_{3}, \mathrm{pH} 11.5$ and incubated on ice for $20 \mathrm{~min}$. The samples were then centrifuged $\left(100000 \mathrm{~g}, 45 \mathrm{~min}, 4^{\circ} \mathrm{C}\right.$ ). The soluble proteins from the supernatant were precipitated with TCA, while the pellet containing the membrane associated proteins were directly resuspended in 1x SDS sample buffer. 
The samples were analyzed by SDS-PAGE and immunodecoration of marker proteins.

\subsubsection{Protein precipitation with trichloroacteic acid (TCA)}

Proteins from aqueous solutions were precipitated by adding 50\% TCA to a final concentration of $12 \%(\mathrm{w} / \mathrm{v})$. The samples were incubated for $30-45 \mathrm{~min}$ on ice, and then centrifuged $\left(20000 \mathrm{~g}, 30 \mathrm{~min}, 4^{\circ} \mathrm{C}\right)$. After centrifugation, precipitated proteins were washed with cold acetone $\left(-20^{\circ} \mathrm{C}\right)$, followed with an additional centrifugation step $\left(20000 \mathrm{~g}, 30 \mathrm{~min}, 4^{\circ} \mathrm{C}\right)$. Protein pellet was dried for $10 \mathrm{~min}$ at $\mathrm{RT}$ and resuspended in $1 \mathrm{x}$ sample buffer and incubated at $95^{\circ} \mathrm{C}$ for $5 \mathrm{~min}$.

\subsection{Purification of mitochondrial protein complexes}

\subsubsection{Protein copurification via IgG chromatography}

For protein complex characterization, proteins carrying affinity tags, such as protein A (Graslund at al. 2002) or TAP tag (Puig at al. 2001) were purified via IgG chromatography. For this purpose, mitochondria carrying proteins with C-terminal affinity tag, were resuspended in solubilization buffer $(1 \mathrm{mg} / \mathrm{ml})$ and incubated for 30 $\min$ at $4^{\circ} \mathrm{C}$. The resulting extract was cleared by centrifugation $(20000 \mathrm{~g}, 15 \mathrm{~min}$, $\left.4^{\circ} \mathrm{C}\right)$. IgG beads $(50-150 \mu \mathrm{l})$ were washed twice with $0.5 \mathrm{M}$ acetate buffer $(\mathrm{pH} 3.5)$, and then equilibrated by four washing steps with lysis buffer. The beads and mitochondrial extract were mixed and incubated for $1-2 \mathrm{~h}$ at $4^{\circ} \mathrm{C}$ in an end-over-end shaker. After incubation, the beads were washed extensively with washing buffer. To elute material under native conditions beads with bound protein were treated with TEV protease for 16 hours at $6^{\circ} \mathrm{C}$. In order to remove TEV protease, beads were then incubated with Ni-NTA Sepharose for $30 \mathrm{~min}$ at $4^{\circ} \mathrm{C}$ in an end-over-end shaker and then bound protein was released by centrifugation. Alternatively, bound proteins were released with SDS sample buffer, without $\beta$-mercaptoethanol to prevent the release of IgG chains from the Sepharose. Purified proteins were analyzed by BNPAGE, followed by mass spectrometry or by SDS-PAGE in conjuction with immunodecoration. 
Digitonin-Solubilization buffer: 1\% Digitonin, 10\% (v/v) glycerol, $20 \mathrm{mM}$ Tris/ $\mathrm{HCl} p H$ 7.4, $50 \mathrm{mM} \mathrm{NaCl}$, $1 \mathrm{mM}$ Pefabloc, $1 \mathrm{mM}$ EDTA, $2 \mathrm{mM}$ PMSF, $1 \mu \mathrm{g} / \mathrm{ml}$ Leupeptin

DDM-Solubilization buffer: 0.6\% DDM, 10\% (v/v) glycerol, $20 \mathrm{mM}$ Tris/HCl pH 7.4, $50 \mathrm{mM} \mathrm{NaCl}, 1$ mM Pefabloc, 1 mM EDTA, 2 mM PMSF, 1 $\mathrm{g} / \mathrm{ml}$ Leupeptin

Digitonin-Washing buffer: 0.3\% Digitonin, 10\% (v/v) glycerol, $20 \mathrm{mM}$ Tris/HCl pH 7.4, $50 \mathrm{mM} \mathrm{NaCl}, 1$ mM EDTA, 2 mM PMSF

DDM-Washing buffer: 0.2\% DDM, 10\% (v/v) glycerol, $20 \mathrm{mM}$ Tris/HCl pH 7.4, $50 \mathrm{mM} \mathrm{NaCl}, 1 \mathrm{mM}$ EDTA, 2 mM PMSF

\subsubsection{Co-immunoprecipitation}

Human mitochondrial complexes were immunoprecipitated either with beads coupled to anti-complex IV antibodies or anti-complex $\mathrm{V}$ antibodies. Mitochondria isolated from HEK-293T cells were solubilized in IP-buffer $(20 \mathrm{mM}$ Tris/HCl pH 7.4, $50 \mathrm{mM}$ sodium chloride, $0.5 \mathrm{mM}$ EDTA, 10\% (v/v) glycerol, 1\% (w/v) digitonin, $1 \mathrm{mM} \mathrm{PMSF)}$ at $1 \mathrm{mg} / \mathrm{ml}$. After a clarifying spin $\left(20000 \mathrm{~g}, 15 \mathrm{~min}, 4^{\circ} \mathrm{C}\right)$, the supernatant (total) was incubated with antibody- coupled beads for 1 hour at $4^{\circ} \mathrm{C}$. Samples were extensively washed with W-buffer (IP-buffer with $0.3 \%(\mathrm{w} / \mathrm{v})$ digitonin). Elution of bound proteins was performed with $1 \times$ SDS-sample buffer. Isolated proteins were analyzed by SDS-PAGE followed by Western-blotting and immunodecoration. 


\section{Results}

\subsection{Identification of Rcf1 and Rcf2}

\subsubsection{Purification and analyses of respiratory chain supercomplexes}

Respiratory chain complexes III and IV in Saccharomyces cerevisiae assemble into supercomplexes, which are comprised of a complex III dimer in complex with one or two copies of complex IV. These complexes can be separated and analyzed by BNPAGE (Schägger and Pfeiffer, 2000). Despite the fact that the existence of supercomplexes has been long established and verified by a variety of different approaches, their molecular composition is still poorly understood. Moreover, the organization of supercomplexes is not limited to the aforementioned forms, but rather multiple forms of these supercomplexes have been identified in mitochondria, differing in regards to their interaction partners. It has been shown that the TIM23 protein translocase (van der Laan et al., 2006; Saddar et al., 2008) and components of its associated import motor, the PAM machinery (Wiedemann et al., 2007), can associate with supercomplexes. Respiratory chain assembly factors (Mick et al., 2007) and translational regulators (Mick et al., 2010) have been found to interact with a subpopulation of supercomplexes, as well as the ADP/ATP carrier (Dienhart and Stuart, 2008), enzymes involved in lipid modification (Claypool et al., 2008) and fatty acid $\beta$ oxidation (Wang et al., 2010).

To analyze the composition of yeast $S$. cerevisiae respiratory supercomplexes and identify novel components, supercomplexes containing complex III/IV were isolated from mitochondria. Therefore, a functional TAP-tagged version of the complex III subunit Cor1 was used (van der Laan et al., 2006; Wiedemann et al., 2007). Upon digitonin solubilization of mitochondria, complexes were purified by IgG chromatography and released from the column by TEV-protease treatment. Isolated complexes were separated by BN-PAGE (Figure 3.1A). As a control, the corresponding wild type strain lacking a tag on Cor1 was treated in the same manner. The isolation of respiratory chain supercomplexes $\mathrm{III}_{2}$, $\mathrm{II}_{2} / \mathrm{IV}$ and $\mathrm{III}_{2} / \mathrm{IV}_{2}$ was validated through Western-blotting (Figure 3.1B). After colloidal coomassie staining 
of the gel, visualized protein complexes were excised and subjected to in-gel digestion allowing for the resulting peptides to be analyzed by mass spectrometry. Known subunits of complex III and complex IV as well as a subset of known associated proteins were identified. Additionally, the following proteins were detected: Rcf1 (YML030W), Rcf2 (YNR018W), Aim10 (YER087W), Aim24 (YJR080C), Mio10 (YCL057C-A), Fmp25 (YLR077W), Cox26 (YDR119W-A). At this time none of these proteins had been functionally characterized, although Rcf1 and Rcf2 (also known as Aim31 and Aim38, respectively) as well as Aim10 and Aim24 had been identified in a genome wide screen as genes involved in mitochondrial inheritance (Hess et al., 2009) (Figure 3.1B).

A

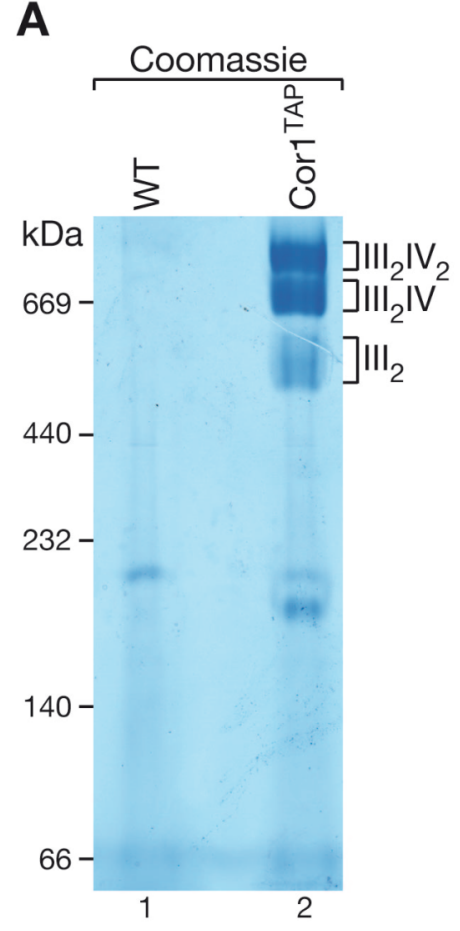

B

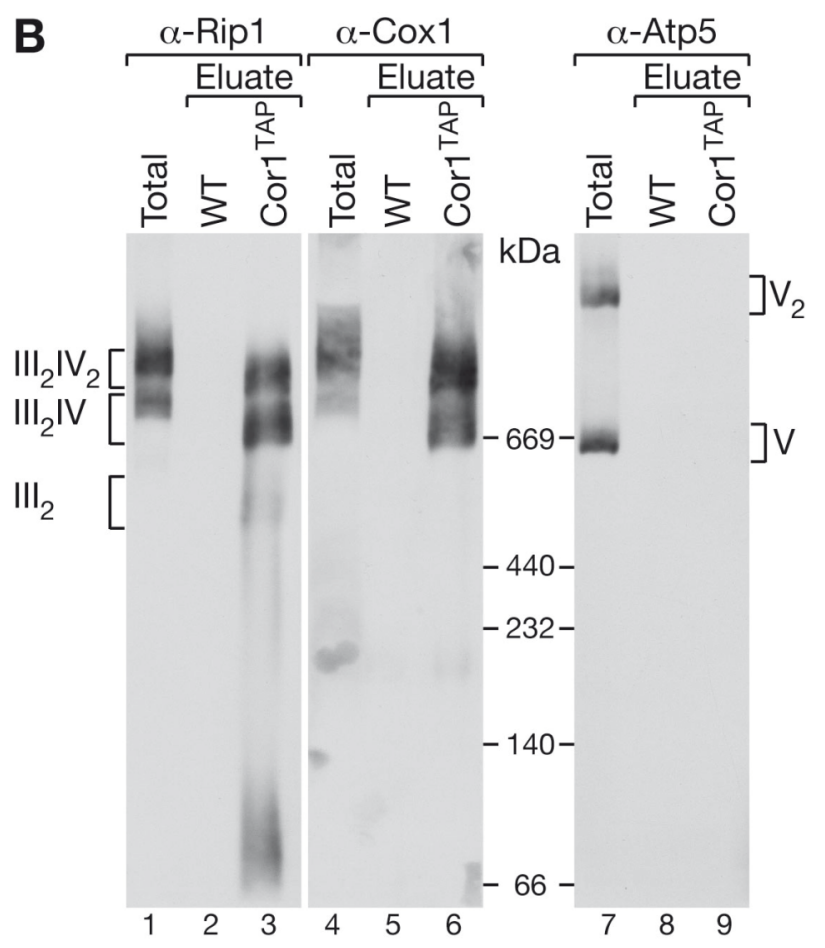

Figure 3. 1 Purification of Respiratory Chain Supercomplexes. WT mitochondria and mitochondria containing TAP tagged Cor1 were solubilized in digitonin-containing buffer and subjected to IgG chromatography. Bound proteins were released by TEV protease cleavage. Eluates were analyzed by BN-PAGE, and complexes were visualised by commassie brilliant blue staining (A) or by Western-blotting (B). The supercomplexes in Cor1TAP lanes are shifted in size as compared to the totals due to the low protein amount in purified complexes. Total, $10 \%$; eluate $100 \%$. Protein complexes from the gel in panel $A$ were analyzed by mass spectrometry. 
To assure specificity of the observed interaction partners, the isolation was performed three times. In addition, six independent supercomplex isolations were performed and the elution fractions were directly processed via mass spectrometry.

\begin{tabular}{|c|c|c|c|}
\hline $\begin{array}{l}\text { Subunits of } \\
\text { cytochrome } c \\
\text { reductase }\left(\mathrm{bc}_{1}\right)\end{array}$ & $\begin{array}{l}\text { Subunits of } \\
\text { cytochrome } \\
\text { oxidase (COX) }\end{array}$ & $\begin{array}{l}\text { Assembly factors } \\
\text { and associated } \\
\text { proteins }\end{array}$ & Novel proteins \\
\hline $\begin{array}{l}\text { COR1/YBL045C } \\
\text { QCR2/YPR191W } \\
\text { QCR6/YFR033C } \\
\text { QCR7/YDR529C } \\
\text { QCR8/YJL166W } \\
\text { QCR9/YGR183C } \\
\text { RIP1/YEL024W } \\
\text { COB/Q0105 } \\
\text { CYT1/YOR065W }\end{array}$ & $\begin{array}{l}\text { COX1/Q0045 } \\
\text { COX2/Q0250 } \\
\text { COX3/Q0275* } \\
\text { COX4/YGL187C } \\
\text { coX5a/YNL052W } \\
\text { COX5b/YIL111W } \\
\text { COX6/YHR051W } \\
\text { coX7/YMR256C } \\
\text { coX8/YLR395C } \\
\text { coX9/YDL067C } \\
\text { COX12/YLR038C } \\
\text { coX13/YGL191W }\end{array}$ & $\begin{array}{l}\text { CBP3/YPL215W } \\
\text { CBP4/YGR174C } \\
\text { SHY1/YGR112W } \\
\text { COX11/YPL132W } \\
\text { COX14/YML129C } \\
\text { COX16/YJL003W } \\
\text { TIM21/YGR033C } \\
\text { MDM38/YOL027 } \\
\text { C } \\
\text { AAC2/YBL030C } \\
\text { PET54/YGR222W }\end{array}$ & $\begin{array}{l}\text { RCF1/YML030W } \\
\text { RCF2/YNR018W } \\
\text { FMP25/YLR077W } \\
\text { COX26/YDR119W-A }\end{array}$ \\
\hline
\end{tabular}

For proteins identified in these experiments spectral counts were determined and one-sided Student's t-test was performed. Proteins with a p-value $<0.05$ and sample/control ratio $>5$ were considered as potential interaction partners of Cor1 protein. Additionally these proteins had to be found in two out of three BN-PAGE analyses, which allowed for a list of proteins considered to specifically be co-purified with supercomplexes to be compiled (Table 3.1).

\subsubsection{Assaying precursor import of candidate proteins}

Although Aim10 and Aim24 were not identified in all isolation repetitions as well as Mio10 not meeting all requirements, the first experiments concerning mitochondrial localization were performed not just with potential interaction partners listed in table 3.1, but also with these three proteins. Computer analyses (MITOPROT) of the primary structure of all seven proteins showed that all identified proteins, except 
Rcf1, Rcf2 and Mio10, possess a presequence and have a high probability of mitochondrial localization (Table 3.2).

\begin{tabular}{lcc}
\hline \multicolumn{2}{c}{ Table 3.2 Prediction of mitochondrial targeting for candidate proteins } \\
\hline Protein & $\begin{array}{l}\text { Presequence/ } \\
\text { Cleavage site }\end{array}$ & $\begin{array}{c}\text { Probability of import } \\
\text { into mitochondria (\%) }\end{array}$ \\
\hline Rcf1 & Not predicted & 26.44 \\
Rcf2 & Not predicted & 3.43 \\
Aim10 & Predicted/15 & 82.14 \\
Aim24 & Predicted/23 & 96.86 \\
Mio10 & Not predicted & 0.69 \\
Cox26 & Predicted/20 & 97.2 \\
Fmp25 & Predicted/26 & 97.12 \\
\hline
\end{tabular}

All proteins were synthesized in vitro in the presence of $\left[{ }^{35} \mathrm{~S}\right]$ methionine and import experiments using isolated yeast mitochondria were performed. Mio10 and Cox26 failed to import, as well as Fmp25, while Aim10, Aim24, Rcf1 and Rcf2 were imported efficiently. In the presence of a membrane potential, all four proteins were transported to a protease protected compartment (Figure 3.2), inferring their translocation into mitochondria. In addition, Aim24 was processed to a faster migrating form (Figure $3.2 \mathrm{C}$ ), consistent with a predicted cleavage site for this protein, unlike Aim10 for which the cleavage is predicted but processing was not observed (Figure 3.3D). These initial experiments designated Aim10, Aim24, Rcf1 and Rcf2 as potential interaction partners of respiratory chain supercomplexes. 
A

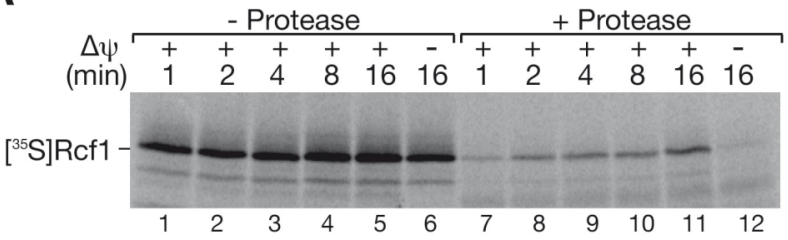

B

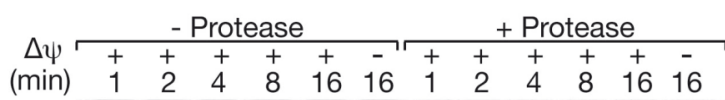

${ }^{35}$ S]Rcf2-
C

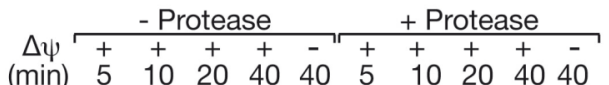

[55]Aim24

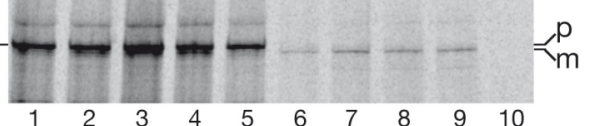

D

$\Delta \psi+$ - Protease + Protease

(min) $\begin{array}{cccccc}10 & 20 & 20 & 10 & 20 & 20\end{array}$

[5S]Aim10-

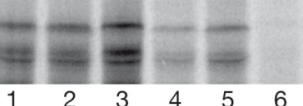

Figure 3. 2 Import of candidate proteins depends on membrane potential. Radiolabelled precursor proteins of Rcf1 (A), Rcf2 (B), Aim24 (C) and Aim10 (D) were incubated with isolated WT mitochondria for the indicated times. After import samples were protease treated where indicated and analyzed by SDS-PAGE and digital autoradiography.

Despite the fact that Cox26, Fmp25 and Mio10 did not import into mitochondria in vitro, each of them was efficiently identified in all isolation replicates. Later on the work of other groups described Cox26 as a protein that can bind to the cytochrome oxidase subunit, Cox2 (Wumaier et al., unpublished data), and Fmp25 as a novel assembly factor of complex III (Mathieu et al., 2011), while Mio10 is still considered a potential supercomplex interaction partner.

\subsubsection{Localization and topology of Rcf1, Rcf2 and Mio10}

To analyze the localization of Mio10, and to confirm mitochondrial localization of Rcf1 and Rcf2, specific antibodies against these proteins were raised. Antibodies were directed against the C-termini of the proteins and could efficiently recognize the indicated proteins in purified yeast mitochondria (Figure 3.3). Deduced primary sequence predicted that Rcf1, Rcf2 and Mio10 contain two transmembrane (TM) domains. When mitochondria were subjected to carbonate extraction at $\mathrm{pH} 11.5$, Mio10, Rcf1 and Rcf2 remained in the pellet fraction, typical of integral membrane proteins such as Tim21, while peripheral membrane proteins as Tim44 were extracted (Figure 3.3A and 3.3B). 

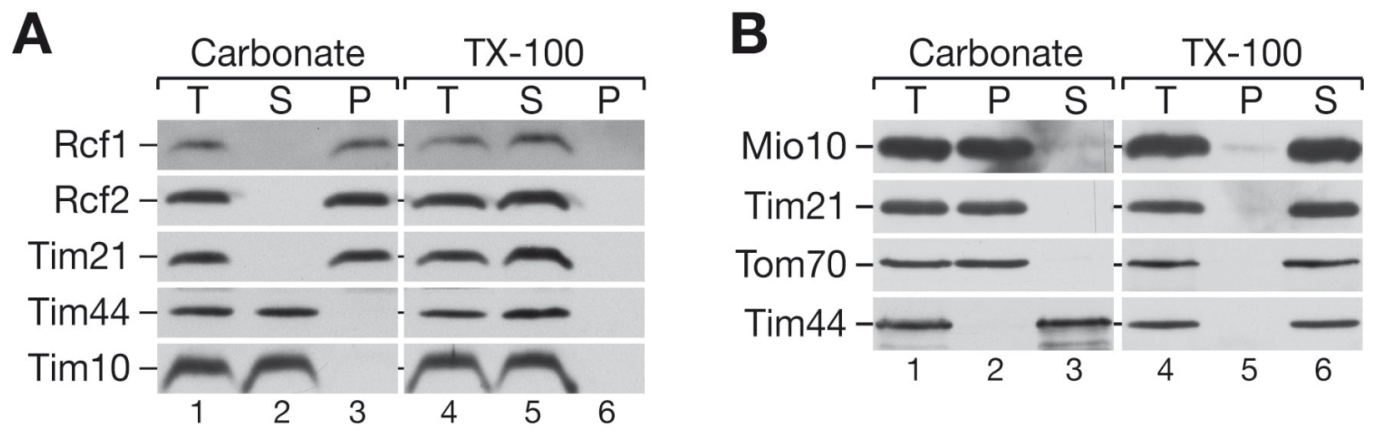

Figure 3. 3 Rcf1, Rcf2 and Mio10 are membrane proteins. (A), (B) Mitochondria isolated from yeast cells were carbonate extracted at $\mathrm{pH} 11.5$ or lysed with Triton $\mathrm{X}-100$. Samples were either left untreated $(T$, total) or separated into pellet $(P)$ and supernatant (S) by centrifuging at $100000 \mathrm{~g}$, and subjected to SDS-PAGE and Western-blotting.

To analyze further submitochondrial localization of these proteins, protease protection assays were performed. In intact mitochondria all three proteins were unaffected, whereas the outer membrane protein Tom70 was protease sensitive (Figure 3.4A and 3.4B). However, when the outer mitochondrial membrane was disrupted by osmotic swelling (mitoplasts), these proteins became accessible to the protease (Figure 3.4A and 3.4B), indicating that the C-termini of Mio10, Rcf1 and Rcf2 are exposed to the intermembrane space. No stable proteolytic fragments of Mio10, Rcf1 or Rcf2 were detected (Figure 3.4C). The inner membrane of the mitoplasts remained intact, as the matrix protein Tim44 was resistant to protease treatment and became accessible only after disruption of the inner membrane by Triton X-100 (Figure 3.3A and 3.3B). Taken together, Mio10, Rcf1 and Rcf2 are integral proteins of the inner mitochondrial membrane with their C-terminal domains exposed to the intermembrane space and prediction of two transmembrane segments suggests that their $\mathrm{N}$-termini also localize to the intermembrane space. 

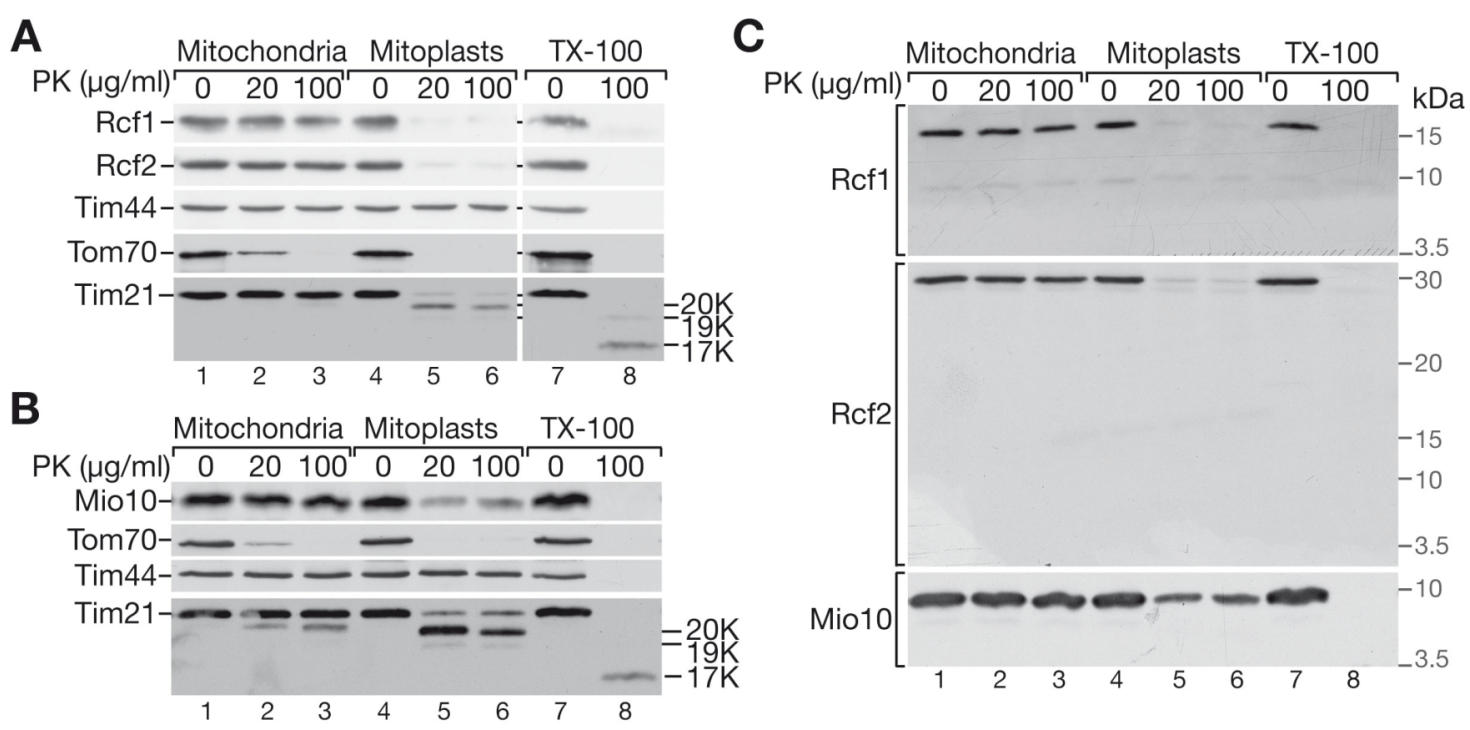

Figure 3. 4 Rcf1, Rcf2 and Mio10 are mitochondrial inner membrane proteins. (A), (B), (C) Isolated mitochondria were left untreated, swollen under hypotonic conditions (mitoplasts) or lysed with Triton X-100, followed by proteinase $\mathrm{K}$ treatment at indicted concentrations. Samples were analyzed by SDS-PAGE and immunodecoration. (C) Presented are sections of the blots below Rcf1, Rcf2 and Mio10. No proteolytic fragments of Rcf1, Rcf2 and Mio10 were detected with antibodies directed against the C-termini.

Although by this approach Mio10 was confirmed to be a mitochondrial protein, it was not further investigated in this work. Further characterization on the function of Mio10 within mitochondria was reported by Alkhaja et. al. 2012, where it was described as a novel constituent of Fcj1 complexes responsible for mitochondrial architecture.

\subsubsection{Rcf1 and Rcf2 associate to respiratory chain supercomplexes}

Initial experiments revealed efficient import of Aim10, Aim24, Rcf1 and Rcf2 into mitochondria and as mentioned above, they were considered to be potential interaction partners of respiratory chain supercomplexes in digitonin solubilized mitochondrial membranes. To test this, after import mitochondria were solubilized in digitonin containing buffer and the resulting complexes separated by BN-PAGE. Rcf1 and Rcf2 assembled mainly into two high molecular weight protein complexes and the migration patterns were reminiscent of respiratory chain supercomplexes (Figure 3.5). Comigration of these two proteins with supercomplexes was also suggested in proteomic analyses of crude mitochondrial extracts separated by BN-PAGE (Helbig 
et al., 2009). In contrast, Aim10 and Aim24 did not assemble into these complexes, but to complexes of intermediate size (Figure 3.5). As stated above, the main goal of this project was to identify novel factors associated with supercomplexes, so further characterization of Aim10 and Aim24 will not be further discussed here, and the main focus of this work was to more precisely define Rcf1 and Rcf2 (Respiratory Supercomplex Factor 1/2).

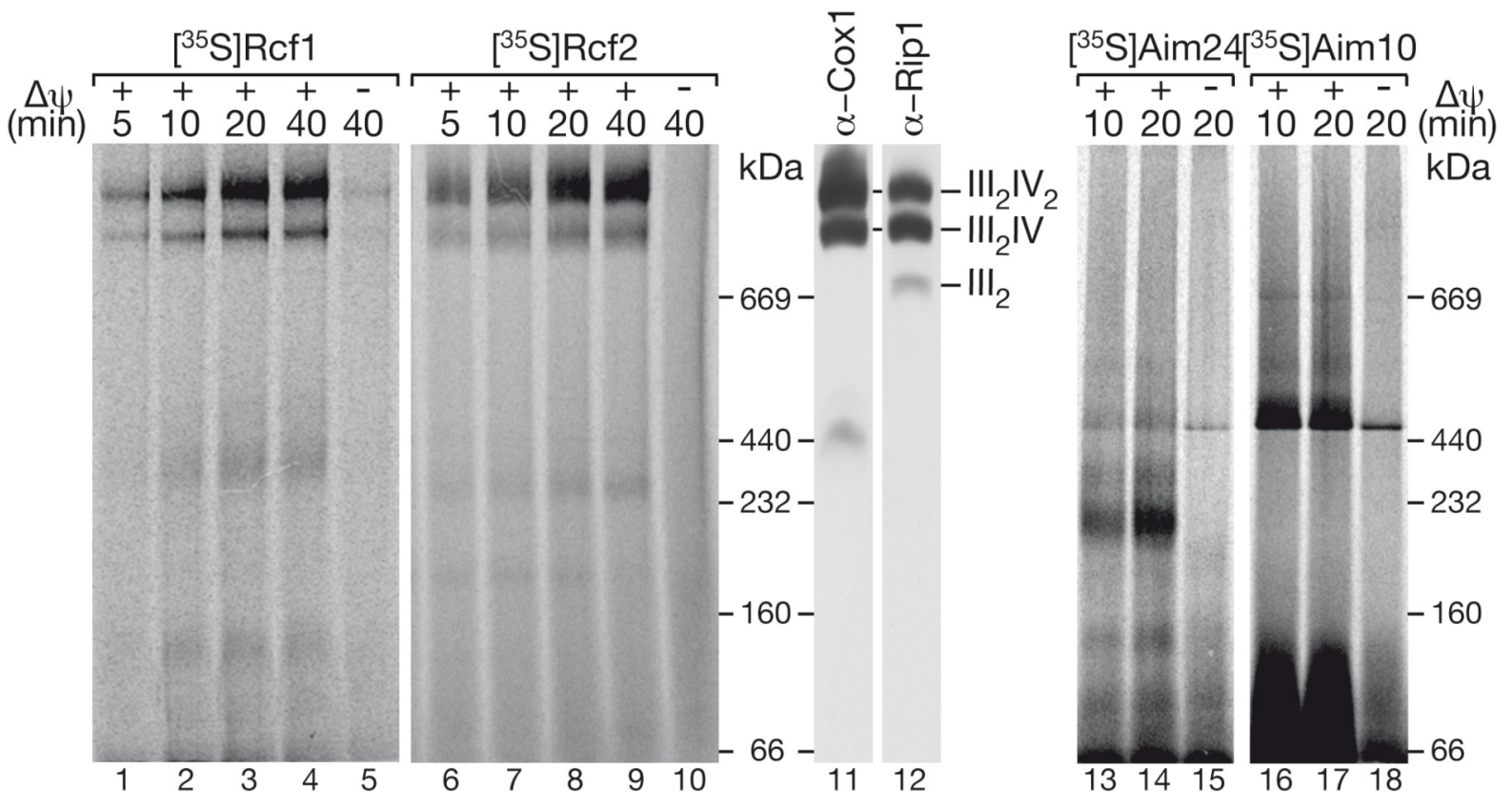

Figure 3. 5 Rcf1 and Rcf2 associate with respiratory chain supercomplexes. Radiolabelled Rcf1, Rcf2, Aim24 and Aim10 were imported into isolated mitochondria in the presence or absence of membrane potential $(\Delta \psi)$, for indicated times. Upon import samples were treated with proteinase $\mathrm{K}$ and solubilized in digitonin buffer. Samples were analyzed by BN-PAGE and digital autoradiography. For comparison, mitochondria were solubilized and analyzed by BN-PAGE and Western-blotting.

To confirm the mass spectrometric results and to obtain further evidence of the initial observation that Rcf1 and Rcf2 can associate with respiratory chain supercomplexes, purified supercomplexes were analyzed by SDS-PAGE and Western-blotting. Rcf1 and Rcf2 were copurified with Cor1 ${ }^{\text {TAP }}$, as well as the known structural subunits of the respiratory chain (Figure 3.6). 

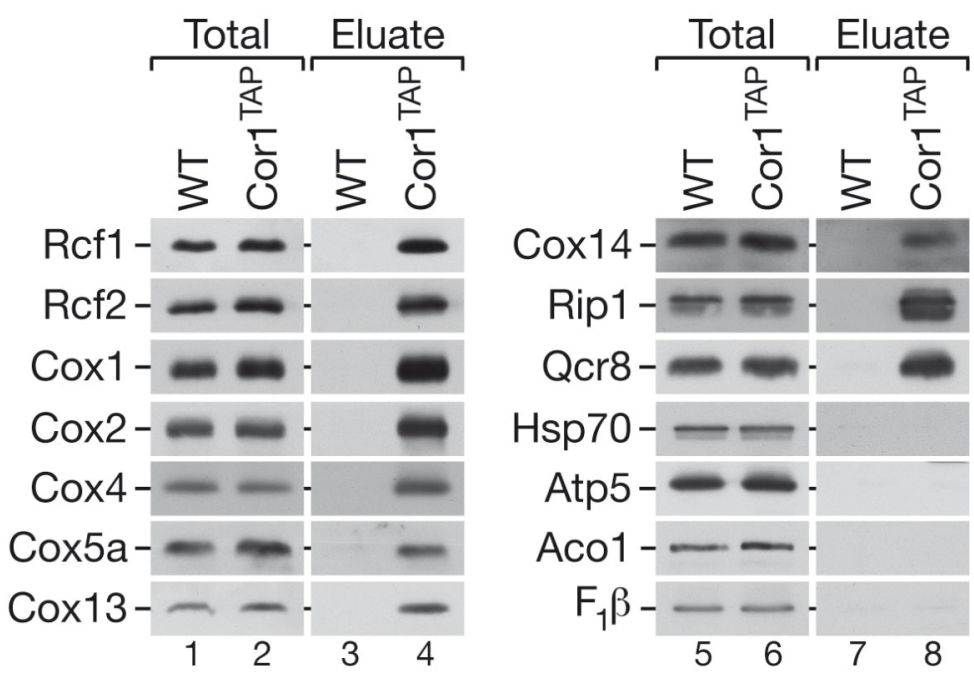

Figure 3. 6 Rcf1 and Rcf2 co-isolate with purified respiratory chain supercomplexes. Mitochondria isolated from wild type (WT) and cor $1^{\text {TAP }}$ strains were solubilized in digitonin-containing buffer and subjected to IgG chromatography. Bound proteins were released by TEV protease cleavage. Eluates were analyzed by SDSPAGE, Western-blotting and immunodecoration. Total $10 \%$; eluate $100 \%$.

\subsubsection{Rcf1 and Rcf2 are associated with the cytochrome oxidase}

When the migration patterns of Rcf1 and Rcf2 were compared to that of the respiratory chain supercomplexes on BN-PAGE, they were similar to that of complex IV and no complexes that correspond to the size of complex III dimers were observed (Figure 3.5). This data indicated the association of Rcf1 and Rcf2 to complex IV and to support this finding, both proteins were imported into mutant mitochondria. $\operatorname{cox} 4 \Delta$ mitochondria lack Cox4 a structural subunit of complex IV, and therefore cannot form mature complex IV, while cyt1s do not contain mature complex III (Figure 3.7). Formation of the supercomplexes in these strains is abolished, but absence of one complex does not influence formation of the other. This explains the fact that in the cox $4 \Delta$ strain complex III is present exclusively as a dimer and in the cyt1 $1 \Delta$ strain complex IV is found as two complexes between 230 and $440 \mathrm{kDa}$ (Figure 3.7). BN-PAGE analyses of imported Rcf1 and Rcf2 revealed a migration pattern similar to that of Cox4 and Cox13 in cyt1 $1 \Delta$ mitochondria, while the assembly of both proteins into higher molecular weight complexes was blocked in 
$\operatorname{cox} 4 \Delta$ mitochondria (Figure 3.7). Therefore, Rcf1 and Rcf2 are components of complex IV and supercomplexes in yeast mitochondria.

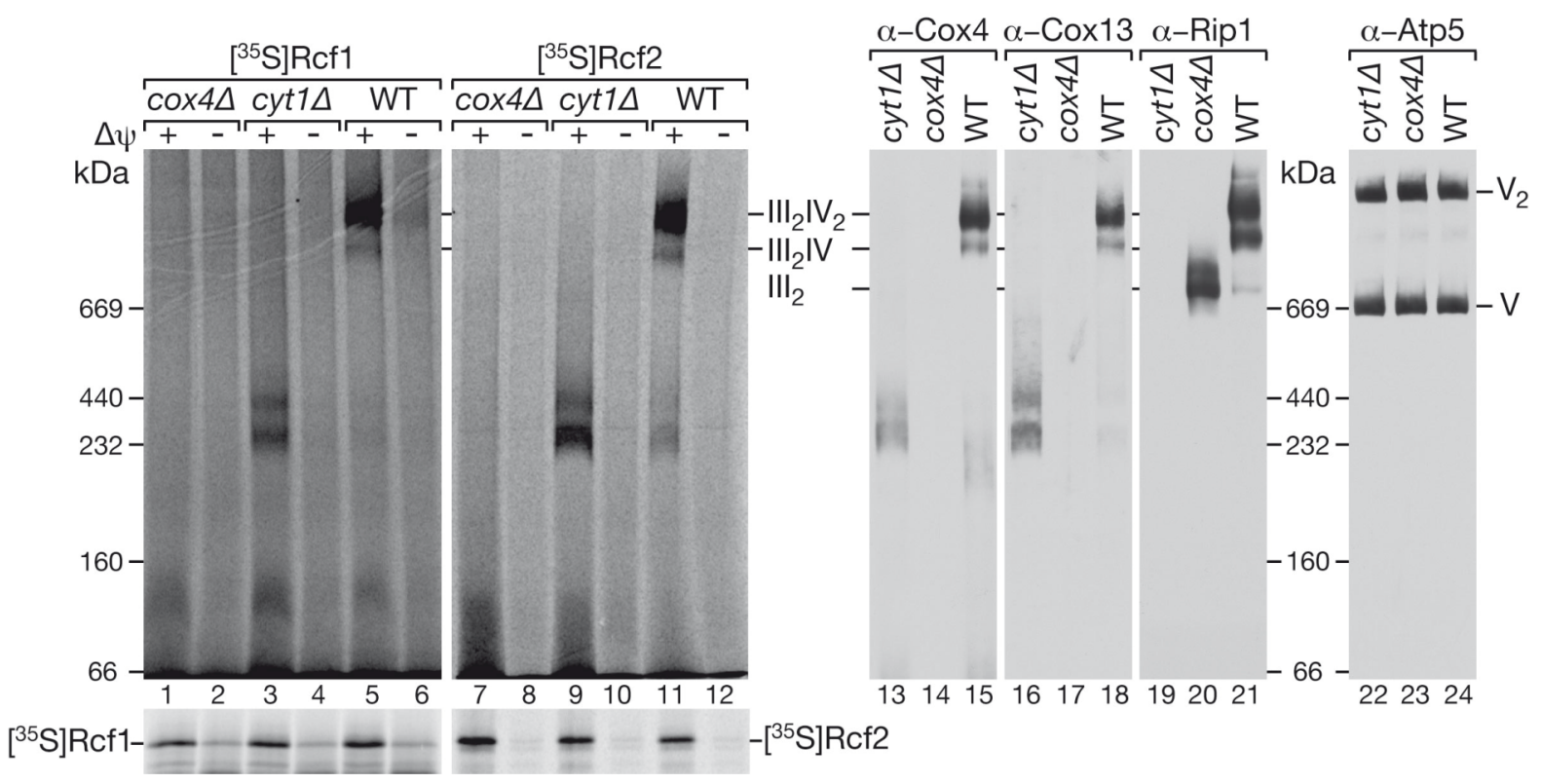

Figure 3. 7 Rcf1 and Rcf2 associate to cytochrome oxidase. Radiolabelled Rcf1 and Rcf2 were imported into mitochondria isolated from WT, cox $4 \Delta$ and cyt1 $\Delta$ strains, in the presence or absence of $\Delta \psi$, treated with proteinase $\mathrm{K}$ and solubilized in digitonin buffer. Samples were analyzed by BN-PAGE and digital autoradiography. For comparison, mitochondria isolated from indicated strains were solubilized in digitonin buffer and analyzed by BN-PAGE and Western-blotting.

\subsubsection{Rcf1 is conserved among eukaryotes}

The deduced amino acid sequences predict proteins of 159 amino acid residues for Rcf1 and 224 for Rcf2. Rcf2 is conserved among yeast species (Figure 3.8C), whereas Rcf1 possess homologs from yeast to higher eukaryotes, including plants and human (Figure 3.8A and 3.8B), with two homologs in human (RCF1a (Q9Y2412) and RCF1b (Q9BW72)) and mouse. 
$\mathbf{A}_{\mathrm{s}, a t}$

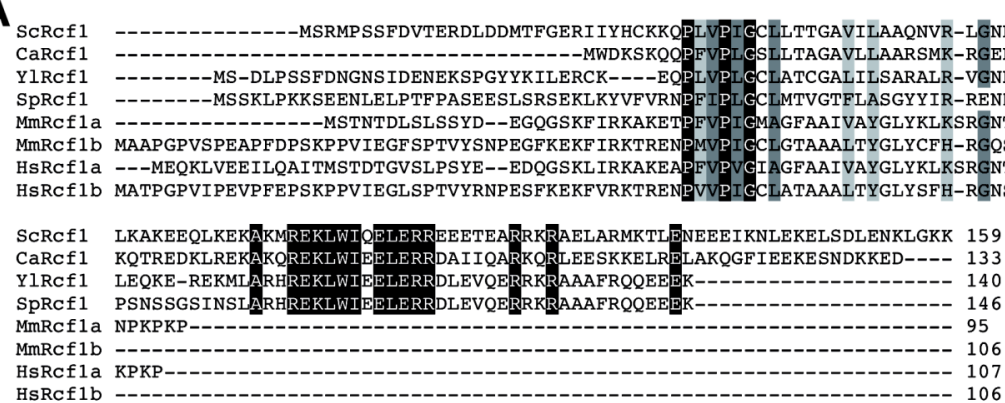

B

ScRCf1 ------MSRMPSSFDVTERDLDDMTFGERI IY HCKKOPLVPIGCLITTGAVILAAQNVRLGNKWKAQYYFRWRVGLQAATLVALVAGSFIYGTSGKE------------ 91 AtRcf1 -------MSSVEPD---MEDLFQE-KKRVRN---PLVPL GALMTAGVLTAGLISFRRGNSQLGOVLMRARVVVOGATVALMVGTGYYYGDNPWKKLLLSEIHETEA 92 GmRCf1 ---------MA---------EDYLQEE KKRVRN---PFVP IGALVIAGVLTAGLISFRQGNSQLGQKLMRARVVVQGATVALMVGTAFYYGENPWR------------ 75

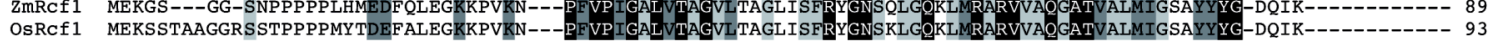

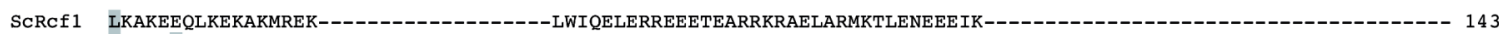
AtRCf 1 LSPKSSSAATLTLMNQKDPSSSSIVSVLCLVISGLALIIVFLGVLYLIFKFLRKSSTLFPIPHFNYNPDLFSFSSPQLQHLFFLHDSGLDQTAIDALPVFLYGNVTISL 201

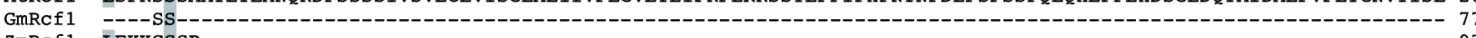

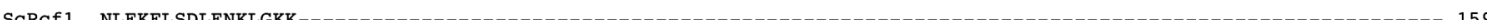
AtRC 1 EQPFDCAVCLNEFSDTDKLRLLPVCSHAFHLHCIDTWLLSNSTCPLCRRSLSTSNVCYNHSETLVAPLSGHQQVDDGKASLAKRVFSVRLGRFKSTNESQSQRHDVKDE 310

SCRCf1 -----------159 15

ATRCA 1 IGVRMPRRCYSMGTQQYLVCDQDFVVALSSSPREGNIGR 349

GRRf1 ------- 77

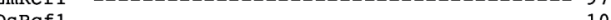

C

SCRCf2 MKILTQDEIEAHRSHTLKGGIEGALAGFAISAIIFKVLPRRYPKFKPSTLTWSIKTALWITPPTVLTAICAEEAASNNFDATMYGSGSSSEDALDEHRRKSLSTKDKFVE 110 CaRCf2 MKILSKEEKDAHISHI ISEGAKGLFYGGAL SVGLYQYLKHRHP-ARFATFNPSIKAAIIAMPT ISVAAFFADQGSVEFDRKMHQSEYQEAKYLEEYREWNRLSLSDKCFT 109

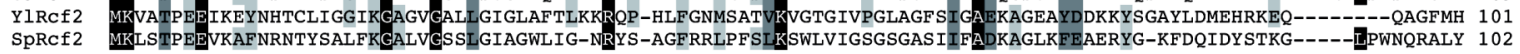

ScRCf2 GLSNNKYKKIITGAWAASLYGSWVIVNKDPIMTKAQKI VQARMYAQFITVGLITASVGLSMYE------------------NKLHPNKQKVNEMR---RẄENALRVAEEEE 199 CaRCf2 VLNDNKYKII ISAWAGSL YGSWVLVNRDKIMDTAOKAVQARMYAOGITIILLI GTLILAMKE-------------EEIN--KKKPKPIP---E WKRI IMEKEAED 196 Y1RCf2 YLKEHKYKCVFGGWVVSMAGSMWYVFRDKYMTSTOKIVOARMYAQASTIILLFATLAFSASDNLGGGNNSRDFLG---KDGKVHHHHVQLEHSD---DWKELVKEEEDRL 206 SPRCf 2 YFNEHKWIIIGTWASTMGLSLYASRNRYDTAPQKLIQARMYAOGVTVVVLLSVYLSTLANRLEPLEREVLVTDPSNPTKLVAFKQRKERYPGELQWEVLVSQDEERL 212

ScRcf2 -RLEKEGRRT----------------------------------GYVSNEERINSKIFKS-------- 224

Y1RCf2 KEAKAEAKQHSQELKAEVKSTADEIKEKTKGKFDEAKKSAADAAEDAADKAEDVADDAKEKAEEYKEDVKKAVK 279

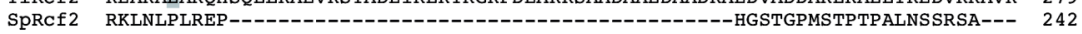

Figure 3. 8 Rcf1 is conserved among eukaryotes, while Rcf2 is conserved among fungi. Alignment of Rcf1 homologs and Rcf2 homologs with ClustalW (2.0.12). Black boxes, identical residues in all species, grey boxes similar amino acids. (A) Alignment of Rcf1 with fungal and animal homologs. Sc S. cerevisiae; Ca C. albicans; YI Y. lipolytica; Sp S. pombe; Mm M. musculus; Hs H. sapiens. (B) Alignment of Rcf1 with plants homologs. Sc S. cerevisiae; A. thaliana; G. max; Z. mays; O. sativa.. (C) Alignment of Rcf2. Sc S. cerevisiae; Ca C. albicans; YI Y. lipolytica; Sp S. pombe.

In cooperation with the group of Prof. Dr. Katschinski, Department of Cardiovascular Physiology, University Göttingen, expression of RCF1a and RCF1b in different murine tissues was assessed using quantitative Real-Time-PCR. The analyses revealed that RCF1a and $b$ were predominantly expressed in metabolically active tissues, such as the heart, liver and kidney (Figure 3.9). 

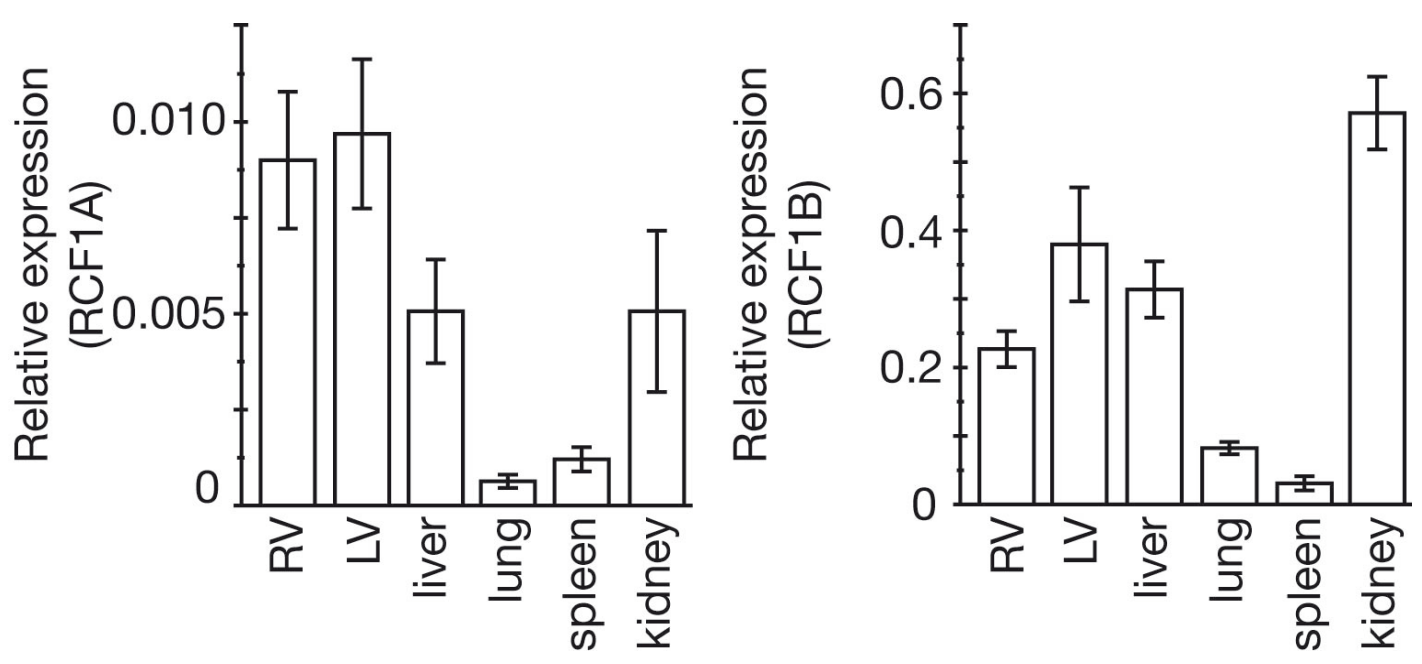

Figure 3. 9 Expression profiles of RCF1a and RCF1b in different mouse tissues. mRNA expression of RCF1A and RCF1B in indicated mouse tissue samples expressed as a relative expression to the housekeeping gene $\mathrm{mS} 12\left(2^{-\Delta \mathrm{Ct}}\right)$. Right ventricle (RV), Left ventricle (LV).

\subsubsection{Localization and topology of RCF1a and RCF1b}

To test if RCF1a and $b$ are mitochondrial proteins in mammals, immunofluorescence analyses were performed. These analyses were carried out in the lab of Prof. Dr. Stefan Jakobs, Department of NanoBiophotonics, Mitochondrial Structure and Dynamics Group, Max-Planck Institute for Biophysical Chemistry, Göttingen. Proteins were labeled with specific antibodies directed against N-terminus of RCF1a and against the whole RCF1b protein. Immunofluorescence analyses showed that the labeling pattern of both proteins could be superimposed with mitochondria visualized by cyclophilin D (Figure 3.10), confirming their localization within mitochondria. 

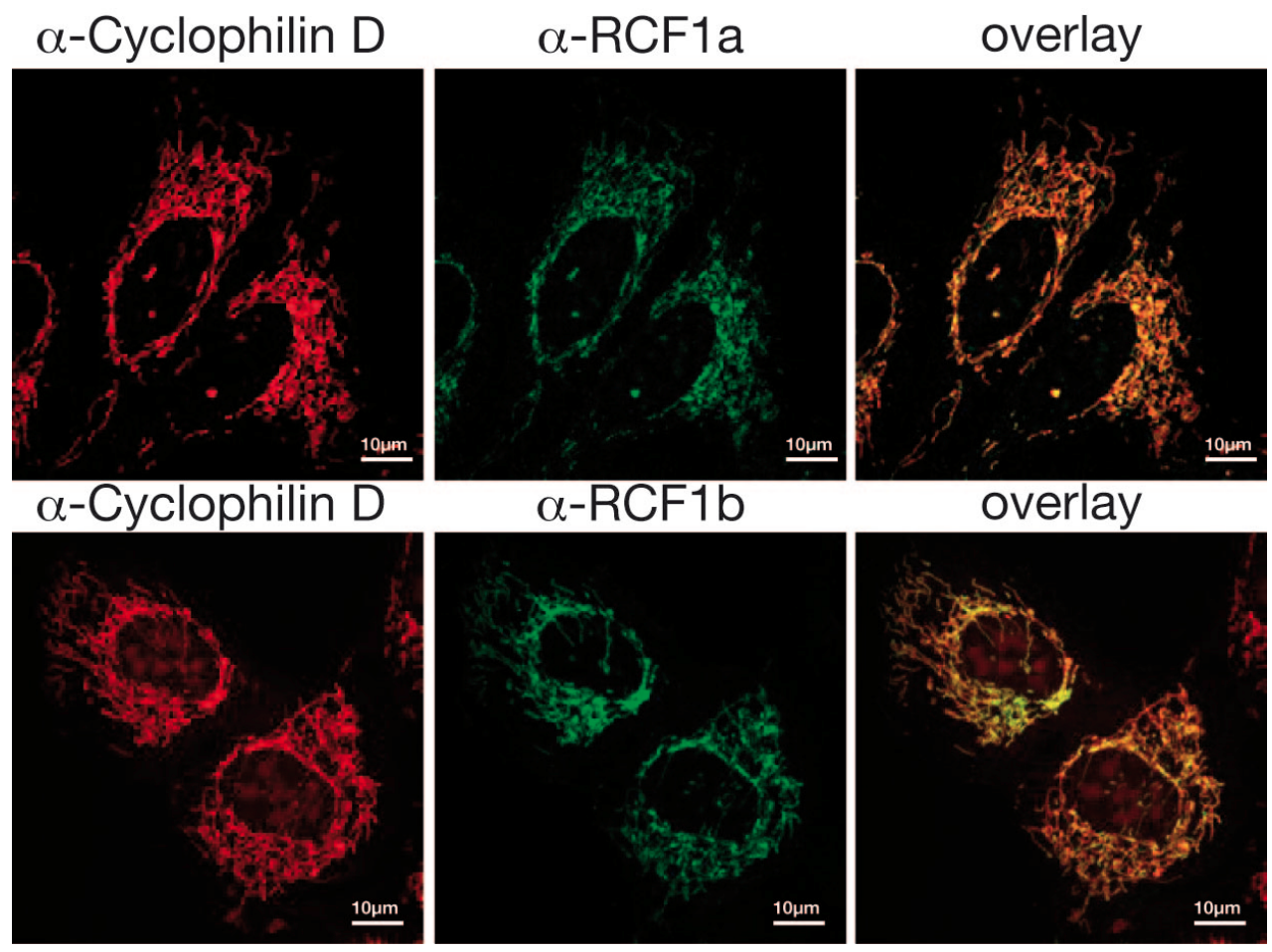

Figure 3. 10 RCF1a and RCF1b, are localised within mitochondria. Subcellular localization of RCF1a and RCf1b in U2-OS cells analyzed by immunofluorescence. Cyclophilin D was used as mitochondrial marker. Scale bar, $10 \mu \mathrm{m}$.

Carbonate extraction of isolated human mitochondria showed that RCF1a and $b$ were recovered in the membrane fraction along with other membrane proteins, TOM70 and TIM23, while the peripheral membrane protein TACO1 was recovered in the soluble fraction (Figure 3.11A). This is consistent with the prediction that RCF1a and RCF1b have two transmembrane domains. When isolated mitochondria were treated with protease, both proteins remained resistant (Figure 3.11B). Upon disruption of the outer membrane by osmotic swelling, RCF1b became accessible to protease, while RCF1a remained protected and no stable degradation products were detected (Figure 3.11C). These data demonstrate that RCF1a and b are integral inner membrane proteins of mitochondria of which RCF1b is exposed to the intermembrane space, thus displaying similar topology as yeast Rcf1. 
A

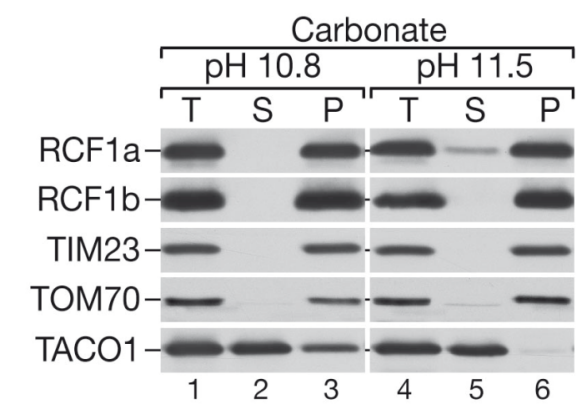

B

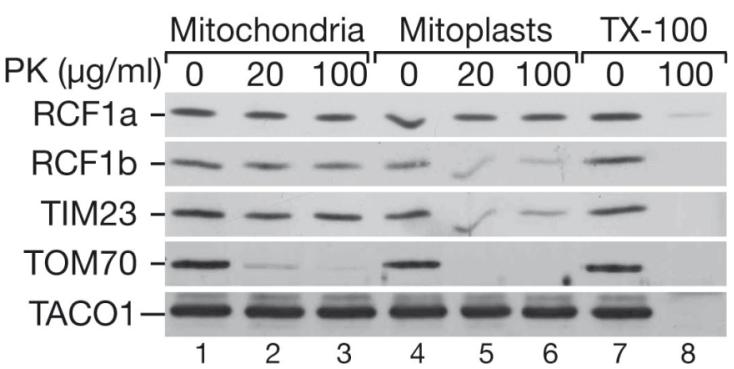

C

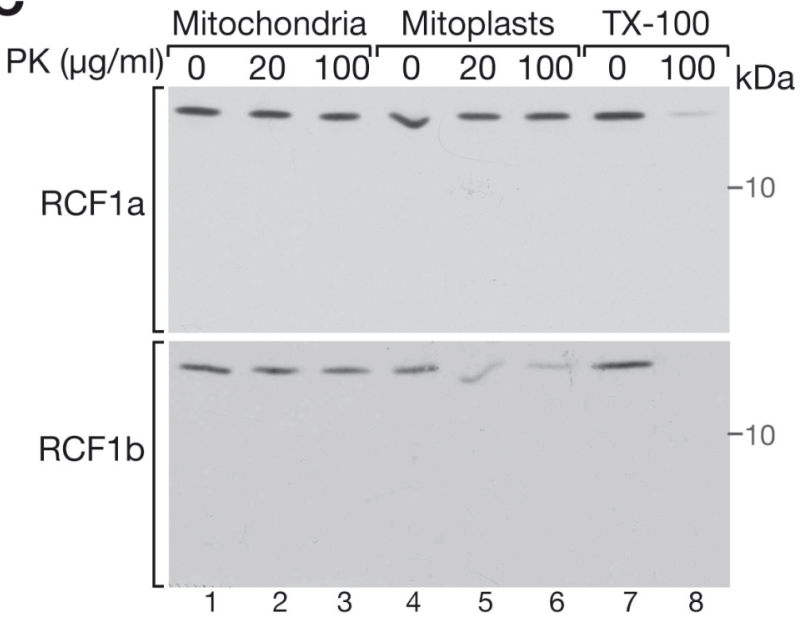

Figure 3. 11 RCF1a and RCF1b, are localised to the inner membrane of mitochondria. (A) Mitochondria isolated from HEK-293 cells were subjected to carbonate extraction at $\mathrm{pH} 10.8$ or 11.5 , followed by separation into pellet $(\mathrm{P})$ and supernatant $(\mathrm{S})$ by ultracentrifugation at $100000 \mathrm{~g}$; total $(\mathrm{T})$. Samples were analyzed by SDS-PAGE and immunodecoration. (B) Isolated mitochondria were left untreated, turned to mitoplasts by hypoosmotic swelling (mitoplasts) or lysed with Triton X-100, and treated with different concentrations of proteinase $\mathrm{K}$, followed by SDS-PAGE analyses and Western-blotting. (C) Blot portions below RCF1a and RCF1b are presented. No proteolytic fragments of RCF1a and RCF1b were detected.

\subsubsection{Human RCF1a and RCF1b proteins are complex IV components}

To investigate the association of RCF1a and $b$ with complex IV in human mitochondria, two different approaches were employed. First, isolated human mitochondria were solubilized and analyzed by BN-PAGE. Western-blotting revealed that most of RCF1a containing complexes co-migrated with monomeric complex IV and a portion of RCF1a was also present in respiratory chain supercomplexes (Figure 3.12A). The antibody against RCF1b was unable to detect the protein in BNPAGE analyses. Hence, radiolabeled RCF1b was imported into isolated human mitochondria. The protein assembled into a complex that co-migrated with the complex IV monomer. These results designated RCF1a and $b$ to interact with complex IV, and to further support these findings, human mitochondria were 
solubilized with digitonin and subjected to immunoprecipitation with antibodies against holo-complex IV and the $\mathrm{F}_{1} \mathrm{~F}_{0}-\mathrm{ATPase}$ as a control. Anti-complex IV antibodies precipitated $\mathrm{COX} 1$ and $\mathrm{COX} 6 \mathrm{~A} 1$ while anti- $\mathrm{F}_{1} \mathrm{~F}_{\mathrm{o}}$-ATPase antibodies precipitated $F 1 \beta$, indicating efficient isolation of the corresponding complexes. RCF1 $a$ and $b$ were specifically recovered in the anti-complex IV precipitate (Figure 3.12B). Taken together, RCF1a and RCF1b are constituents of complex IV in human mitochondria.
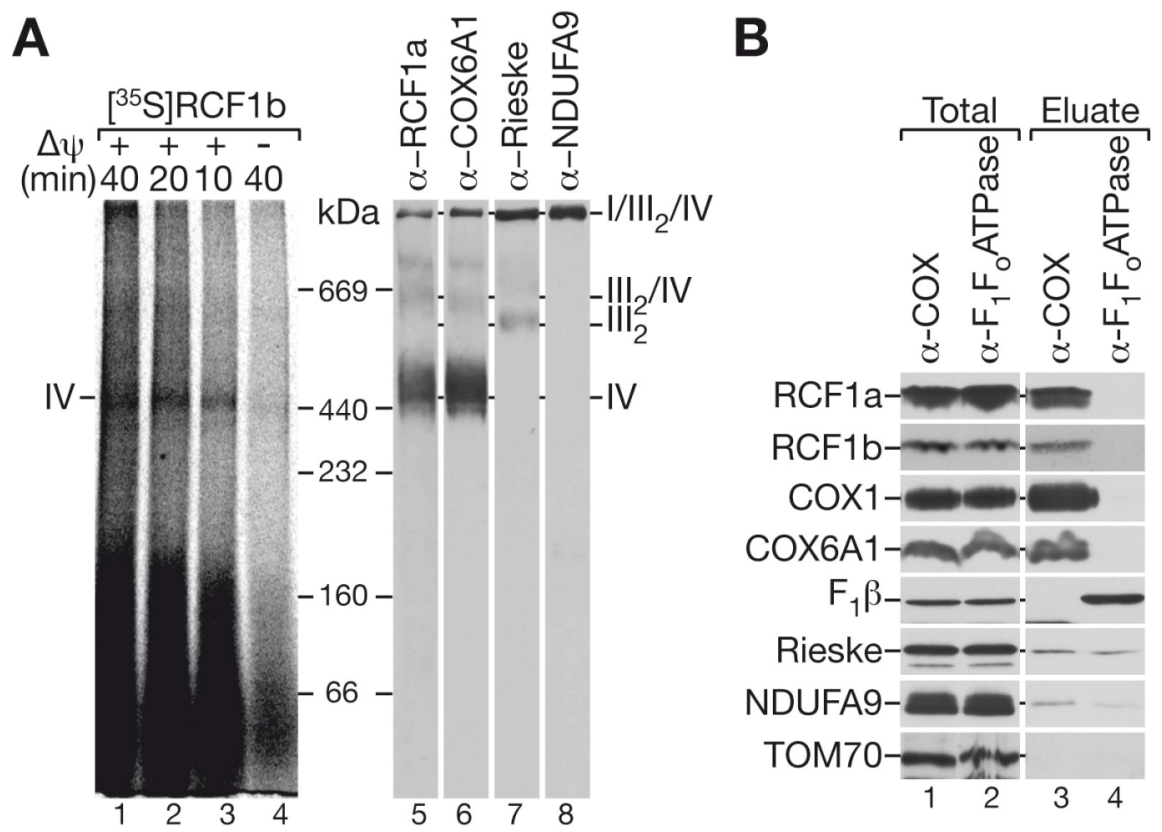

Figure 3. 12 RCF1a and RCF1b interact with cytochrome oxidase. (A) Radiolabelled RCF1b was imported into mitochondria isolated from HEK-293 cells. Upon treatment with proteinase $\mathrm{K}$ and digitonin-solubilization, samples were analyzed by BN-PAGE and digital autoradiography. For comparison, HEK-293 mitochondria were solubilized in digitonin buffer, analyzed by BN-PAGE and immunodecoration. (B) Coimmunoprecipitation of cytochrome oxidase (COX) and $F_{1} F_{\circ}$ ATPase from mitochondria isolated from HEK-293 cells after digitonin-solubilization. Samples were analyzed by SDS-PAGE and Westernblotting. Total, $1.5 \%$; eluate, $100 \%$. 


\section{2. ref1 $\Delta$ cells are growth deficient when grown on non- fermentable carbon sources}

To analyze the roles of Rcf1 and Rcf2 in regards to respiratory chain function, RCF1and RCF2-deletion mutants were generated and analyzed for growth on fermentable and non-fermentable media. $r c f 1 \Delta$ exhibited a growth defect on non-fermentable carbon sources and when plates were grown under low oxygen conditions the phenotype was enhanced, indicating their sensitivity to hypoxia (Figure 3.13A). The phenotype was compared to that of the respiratory deficient mutant missing mature complex IV because of the absence of the core subunit, Cox4, and to cox13 13 cells, affected in a subunit localized at the periphery of complex IV (Tsukihara et al., 1996), of which the growth behavior has been shown to be similar to wild type (Taanman and Capaldi, 1993). The growth defect on non-fermentable medium and normoxia of rcf1 $\Delta$ was less striking than that of $\operatorname{cox} 4 \Delta$, but more pronounced as compared to growth of cox $13 \Delta$ cells. In contrast to $r c f 1 \Delta$ cells, the growth behavior of $r c f 2 \Delta$ mutant cells was similar to that of $\operatorname{cox} 13 \Delta$ (Figure 3.13A). Additional evidence for $r c f 1 \Delta$ sensitivity to hypoxia came from growth analyses of wild type and $r c f 1 \Delta$ cells grown in a batch fermentor. Cells were cultivated on fermentable medium and their growth behavior was followed over time under hypoxic and normoxic conditions. Under normoxic growth conditions, the division time of both strains was similar, but when grown under hypoxia $r c f 1 \Delta$ cells displayed a significantly longer division time, as compared to wild type (Figure 3.13B). As cells lacking Rcf1 were able to grow on non-fermentable carbon sources, albeit with reduced efficiency in comparison to wild type cells, it is likely that these cells possess respiratory chain, but that it is functionally compromised. Because Rcf1 is complex IV component it was suggested that it is the complex which function is affected in $r c f 1 \Delta$ cells. 

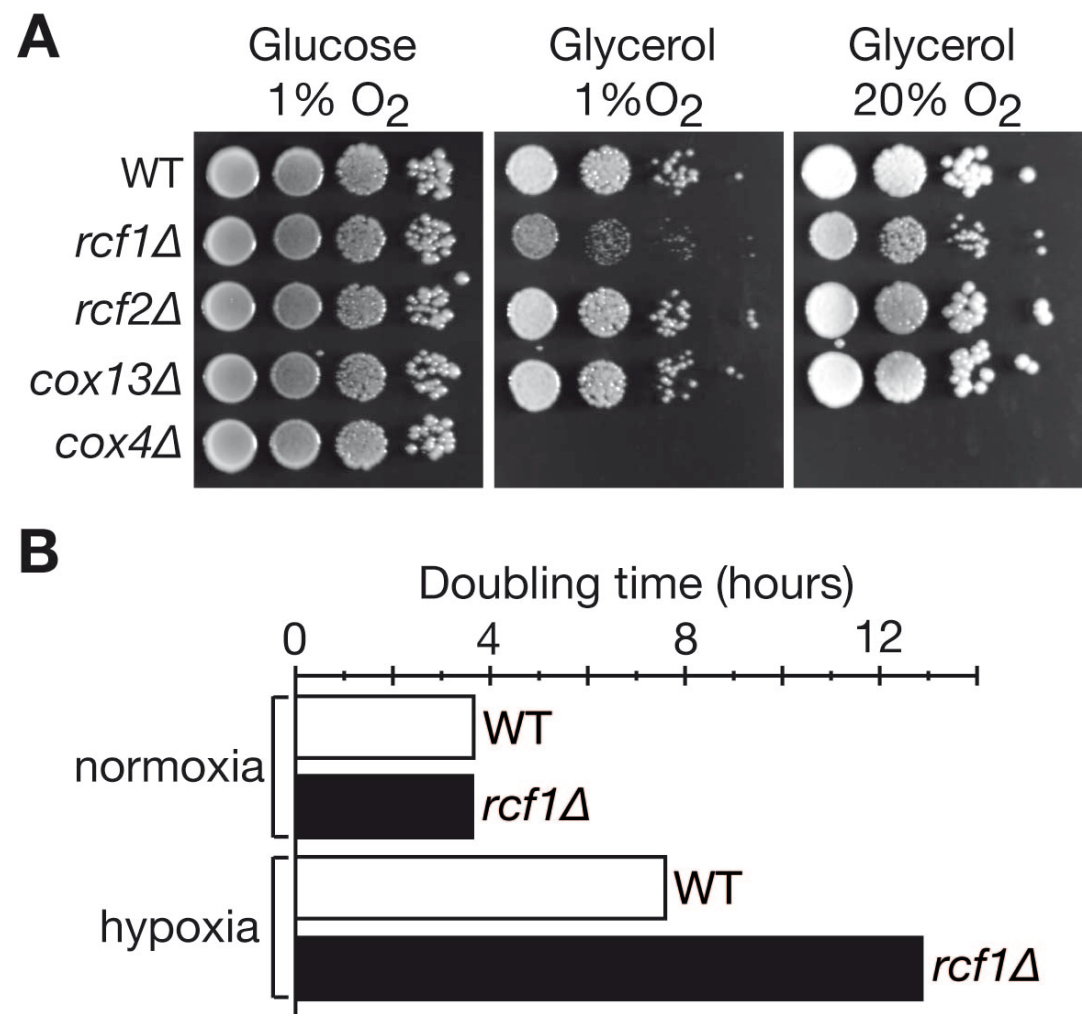

Figure 3. 13 Growth analyses of $r c f 1 \Delta$ and $r c f 2 \Delta$ cells. (A) WT, $r c f 1 \Delta, r c f 2 \Delta, \operatorname{cox} 13 \Delta$ and $\operatorname{cox} 4 \Delta$ cells were subjected to serial dilutions and spotted onto fermentable (glucose) and non-fermentable (glycerol) media. Plates were incubated at $30^{\circ} \mathrm{C}$ under $1 \%$ or $20 \%$ oxygen concentration. (B) Growth rate of WT and $r c f 1 \Delta$ cells was analyzed in fermentor system. Cells were grown either under normoxic or hypoxic conditions.

\subsection{Cox13 steady state protein levels are reduced in rcf1 $\Delta$}

If the structure of complex IV in $r c f 1 \Delta$ cells is not impaired, then the amounts of the constituents of this enzyme should be unaffected. To determine whether this was the case, steady-state protein levels analyzed by SDS-PAGE and Western-blotting were compared between wild type, $\operatorname{rcf} 1 \Delta$ and $\operatorname{rcf} 2 \Delta$ mitochondria. Most of the proteins, such as the outer mitochondrial membrane proteins (Tom70 and Tom40), matrix protein Aco1, complex III components (Cyt1, Rip1, Qcr8) and complex IV (Cox1, Cox2, Cox3, Cox4, Cox6), were similar in all three strains. However, $\operatorname{rcf} 1 \Delta$, and not rcf2 $\Delta$ mitochondria displayed a significantly reduced amount of Cox13 (Figure 
3.14A). When steady-state levels of various mitochondrial proteins were assayed in cox13 $\Delta$ cells, no significant difference, as compared to wild type, was observed and Rcf1 levels were not elevated (Figure 3.14B). These results suggested that Rcf1 influences either stability or expression of Cox13.
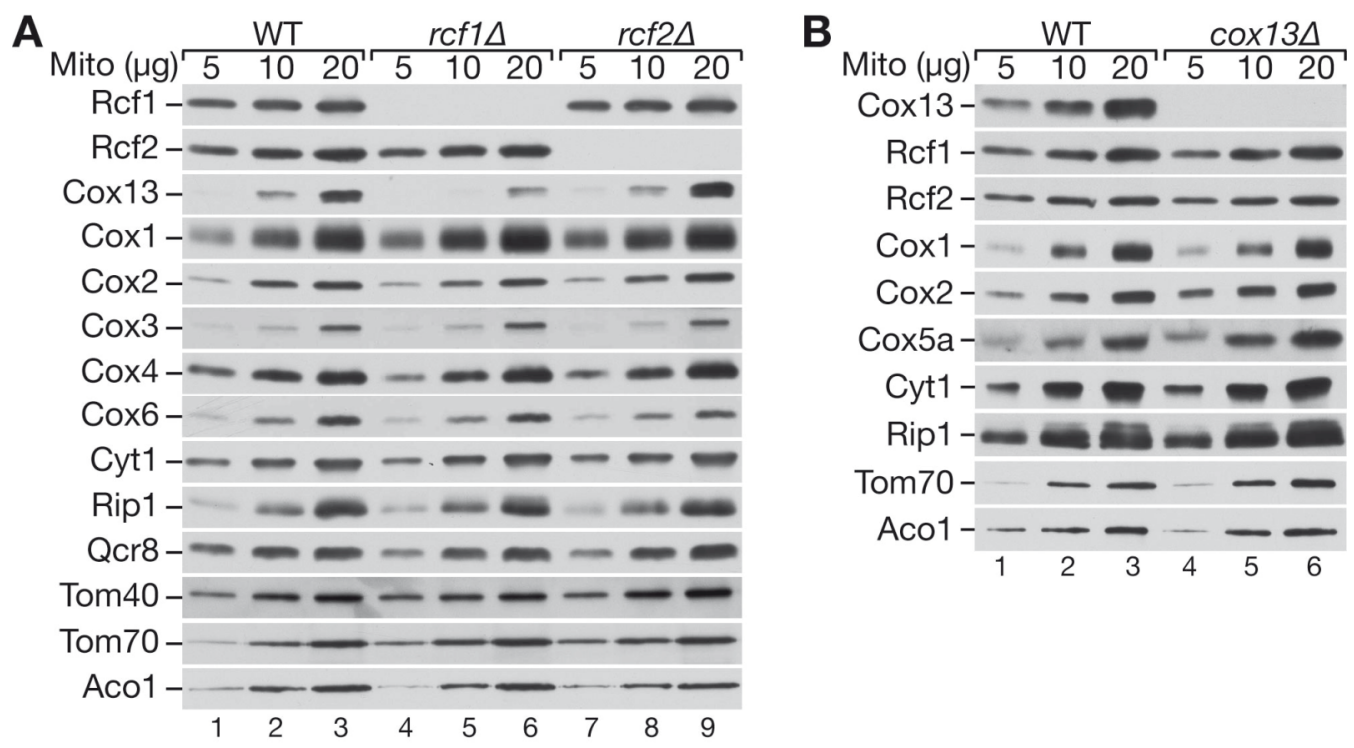

Figure 3. 14 Steady state protein levels analysis of WT, $\operatorname{rcf} 1 \Delta, \operatorname{rcf} 2 \Delta$ and $\operatorname{cox} 13 \Delta$ mitochondria. Mitochondria isolated from WT, $r c f 1 \Delta$ and $r c f 2 \Delta$ strains (A) WT and $\operatorname{cox} 13 \Delta$ (B) strains were analyzed by SDS-PAGE and immunodecoration against indicated proteins.

\subsection{Loss of Rcf1/Cox13 affects overall oxygen consumption in mitochondria}

\subsubsection{Establishment of oxygen consumption assays}

As no major differences in protein content of the respiratory chain in $r c f 1 \Delta$ cells were observed, another possible explanation for the observed growth defect was that the functionality of the respiratory chain was compromised. To assess this, an assay measuring oxygen consumption in isolated mitochondria was established. To optimize conditions, initial measurements were performed with mitochondria isolated from a wild type strain. After the polarograph had been calibrated, respiratory buffer 
(containing excess phosphate) was introduced into the chamber followed by the addition of isolated mitochondria. Subsequently, substrates such as NADH and ADP (causes rapid increase in oxygen uptake as the ADP is converted to ATP) were added. Under conditions where the substrate is present, but ADP is limiting, mitochondria exist in "state 4" respiration, while respiration in the presence of excess ADP is referred as "state 3". After the ADP is exhausted, mitochondria return to "state 4" (Figure 3.15A). Respiratory efficiency between different strains was determined by comparing the oxygen consumption rates, following "state 3 ".

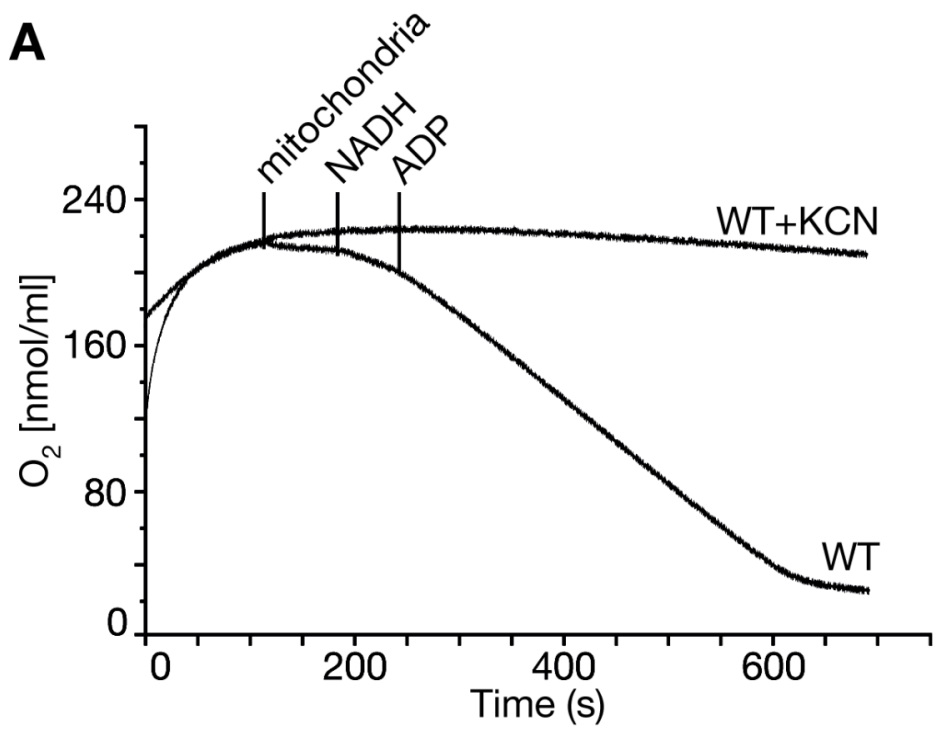

B

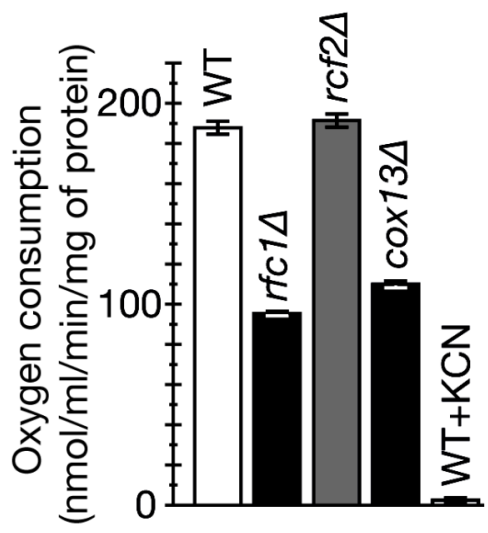

Figure 3. 15 Oxygen consumption in rcf1 $\Delta$ mitochondria is reduced. (A) Oxygen consumption recording charts using intact mitochondria isolated from WT strains or mitochondria treated with $10 \mathrm{mM} \mathrm{KCN}$. As substrate NADH was used. (B) Oxygen consumption rates in mitochondria isolated from indicated strains were measured as explained in section 2.6.9. Means of three independent measurements (SEM, $n=3$ ).

\subsection{2. $\quad r c f 1 \Delta$ and $\operatorname{cox} 13 \Delta$ mitochondria displayed reduced oxygen consumption rates}

The established assay was employed to test the $\mathrm{O}_{2}$ consumption in wild type and mutant mitochondria. The oxygen consumption rate of mitochondria isolated from the $r c f 1 \Delta$ strain was significantly reduced in comparison to wild type mitochondria, but 
similar to that of mitochondria lacking Cox13 (Figure 3.15B). The overall oxygen consumption rate of $r c f 2 \Delta$ mitochondria was in the range of oxygen uptake by wild type mitochondria (Figure 3.15B). These results indicate respiratory chain dysfunction in $r f f 1 \Delta$ and $\operatorname{cox} 13 \Delta$ mitochondria.

\subsection{In the absence of Rcf1/Cox13, cytochrome oxidase activity is altered}

\subsubsection{Establishment of an enzyme activity assays}

The approach to assess the specific activity of individual respiratory chain complexes was established in Mick et. al. 2010. In the course of these experiments wild type, coa3 $\Delta$ and cox $14 \Delta$ mitochondria were used. Coa3 and Cox14 are assembly factors of complex IV that are responsible for formation of the first cytochrome oxidase assembly intermediate and are involved in translational regulation of Cox1 (Barrientos et al., 2004; Mick et al., 2010). This method enables the individual spectrophotometrical monitoring of complex III and IV activity, based on the rate of cytochrome $c$ reduction/oxidation. The $b c_{1}$ complex activity of both mutants, as compared to wild type, was similar, while the cytochrome oxidase activity was dramatically reduced in coa3 $\Delta$ and cox $14 \Delta$ mitochondria (Figure 3.16). The observed results were consistent with an early cytochrome oxidase assembly role of Coa3 and Cox14, further supporting this notion, their absence blocks complex formation. As these complexes control the respiratory rate, an internal control assay allowing for the examination of an enzyme/complex independent of the respiratory chain was also established. The enzyme of choice, malate-dehydrogenase, which converts oxaloacetate to malate can be followed spectrophotometrically. As expected, the enzyme activities of the tested strains were comparable (Figure 3.16). 

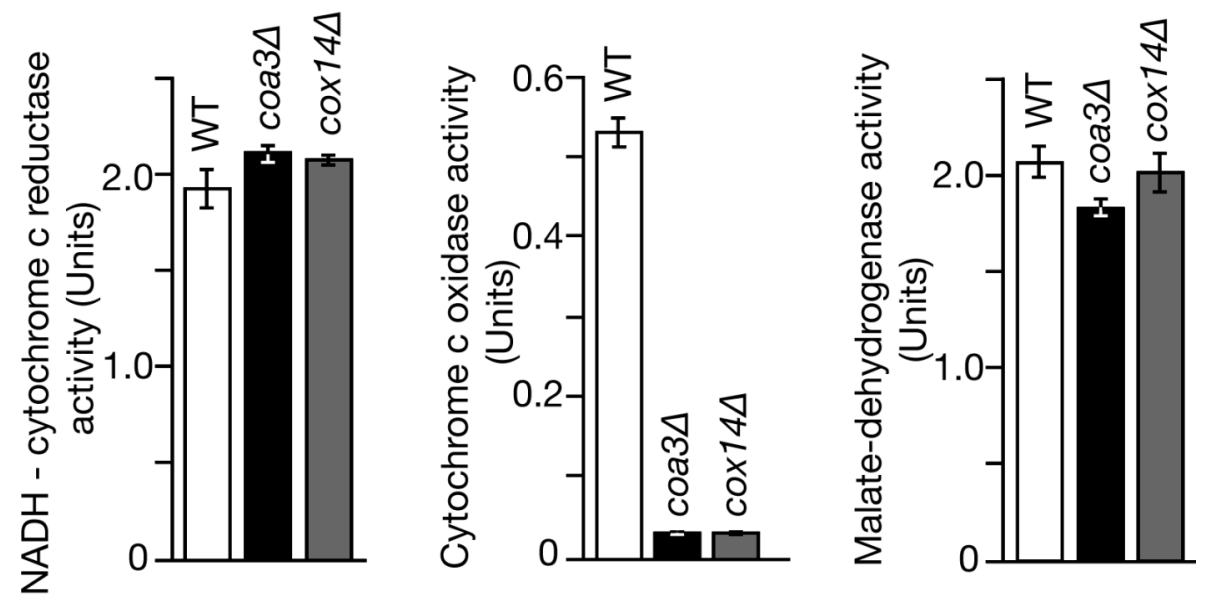

Figure 3. 16 Enzyme activities of mitochondria isolated from WT, coa3 $\Delta$ and $\operatorname{cox} 14 \Delta$ cells. Enzyme assays of NADH-cytochrome $c$ reductase, cytochrome oxidase and malate dehydrogenase were performed with WT, coa3 $\Delta$ and cox14 $\Delta$ isolated mitochondria as explained in section 2.6.8. Means of three independent measurements (SEM, $n=3$ ).

As an additional control, an assay examining total aconitase activity in isolated mitochondria was also established. The method is based on the enzyme's ability to catalyze the formation of isocitrate from cis-aconitate. This reaction can be monitored spectrophotometrically as a decrease in absorbance.

\subsection{2. $\quad r c f 1 \Delta$ displays reduced cytochrome oxidase activity while both mutants, $r c f 1 \Delta$ and $r c f 2 \Delta$, have reduced aconitase activity}

In order to evaluate single enzyme activities in isolated mitochondria from wild type, $r c f 1 \Delta, r c f 2 \Delta$ and $\operatorname{cox} 13 \Delta$ cells, the established enzyme activity assays were utilized. The malate-dehydrogenase and the $b c_{1}$ complex activities were similar in all tested strains. When these strains were analyzed for cytochrome oxidase activity, $r c f 1 \Delta$ and $\operatorname{cox} 13 \Delta$ mitochondria displayed a significant reduction, while $\operatorname{rcf} 2 \Delta$ mitochondria were not affected as compared to wild type (Figure 3.17A). These results indicate that Rcf1 and Cox13 are necessary for cytochrome oxidase activity and their deletion leads to a common phenotype, while Rcf2 appears not to be essential for cytochrome oxidase activity under the conditions used here. 
A

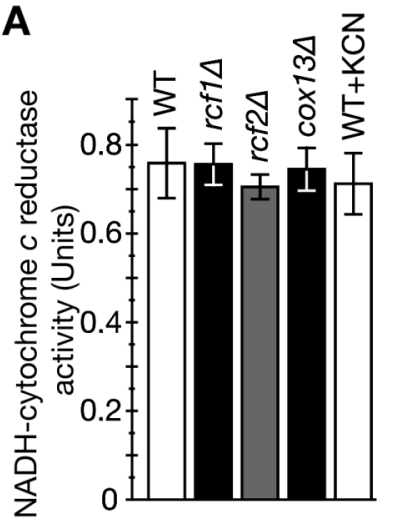

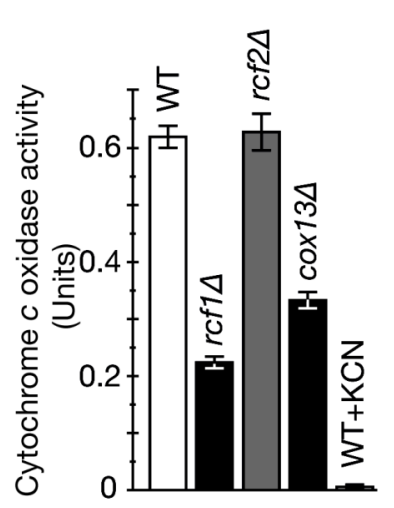

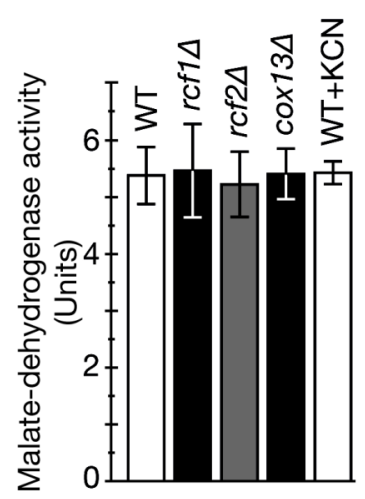

B

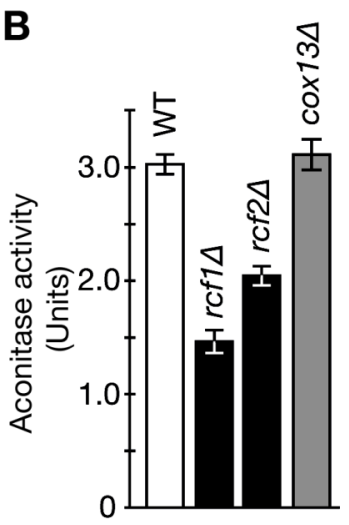

Figure 3. 17 Enzyme activities of WT, $r c f 1 \Delta, r c f 2 \Delta$ and $\operatorname{cox} 13 \Delta$ mitochondria. (A) Mitochondrial enzyme activities of NADH-cytochrome $\mathrm{c}$ reductase, cytochrome $\mathrm{c}$ oxidase and malate dehydrogenase assessed in indicated strains. (B) Aconitase activities of mitochondria isolated from indicated strains. (A), (B) Means of three independent measurements (SEM, $n=3$ ).

When aconitase activity was assayed, a significant reduction was detected in $r c f 1 \Delta$ and $r c f 2 \Delta$ mitochondria (Figure 3.17B) although the relative amount of aconitase (Aco1) in both strains was similar in comparison to wild type (Figure 3.14A). The active site of aconitase contains a $4 \mathrm{Fe}-4 \mathrm{~S}$-cluster, highly sensitive to superoxide $\left(\mathrm{O}_{2}{ }^{-}\right)$(Raha and Robinson, 2000) and a decrease/loss in its activity is considered a marker of increased reactive oxygen species (ROS) production. Hence, the observed results suggested that $r c f 1 \Delta$ and $r c f 2 \Delta$ mitochondria have a defect and displayed increased ROS production.

\subsection{Mitochondria isolated from $\operatorname{rcf} 1 \Delta$ and $\operatorname{rcf} 2 \Delta$ cells display increased ROS production}

The overall ROS production in mitochondria can be followed fluorometrically over time by conversion of $\mathrm{H}_{2} \mathrm{DCFDA}$ (dichlorodihydrofluorescein diacetate) into highly fluorescent 2'7'-dichlorofluorescein. $\mathrm{H}_{2} \mathrm{DCFDA}$ is one of the most versatile compounds to detect a broad range of reactive oxygen species. When ROS production was assessed in wild type, $r c f 1 \Delta, r c f 2 \Delta$ and $\operatorname{cox} 13 \Delta$ mitochondria, all three mutants produced ROS to higher extent than wild type mitochondria. The levels of ROS generated in $r c f 1 \Delta$ and $r c f 2 \Delta$ mitochondria were severally increased 
compared to wild type mitochondria, whereas $\operatorname{cox} 13 \Delta$ mitochondria exhibited an intermediate phenotype (Figure 3.18B). In contrast, when ROS generation of mitochondria lacking the heme-containing core subunit Cox1 was analyzed, it resembled the wild type phenotype (Figure 3.18A). Therefore, Rcf1, Rcf2 and Cox13 can be characterized as complex IV proteins that protect mitochondria against increased ROS generation.
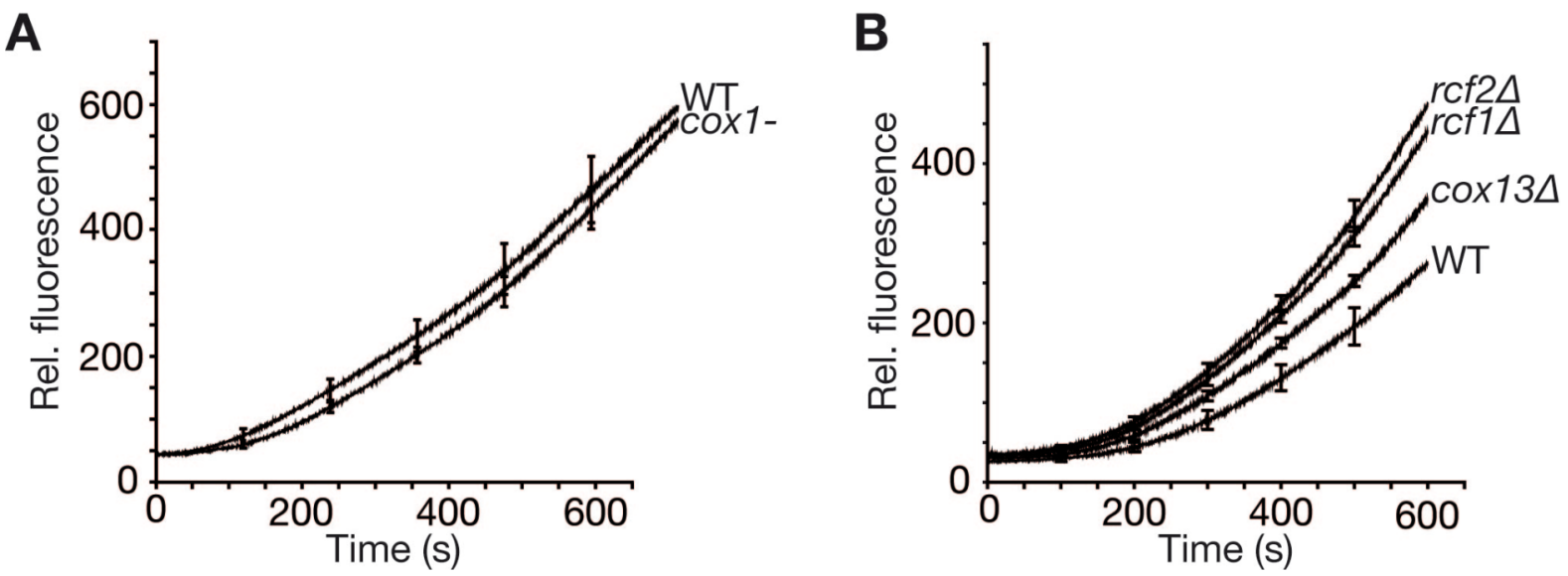

Figure 3. 18 rcf1 $\Delta$ and $r c f 2 \Delta$ mitochondria exert higher ROS production. (A), (B) Mitochondrial ROS production in indicated strains was measured as stated in section 2.6.10. Means of three independent measurements (SEM, $n=3$ ).

\subsection{The RCF1 deletion phenotype is partially rescued by human RCF1b}

Given the fact that yeast Rcf1 possess two human homologs, RCF1a and RCF1b, and that both of them associate with complex IV as in yeast, it was speculated that these two proteins could be functional homologues of the yeast protein. In order to assess this assumption, human genes RCF1a and RCF1b were tested for their ability to substitute for the lack of RCF1 in yeast. For these purpose plasmids carrying the human or yeast gene were transformed into an $r c f 1 \Delta$ strain and subsequently tested for their ability to complement its growth phenotype on nonfermentable medium. The growth defect of $r c f 1 \Delta$ was partially recovered by RCF1b 
expression, but not by RCF1a expression (Figure 3.19A). Yeast RCF1 could not completely restore the growth of $r c f 1 \Delta$, which can be explained by the fact that the plasmid expression of the gene was somewhat reduced, indicating that the amount of yeast Rcf1 was rate limiting. Thus, the competence of human and yeast RCF1 to complement increased production of ROS rcf1 $1 \Delta$ mitochondria was tested under nonlimiting expression levels. Expression of Rcf1 and RCF1b in rcf1 $1 \Delta$ cells reduced ROS production to a high extent in both cases, while RCF1a expression could not rescue the phenotype (Figure 3.19B). Therefore, RCF1b is a functional homolog of Rcf1 in yeast. 

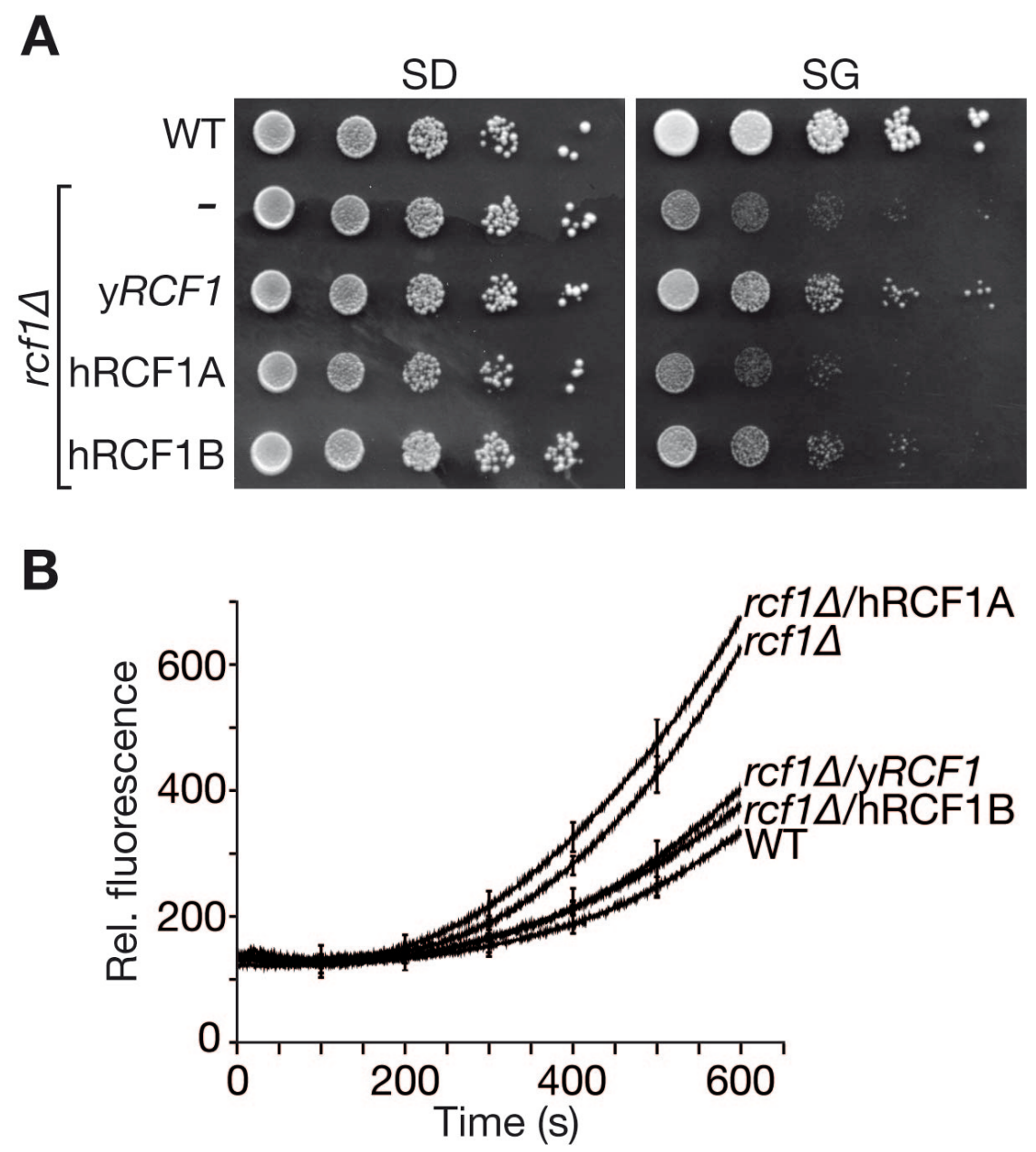

Figure 3. 19 hRCF1B can complement phenotype of $r c f 1 \Delta$. (A) WT, $r c f 1 \Delta$ and $r c f 1 \Delta$, complemented with yeast $\mathrm{YRCF} 1$ or human $\mathrm{hRCF} 1 \mathrm{~A}$ and $\mathrm{hRCF} 1 \mathrm{~B}$ genes, were diluted in ten-fold increments and spotted onto fermentable (SD, selective glucose) or nonfermentable (SG, selective glycerol) media. Plates were incubated at $30^{\circ} \mathrm{C}$. (B) ROS production assayed in mitochondria isolated from indicated strains. Means of three independent measurements (SEM, $n=3$ ).

\subsection{Assembly of Rcf2 and Cox13 is Rcf1 dependent}

The aforementioned results showed that steady-state protein levels of Cox13 were reduced in $r c f 1 \Delta$ mitochondria, which can be explained by the defect in import and assembly of the Cox13 subunit. To analyze this in more detail, Cox13, Rcf1, and Rcf2 were synthesized and $\left[{ }^{35} \mathrm{~S}\right.$-labeled in rabbit reticulocyte lysate, and in vitro imported/assembled into mitochondria isolated from wild type, $\operatorname{cox} 13 \Delta, \operatorname{rcf} 1 \Delta$, and 
rcf2 $\Delta$. Upon import, mitochondria were digitonin-solubilized and analyzed by $\mathrm{BN}-$ PAGE. Consistent with previous observations, Rcf2 assembled preferentially into supercomplexes in wild type mitochondria, while in mitochondria lacking Cox13 (Figure 3.20A) or Rcf1 (Figure 3.20B) its assembly was completely blocked. Observed defects in assembly could be explained as an indirect consequence of blocked precursor import. Therefore, the efficiency of precursor import into isolated mitochondria was additionally analyzed by SDS-PAGE, and it was found that Rcf2 imported similarly in mutant and wild type mitochondria (Figure 3.20A, B).

When Cox13 assembly was assayed in mitochondria isolated from wild type cells, efficient assembly into supercomplexes in a membrane-potential manner was observed (Figure 3.20C). Interestingly, assembly of Cox13 was not affected in an rcf $2 \Delta$ background and resembled wild type assembly (Figure 3.20C). Conversely, Cox13 assembly in $r c f 1 \Delta$ mitochondria was completely blocked (Figure 3.20D). As for Rcf2, import efficiency of Cox13 in wild type and mutant mitochondria was tested by SDS-PAGE, and shown not to be affected (Figure 3.20C, D). Lastly, in vitro import and assembly of Rcf1 in wild type, cox13 $\Delta$ and $r c f 2 \Delta$ mitochondria were analyzed. Compared to wild type mitochondria, import/assembly of the Rcf1 precursor was unaffected in both mutants (Figure 3.20E, F).

In summary, Rcf2's incorporation into supercomplexes is dependent on the presence of Rcf1 and Cox13 in these complexes. Moreover, efficient assembly of Cox13 relies on Rcf1, while Rcf1's integration into supercomplexes does not require Cox13 or Rcf2. Therefore, it appears that these three proteins assemble sequentially with Rcf1 as the first, followed by Cox13 and then Rcf2.

Figure 3. 20 Assemblies of Cox13 and Rcf2 into cytochrome c oxidase are Rcf1dependent. Radiolabeled Rcf2, Cox13 and Rcf1 were imported into isolated yeast mitochondria in the presence or absence of $\Delta \psi$ for indicated times and treated with proteinase K. Samples were lysed in $1 \%$ digitonin, analyzed by BN-PAGE or SDS-PAGE and digital autoradiography. Rcf2 were imported into WT/cox13 $\Delta$ mitochondria (A) and WT/rcf1 $\Delta$ mitochondria (B). Cox13 were imported into WT/rcf2 $\Delta$ mitochondria (C) and WT/rcf1 $\Delta$ mitochondria (D). Rcf1 were imported into WT/cox13 $\Delta$ mitochondria (E) and WT/rcf2 $\Delta$ mitochondria (F). 
A

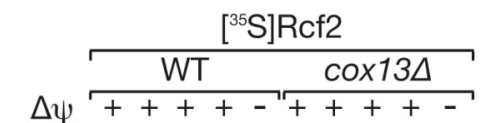

(min) 12510101251010

$\mathrm{III}_{2} \mathrm{IV}_{2-}$

III ${ }_{2} \mathrm{IV}$ -

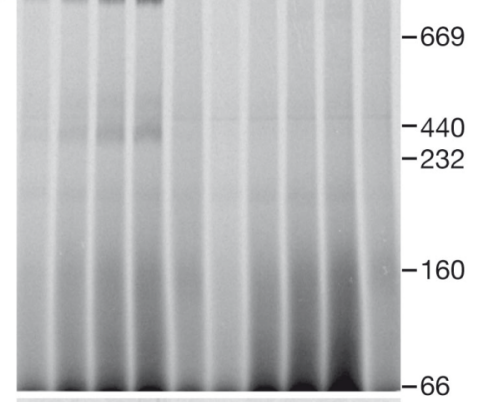

$\left[{ }^{35} \mathrm{~S}\right] \mathrm{Rcf} 2$

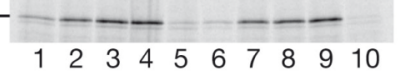

C $[35 \mathrm{~S}] \mathrm{Cox} 13$

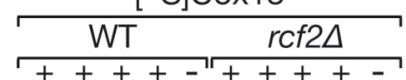

$\Delta \psi++++-++++-$ (min) 10521101052110

$\mathrm{III}_{2} \mathrm{IV}_{2-}$ $\mathrm{III}_{2} \mathrm{IV}-$

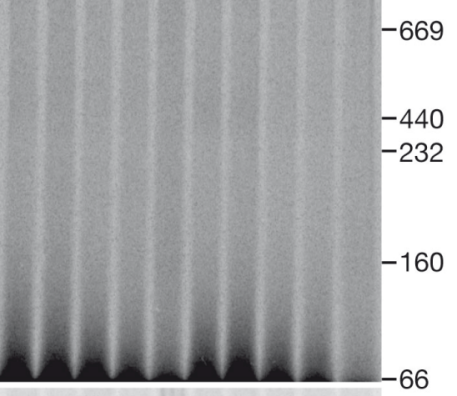

$\left.{ }^{35} \mathrm{~S}\right] \mathrm{Cox} 13-\equiv==-\frac{\mathrm{p}}{\mathrm{m}}$

E $\left[{ }^{35} \mathrm{~S}\right] \mathrm{Rcf} 1$

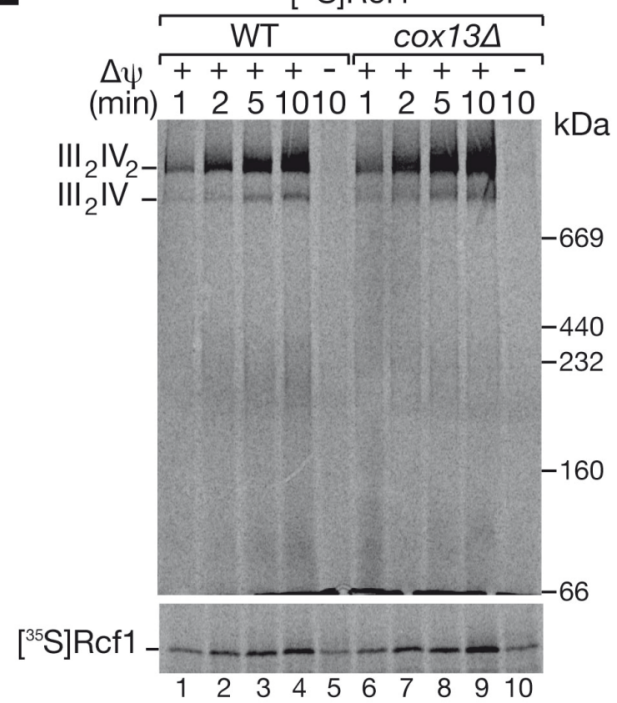

B

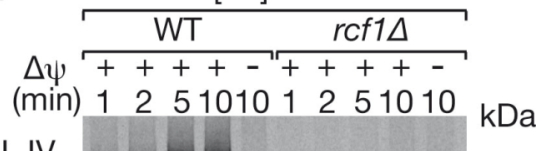

$\mathrm{III}_{2} \mathrm{IV}_{2}$

$\mathrm{III}_{2} \mathrm{IV}-$

669

440

$-232$

160

D

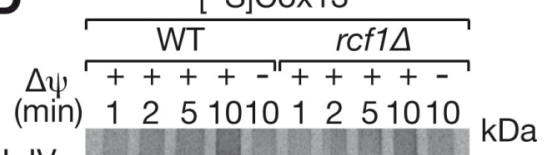

$\mathrm{III}_{2} \mathrm{IV}_{2-}$

$\mathrm{III}_{2} \mathrm{IV}-$

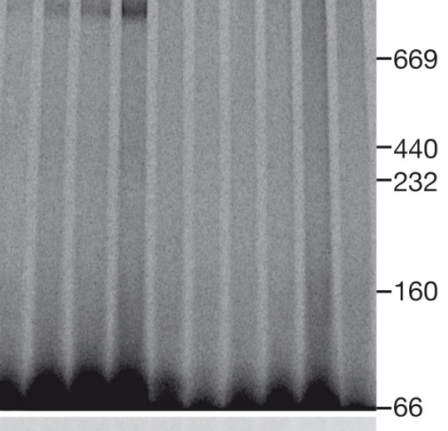

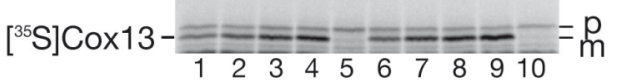

$\mathbf{F}$ $\left[{ }^{35} \mathrm{~S}\right] \mathrm{Rcf} 1$

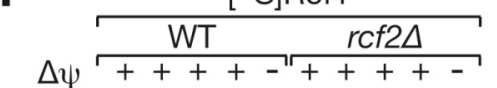

(min) $12510101251010 \mathrm{kDa}$

$\mathrm{III}_{2} \mathrm{IV}_{2-}-\mathrm{N}_{-\mathrm{m}}=$

$\mathrm{III}_{2} \mathrm{IV}$

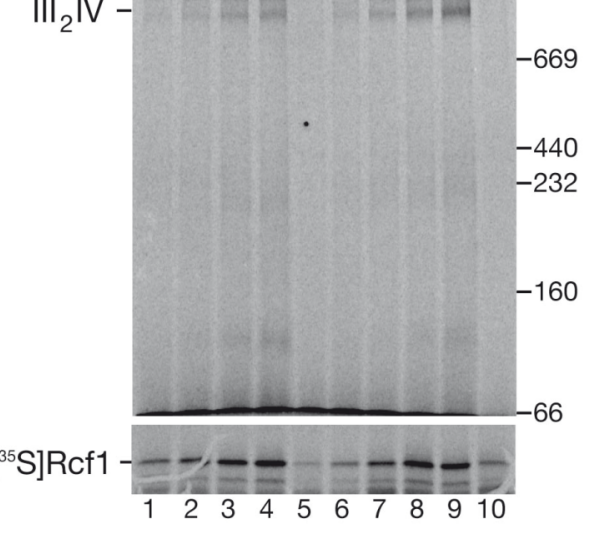




\subsection{Formation of the supercomplexes in mitochondria requires Rcf1}

As evidenced above, steady-state levels of complex III and IV constituents were not changed in any of the three mutants, with the exception of Cox13 in $\operatorname{rcf} 1 \Delta$. To analyze whether the formation of respiratory chain complexes at steady state was affected in $r c f 1 \Delta, r c f 2 \Delta$ and $\operatorname{cox} 13 \Delta$, mitochondria isolated from indicated strains were solubilized in digitonin buffer and complexes were separated by BN-PAGE. Respiratory chain complexes III and IV were present in all mutant mitochondria and formation of supercomplexes was not affected in $r c f 2 \Delta$ and $\operatorname{cox} 13 \Delta$ (Figure 3.21A). In contrast, BN-PAGE analyses of $r c f 1 \Delta$ mitochondria revealed a decrease in the amount of $\mathrm{II}_{2} \mathrm{IV}$ complexes and an increase in complex $\mathrm{II}_{2} \mathrm{IV}$ and $\mathrm{II}_{2}$ levels, as compared to wild type mitochondria (Figure 3.21A). The observed defect resembled that of taz1 $1 \Delta$ mitochondria, affected in cardiolipin biosynthesis and therefore in supercomplex formation (Brandner et al., 2005) (Figure 3.21B). However, the defect was more dramatic in $r c f 1 \Delta$ mitochondria, suggesting that the absence of Rcf1 either leads to the destabilization and dissociation of $\mathrm{II}_{2} \mathrm{IV}_{2}$ complex or to inefficient formation. Expression of yeast RCF1 in rcf1 $1 \Delta$ cells could rescue the observed phenotype, but expression of human RCF1a and b could not (Figure 3.21C). Although the assembly of Cox13 and Rcf2 was completely impaired in the absence of Rcf1, at steady state minute amounts of both proteins were detected in respiratory chain complexes (Figure 3.21A, lanes 18 and 22), indicating that in vivo these two proteins can incorporate into supercomplexes to some extent. Thus, Rcf1 can be characterized as a protein necessary for efficient Cox13 and Rcf2 assembly but not essential. In addition, Rcf2 could not be detected in supercomplexes of cox13A mitochondria, but only in an unassembled form in the low molecular weight range of BN-PAGE (Figure 3.21A, lane 20). This finding further supports the hypothesis that the assembly of Rcf2 is dependent on the presence of Cox13. 


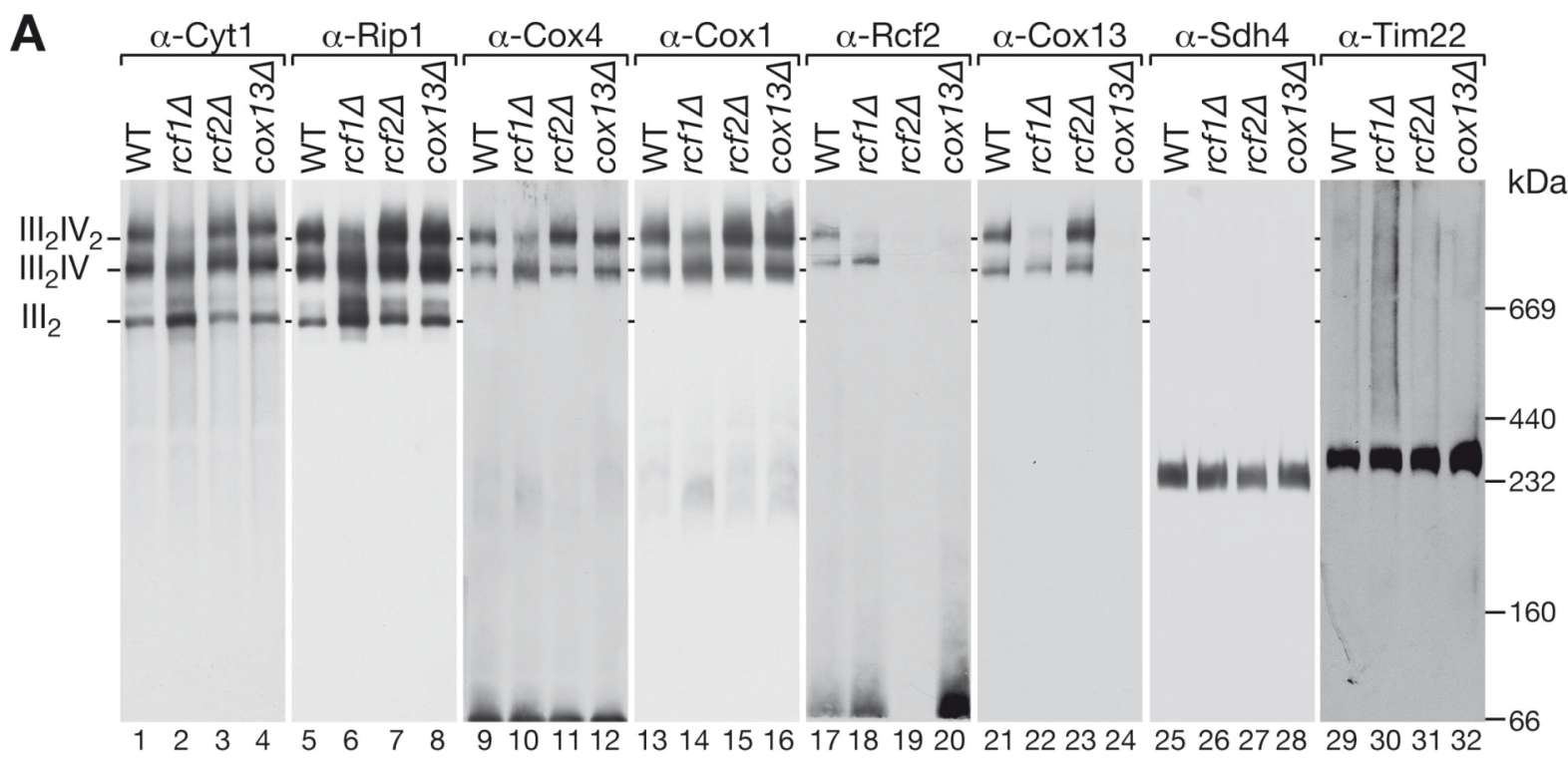

B

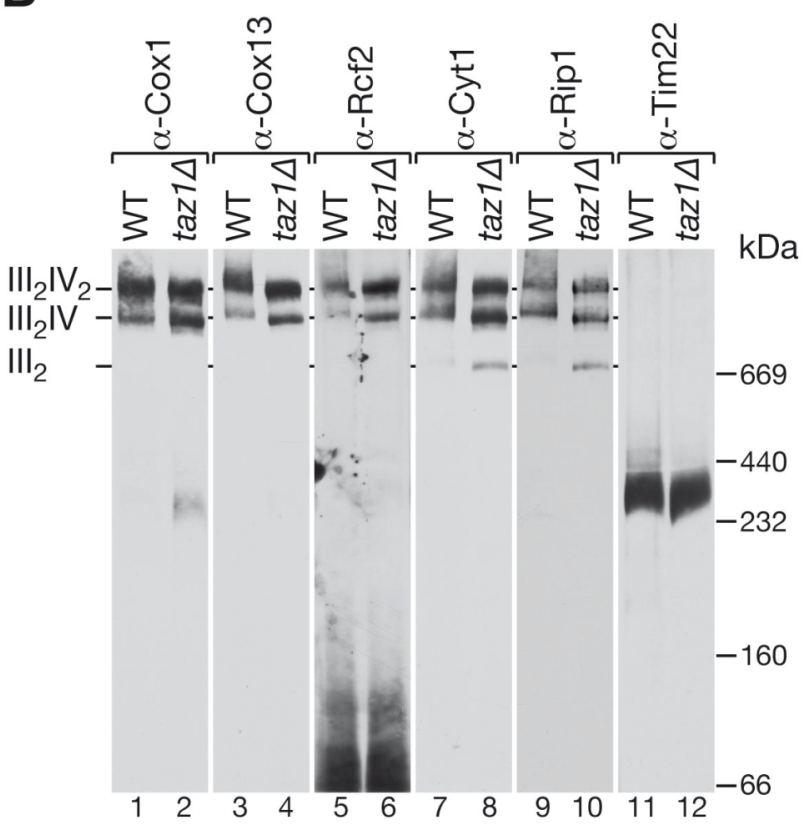

C

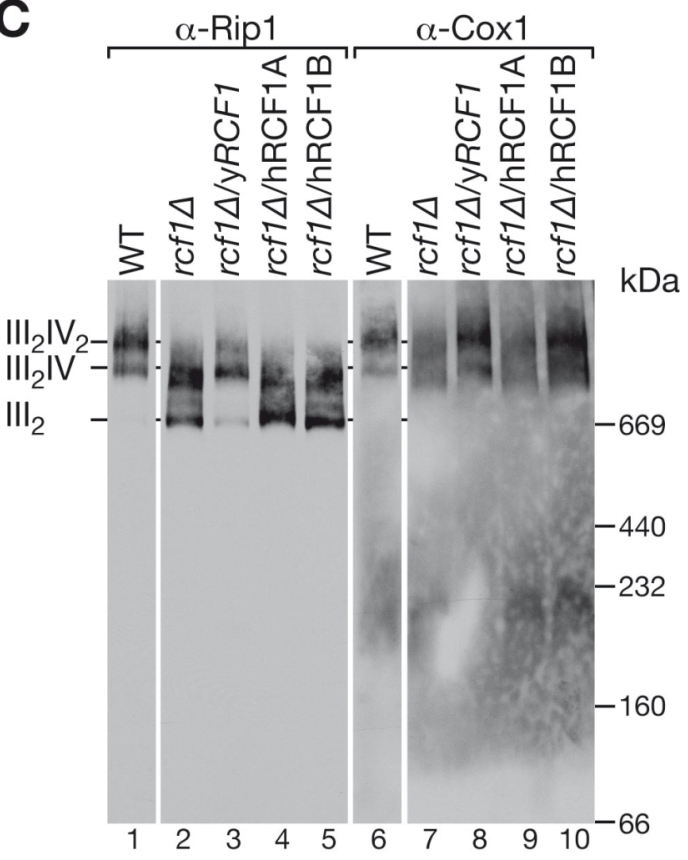

Figure 3. 21 Supercomplex formation depends on Rcf1. (A) Mitochondria isolated from indicated strains were lysed in $1 \%$ digitonin and analyzed by BN-PAGE and immunodecoration against indicated proteins. (B) WT and taz1s mitochondria were treated and analyzed as in (A). (C) Mitochondria isolated from indicated strains were analyzed as in (A).

Rcf1 promotes the association of complex IV to complex $\mathrm{III}_{2}$ and the formation of supercomplexes, but most likely is not the only factor involved in this process. If this were the case, in the absence of Rcf1 full dissociation of supercomplexes into 
monomeric complex IV and dimeric complex III should occur. Since the dissociation is only partial, it can be explained in two ways:

- mitochondria lacking Rcf1 have less complex IV which shifts the equilibrium between supercomplexes to the formation of $\mathrm{III}_{2} \mathrm{IV}$ complexes;

- mitochondria possess different populations of complex IV which differ in their composition and just a fraction of complex IV monomers containing Rcf1 are affected.

If the former scenario was correct, it would be expected that steady state levels of complex IV subunits would be reduced in rcf1 $1 \Delta$ mitochondria, however, no such differences between $r c f 1 \Delta$ and wild type protein levels were observed.

\subsection{Supercomplexes formed in mitochondria differ in regard to their composition}

If complexes IV present in mitochondria were not identical and if all could associate with complex $\mathrm{II}_{2}$, then the supercomplexes formed should also be heterogenic. To test this, yeast strains expressing functional C-terminal ZZ-tagged versions of Cox4, Cox13 and Rcf2 were generated and mitochondria isolated from these strains were solubilized in digitonin-containing buffer, followed by purification of $\operatorname{Cox} 4^{\mathrm{ZZ}}, \operatorname{Cox} 13^{\mathrm{ZZ}}$ and $\mathrm{Rcf} 2^{\mathrm{ZZ}}$ via IgG chromatography. Proteins co-isolated with $\operatorname{Cox} 4^{\mathrm{ZZ}}$, Cox13 ${ }^{\mathrm{ZZ}}$ and $\mathrm{Rcf}^{2 Z}$ were analyzed by SDS-PAGE and Western-blotting, revealing specific purification of respiratory chain supercomplexes (Figure 3.22A). Under the conditions used here, Cox4 ${ }^{\mathrm{ZZ}}$ and Cox $13^{\mathrm{ZZ}}$ efficiently isolated respiratory complex III and IV components, whereas Rcf2 ${ }^{\mathrm{ZZ}}$ led to the isolation of only a fraction of these proteins (Figure 3.22A). Also, when elution fractions of the same experiment were analyzed by BN-PAGE, it was noted that the amount of supercomplexes co-isolated with $\mathrm{Rcf}^{2 \mathrm{ZZ}}$ was less in comparison to that of $\mathrm{Cox} 4^{\mathrm{ZZ}}$ and $\mathrm{Cox} 13^{\mathrm{ZZ}}$ purifications (Figure 3.22B). Further more, this finding was in agreement with the BN-PAGE analyses in which Rcf2 could be detected in its monomeric, unassembled form at the low molecular range of the gel (Figure 3.21A). Taken together, Rcf2 can be characterized as a substoichiometric subunit of supercomplexes. 
A

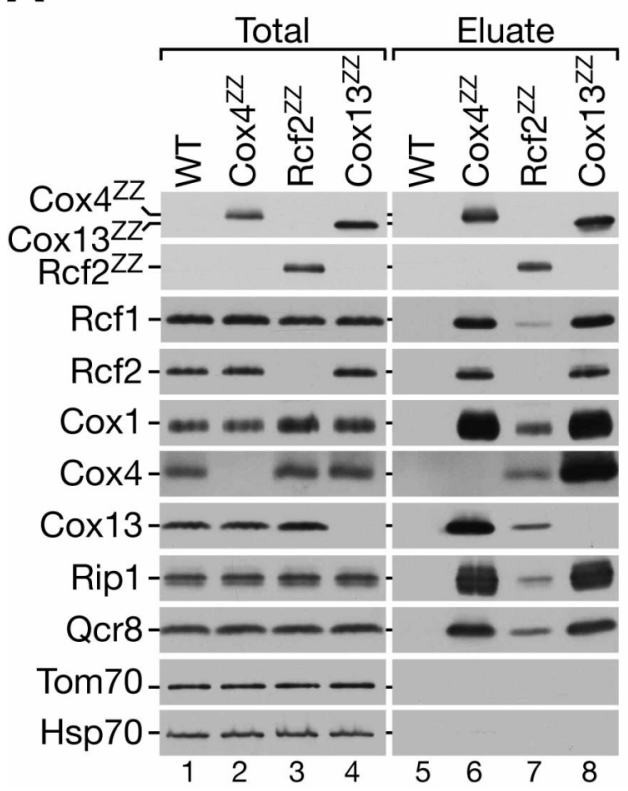

B

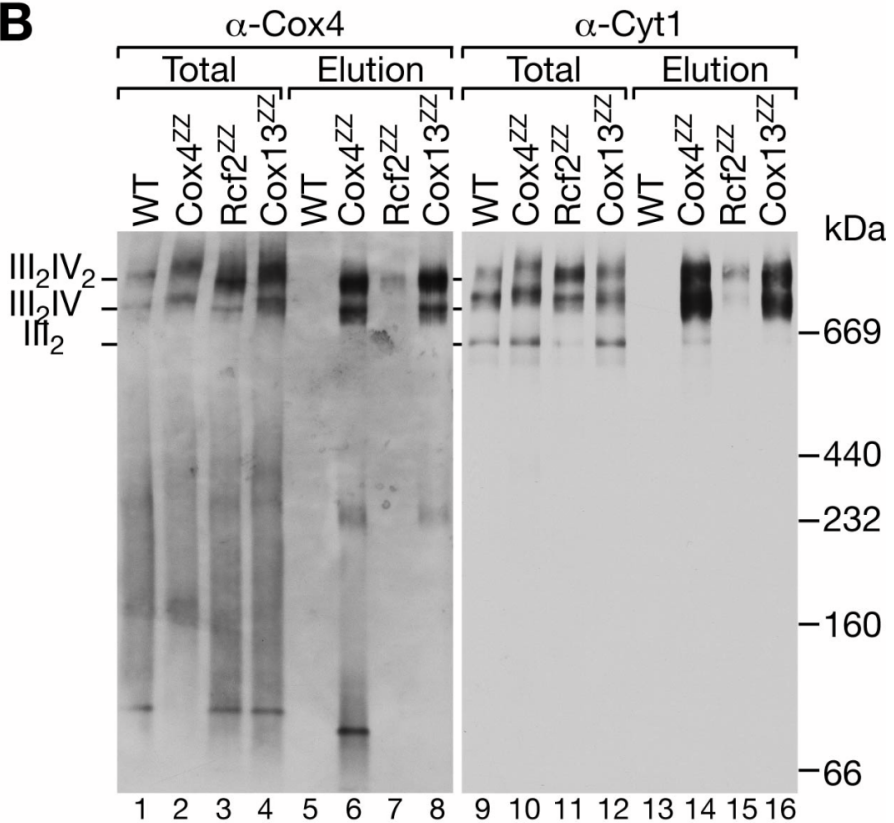

Figure 3. 22 Isolation of supercomplexes via Cox $4^{\mathrm{ZZ}}$, Cox $13^{\mathrm{ZZ}}$ and $\mathrm{Rcf} 2^{\mathrm{ZZ}}$ proteins. (A) Mitochondria isolated from cells containing Cox4 $4^{\mathrm{ZZ}}$, Cox $13^{\mathrm{ZZ}}$ and Rcf2 ${ }^{\mathrm{ZZ}}$ were solubilized in $1 \%$ digitonin and subjected to IgG chromatography. Bound proteins were released with SDS sample buffer, and total and eluate fractions were analyzed by SDSPAGE, followed by Western-blotting. (B) $\operatorname{Cox} 4^{\mathrm{ZZ}}$, Cox $13^{\mathrm{ZZ}}$ and Rcf2 $2^{\mathrm{ZZ}}$ complexes were purified from digitonin-solubilized mitochondria via IgG chromatography. Bound complexes were TEV cleaved and analyzed by BN-PAGE and immunodecoration. Total, $10 \%$; eluate $100 \%$.

In addition, the effect of Rcf2 overexpression on respiratory chain supercomplexes was analyzed. Increased levels of Rcf2 had no effect on the steady-state levels of respiratory chain components or control proteins (Figure 3.23A). Upon solubilization of mitochondria in digitonin and BN-PAGE analyses, it could be seen that overexpression of Rcf2 led to a slight increase of supercomplexes (Figure 3.23B). Taken together, this finding together with the previous observation that Rcf2 is not a stoichiometric subunit of supercomplexes, it can be speculated that Rcf2 binds to supercomplexes and facilitates recruitment of some additional proteins. 
A

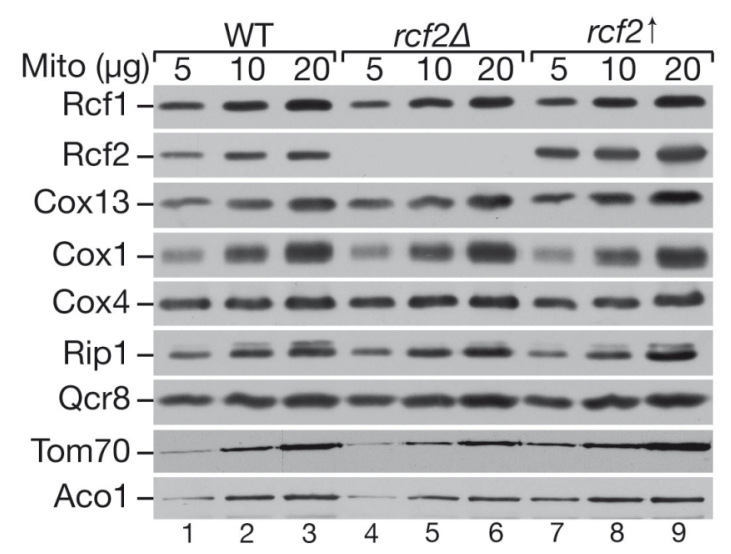

B

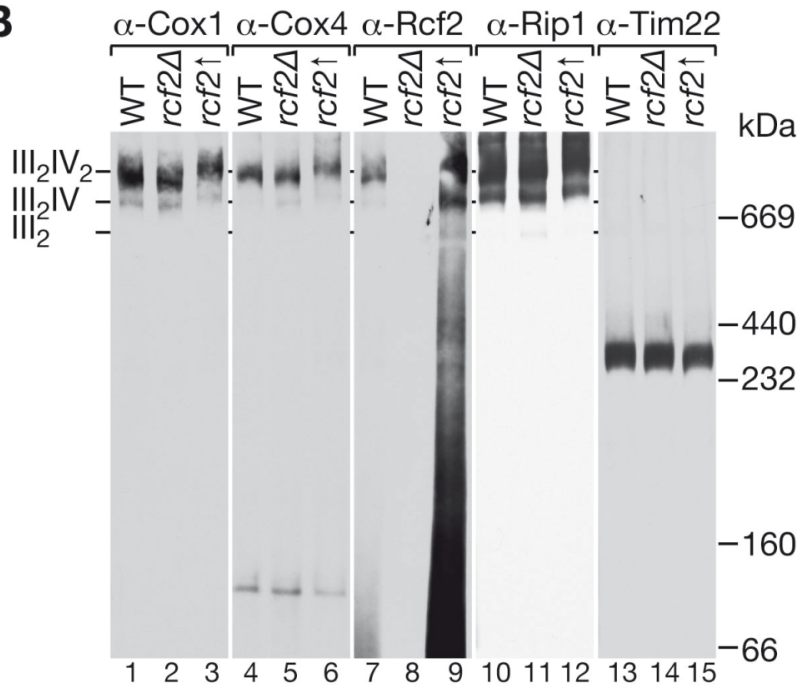

Figure 3. 23 Steady state analysis of mitochondria overexpressing Rcf2. (A) Mitochondria isolated from WT, $r c f 2 \Delta$ and $r c f 2 \uparrow$ strains were analyzed by SDS-PAGE and immunodecoration against indicated proteins. (B) WT, $r c f 2 \Delta$ and $r c f 2 \uparrow$ mitochondria were lysed in $1 \%$ digitonin and analyzed by BN-PAGE and Western-blotting.

\subsection{Distinct forms of cytochrome oxidase exist in mitochondria}

\subsubsection{Purification of complex IV revealed its heterogeneous composition}

The hypothesis that in mitochondria complex IV is present in distinct forms should be confirmed experimentally. To do so, mitochondria containing Cox $4^{\mathrm{ZZ}}$ and $\operatorname{Cox} 13^{\mathrm{ZZ}}$ were solubilized in dodecylmaltoside buffer and $\operatorname{Cox} 4^{\mathrm{ZZ}}$ and $\operatorname{Cox} 13^{\mathrm{ZZ}}$-containing complexes were purified by IgG chromatography. Dodecylmaltoside solubilization of mitochondria disturbs the interactions between complex III and complex IV within supercomplexes and causes their dissociation into a complex IV monomer and a complex III dimer (Schägger and Pfeiffer, 2000). Taking this feature of dodecylmaltoside into account it would be expected to co-isolate with Cox4 and Cox13 only complex IV subunits. Both proteins specifically copurified complex IV, but Cox $13^{\mathrm{ZZ}}$-isolations copurified significantly less complex IV subunits as compared to Cox4 $4^{\mathrm{ZZ}}$-isolations (Figure 3.24A). When unbound fractions of these isolations were 
compared, it was revealed that Cox4 ${ }^{\mathrm{ZZ}}$-isolations were complex IV depleted, whereas this was not the case for Cox $13^{\mathrm{ZZ}}$-isolations (Figure 3.24A, lanes 2 and 3 ). This finding made the previous observation even more definite, suggesting that complex IV differs in regards to Cox13's presence.
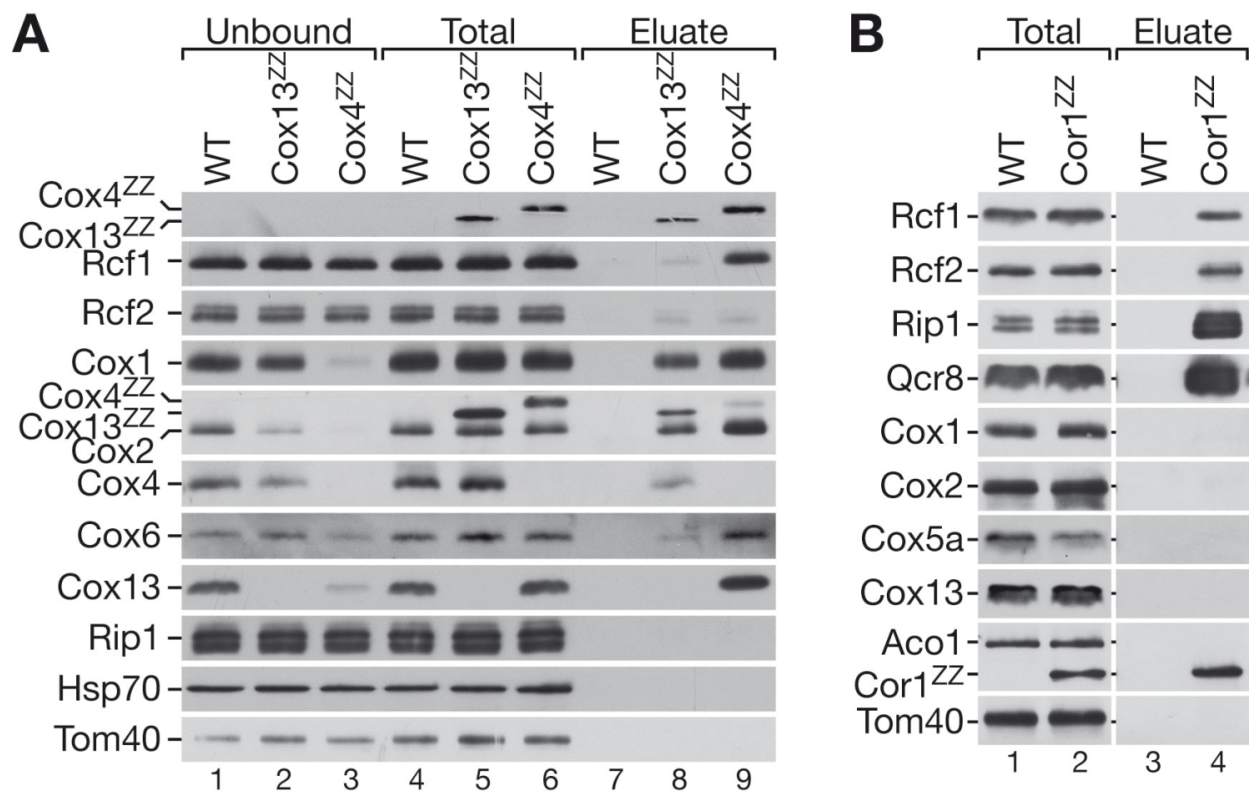

Figure 3. 24 Purification of Cox4 $4^{\mathrm{ZZ}}$, Cox $13^{\mathrm{ZZ}}$ and Cor $1^{\mathrm{ZZ}}$ containing complexes. (A) Mitochondria isolated from indicated strains were solubilized in DDM-containing buffer and subjected to IgG chromatography. Total, eluate and unbound fractions were analyzed by SDS-PAGE and Western-blotting. Total, 25\%; eluate 100\%. (B) WT and Cor $1^{\mathrm{ZZ}}$ mitochondria were treated as in (A). Total, $10 \%$; eluate $100 \%$.

\subsubsection{Rcf1 and Rcf2 can also interact with complex III}

Under the conditions used to solubilize mitochondria in the aforementioned experiment, Rcf1 and Rcf2 dissociated from complex IV. It should be noted that Rcf2 completely dissociated from this complex, whereas a fraction of Rcf1 was detected in Cox4 ${ }^{\mathrm{ZZ}}$-isolations (Figure $3.24 \mathrm{~A}$, lane 9 ). Approximately $20 \%$ of Rcf1 was co-isolated with $\mathrm{Cox} 4^{\mathrm{ZZ}}$.

To purify complex III, mitochondria containing Cor $1^{\mathrm{ZZ}}$ were utilized for solubilization and purification under the same conditions as in the previous 
experiment. Cor $1^{\mathrm{ZZ}}$ purification led to the specific co-isolation of complex III components and small amounts of Rcf1 and Rcf2, but not Cox13 or other complex IV subunits (Figure 3.24B). Roughly $5 \%$ of Rcf1 and Rcf2 were co-purified with Cor $1^{\mathrm{ZZ}}$, indicating that a fraction of these two proteins can associate with complex III. These results suggested that although Rcf1 and Rcf2 preferentially bind to complex IV, they can also interact with complex III and therefore are likely positioned at the interface of complex III/IV oligomers.

\subsubsection{Not all complexes IV posses Cox13}

The finding that only a sub-set of complex IV contains Cox13 implicated that Cox13 is a substoichiometric subunit of this complex and that that complex IV is heterogeneous in nature. This was surprising, because Cox13 was identified in the crystal structure of cytochrome oxidase and was considered to be a structural subunit of that complex (Tsukihara et al., 1996). To assess aforementioned hypothesis experimentally, mitochondria were solubilized in dodecylmaltosidecontaining buffer and analyzed by BN-PAGE and Western-blotting. First, it was observed that dodecylmaltoside solubilization lead to the efficient dissociation of complex $\mathrm{II}_{2}$ from complex IV (Figure 3.25). When the samples were probed for Cox1, a core subunit, it could be detected in two distinct complexes of different size (Figure 3.25, lane 1). These complexes are termed here as IV and IV* and based upon the gel resolution $\mathrm{IV}^{*}$-complexes migrated as either one or two distinct complexes. Although Cox1 was present in both complexes, the amount present in $\mathrm{IV}^{*}$-complexes was significantly less than that in the IV-complex. Contrary to Cox 1 , Cox13 was detected only in $\mathrm{IV}^{*}$-complexes (Figure 3.25 , lane 5 ). When the relative amounts of the two forms, IV and IV ${ }^{*}$, were compared between wild type and $r c f 1 \Delta$ mitochondria, it was observed that the IV-complex levels were similar in both strains, while the amount of the $\mathrm{IV}^{*}$-complex was significantly reduced in $r c f 1 \Delta$. In $\operatorname{cox} 13 \Delta$ and $r c f 2 \Delta$ mitochondria, Cox 1 was detected in both complexes. Thus, the selective lack of complex IV* can be explained by the lack of Rcf1 and not as an indirect consequence of decreased Cox13 levels in $r c f 1 \Delta$ mitochondria, as in the absence of Cox13 Cox1 could be detected in both IV and IV*-complexes (Figure 3.25, lane 4). 


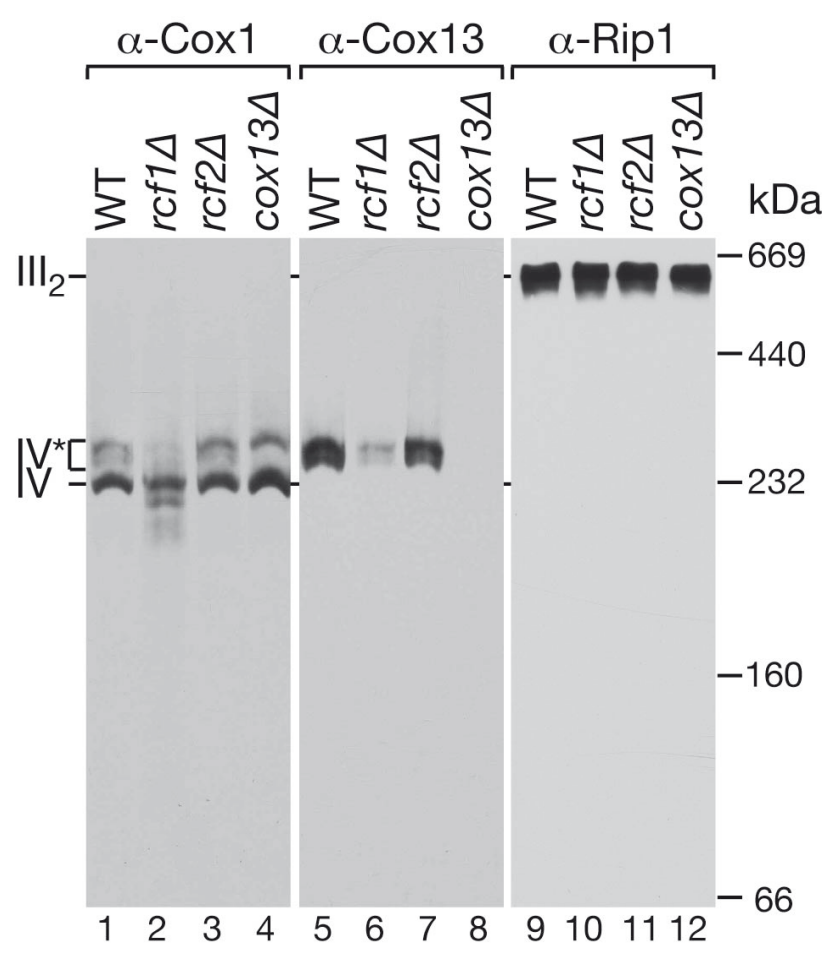

Figure 3. 25 Complex IV exists in distinct forms. Mitochondria isolated from indicated strains were lysed in buffer containing $0.6 \%$ DDM and resulting complexes were analyzed by BN-PAGE and immunodecration for indicated proteins.

If complex IV is heterogenous in regards to its composition, it should be possible to detect the same distinct forms of complex IV when mitochondria are solubilized in other detergents. To test this, mitochondria were solubilized in NP-40 and after BNPAGE analyses, obtained results were similar to those of dodecylmaltoside. Two distinct forms of complex IV were detected, with Cox13 only present in the larger IV*form (Figure 3.26A). Dependent on which detergent was used for solubilization, complex migration distances were different and likely attributed to the various micelle sizes. 

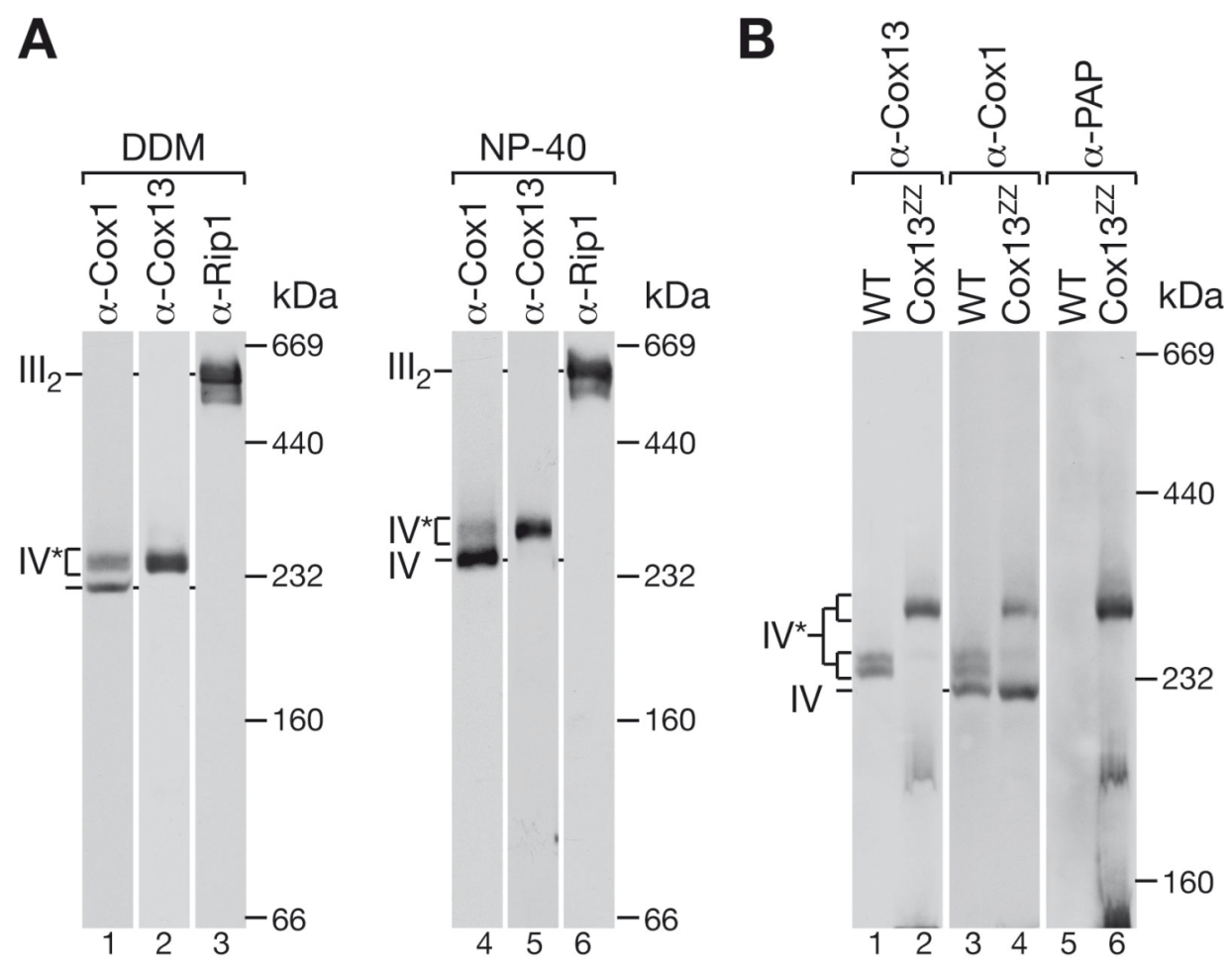

Figure 3. 26 Cox13 is selectively present in complex IV*. (A) WT mitochondria were solubilized in buffer containing $0.6 \%$ DDM or $0.5 \%$ NP-40 and analyzed by BN-PAGE and Western-blotting. (B) Mitochondria isolated from WT and Cox $13^{\mathrm{ZZ}}$ cells were lysed in buffer containing $0.6 \%$ DDM and BN-PAGE analyzed, followed by Western-blotting.

To exclude the possibility that the selective detection of Cox13 in the $\mathrm{IV}^{*}$ complex was due to differential epitope masking between the two complexes, mitochondria expressing ZZ-tagged Cox13 were solubilized in dodecylmaltoside-containing buffer and analyzed by BN-PAGE. Due to the presence of the ZZ-tag on Cox13, the molecular weight of all Cox13-containing complexes should be increased. In comparison with wild type mitochondria, Cox $13^{\mathrm{ZZ}}$ mitochondria displayed a specific mass-shift of complex IV*, whereas complex IV was not affected (Figure 3.26B). This finding strongly supports the previous conclusion that Cox13 is selectively present in complex IV*. Thus, Cox13 can be characterized as a constituent of a defined subclass of cytochrome oxidase complexes in mitochondria, of which formation is largely Rcf1 dependent. 


\section{Discussion}

Oligomerization of respiratory chain complexes into supercomplexes or respirasomes has been verified by various approaches (Schägger and Pfeiffer, 2000; Cruciat et al., 2000; Dudkina et al., 2005; Heinemeyer et al., 2007; Acin-Perez et al., 2008). The cellular significance of these structures was explained by several different hypotheses (Schägger, 2002; Schägger et al., 2004; Moran et al., 2010). However, experimental verification of these ideas is still lacking, mainly because of the void of established factors, which facilitate supercomplex formation and stabilization. Also, several recent studies have reported that supercomplexes could interact with a diverse array of accessory factors (van der Laan et al., 2006; Wiedemann et al., 2007; Mick et al., 2007; Saddar et al., 2008; Dienhart and Stuart, 2008; Mick et al., 2010), hindering the identification of oligomerization factors.

\subsection{Identification of supercomplex interacting components}

The first aim of this study was to search for new respirasome components that could be designated either as oligomerization factors or as supercomplex associated factors. Mass spectrometric analysis of proteins copurified with yeast TAP tagged Cor1 protein revealed Rcf1 and Rcf2 as new subunits of supercomplexes. Both proteins are located in the inner mitochondrial membrane with their C-terminal domains exposed to the intermembrane space. While Rcf2 is specific to yeast, Rcf1 is conserved among eukaryotes and has two human orthologs, RCF1a and RCF1b. Both proteins, Rcf1 and Rcf2, are characterized as cytochrome oxidase components. In fact, binding of Rcf2 to the cytochrome oxidase is dependent on Rcf1, which is responsible for supercomplex assembly and stability.

In addition to these two proteins, several others were found to co-isolate with purified supercomplexes. Although the co-purification of Fmp25 and Cox26 were recognized as specific, attempts to verifiy their mitochondrial localization and association to supercomplexes failed. Initial radioactive imports of these two proteins into isolated mitochondria were attempted, however, the inability to detect mitochondrially located radioactive protein excluded these two candidates from the current study. 
Nevertheless, Fmp25 and Cox26 were still reproducible shown to specifically associate with respiratory supercomplexes in mass spectrometry analyses. Indeed, in an independent study Fmp25 was characterized as an assembly factor of complex III (Mathieu et al., 2011). The fact that complex III and IV subassemblies can bind to the supercomlexes (Fernandez-Vizzara et al., 2009; Mick et al., 2007) explains the presence of this protein within respirasomes. The inability to show mitochondrial import of the radio-labelled protein could be explained simply by the lack of an essential factor in the in vitro setting, which would cause folding or aggregation of hydrophobic protein. Alternatively, and most likely, the cleavage of the presequence upon import resulting in the loss of the radiolabel due to the lack of downstream methionine residues would explain the failure to detect a processed protein form in the import experiments.

Mio10 was identified in all supercomplex purification replicates, but its enrichment was not sufficient to consider it as a specific interaction partner of supercomplexes although Mio10's mitochondrial localization has been established. Due to this lack of purification efficiency its further characterization was excluded from this study. Recent results from the work of our and several independent groups reported on the involvement of this protein in the formation of the MINOS complex responsible for the formation of cristae junctions (von der Malsburg et al., 2011; Alkhaja et al., 2012; Hoppins et al., 2011; Harner et al., 2011). None of the other MINOS complex components were copurified with supercomplexes, speaking against the possibility that MINOS and supercomplexes interact, which would suggest that Mio10 possess dual functions. Interestingly, purification of the MINOS complex from isolated mitochondria lead to the co-isolation of Rcf2 (von der Malsburg et al., 2011), which was shown to facilitate the recruitment of additional proteins to supercomplexes. Therefore, an alternative function of Mio10 could be related to supercomplexes via Rcf2.

\subsection{Rcf1 is a conserved component of complex IV}

Rcf1 and Rcf2 have been identified as interaction partners of supercomplexes and subsequent analyses assigned them as novel constituents of complex IV. Also, both 
human homologs of Rcf1, RCF1a and RCF1b, were found to associate with complex IV. Although it has been shown that the function of Rcf1 is to promote supercomplex association and stability, in the absence of Rcf1 a complete dissociation of supercomplexes was not observed, but rather a partial dissociation. This result is explained by the presence of a heterogenous cytochrome oxidase population that differs in the presence or absence of Cox13 (homolog of human COX6a). It has been demonstrated that Cox13 is present in only certain cytochrome oxidase complexes, and that the majority of these complexes do not contain Cox13. In spite of the fact that Rcf1 is conserved component of the cytochrome oxidase, this protein was not identified in the crystal structure (Tsukihara et al., 1996). In mitochondria lacking Rcf1 Cox13 is still present in respiratory complexes, indicating that cytochrome oxidase complexes not only differ with regard to the presence or absence of Cox13, but also in regards to the presence of Rcf1 amongst the Cox13containing population. Thus, one could speculate that only the Cox13 containing complex IV form was isolated and crystallized, therefore explaining the lack of Rcf1 in the current structure. On the other hand, method employed for the analyses of the crystal structure of cytochrome oxidase did not involve affinity purification of cytochrome oxidase, but salting out of the complex from mitochondria extracted with sodium cholate (Yoshikawa et al., 1977; Tsukihara et al., 1996). As seen in this study, mitochondrial solubilization in any non-ionic detergent stronger than digitonin results in the dissociation of Rcf1, therefore explaining the absence of Rcf1 from the crystal structure due to the stringent precipitation method.

\subsection{Rcf1 requirement for complex IV activity is coupled to Cox13 function}

Assaying growth behavior of yeast cells lacking Rcf1 showed that this protein is necessary for efficient growth on non-fermentable carbon sources. This mutant is able to grow on non-fermentable carbon sources, but the growth rate is reduced in comparison to wild type cells, indicating a functional role of the protein within the respiratory chain. This was confirmed by showing that Rcf1 is required for cytochrome oxidase activity. Cytochrome oxidase activity was reduced in $r c f 1 \Delta$ 
mitochondria and the extent of the reduction resembles that of mitochondria lacking

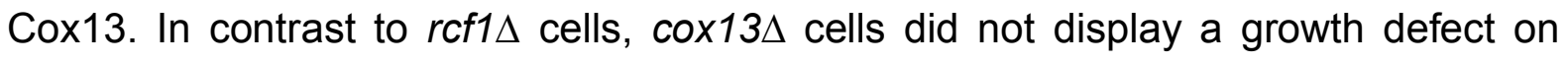
non-fermentable carbon sources. Therefore, the growth defect observed in $r c f 1 \Delta$ cells is not simply a consequence of reduced cytochrome oxidase activity.

Although Cox13 was identified in the crystal structure of bovine cytochrome oxidase (Tsukihara et al., 1996), and thus designated as a structural subunit, it does not affect the stability of the enzyme (Taanman and Capaldi, 1993). However, the evolutionary conservation of Cox13 raised the idea that this protein is important for the enzymes functionality. The exact function of Cox13 has yet to be determined, but it might modulate the activity of cytochrome oxidase in an ATP-dependent manner (Taanman and Capaldi, 1993). The human homolog of Cox13, COX6a, displays tissue-specific isoforms (Mell et al., 1994) and it was reported that the COX6a isoform expressed in heart tissue $(\mathrm{COX} 6 \mathrm{a}-\mathrm{H})$ could interact with matrix ADP, thus regulating cytochrome oxidase activity (Anthony et al., 1993). Therefore, this protein might regulate respiratory rate through the inhibition or stimulation of cytochrome oxidase activity under various conditions. As reported in this study, the stability and efficient assembly of Cox13 are dependent on Rcf1. Hence, it is likely that the observed defect in cytochrome oxidase activity in $r c f 1 \Delta$ mitochondria is coupled to the function of Cox13.

\subsection{Rcf1 facilitates supercomplex formation in hypoxia}

This study shows that the growth defect in $r c f 1 \Delta$ cells is exacerbated in conditions where oxygen concentrations are limiting, suggesting the importance of Rcf1 under hypoxic conditions. It was shown that mitochondria from yeast cells grown on nonfermentable carbon sources contain higher amounts of respiratory complexes compared to those grown on fermentable carbon sources, additionally the ratios of different supercomplex forms varies with regard to growth conditions (Schägger and Pfeiffer, 2000; Pfeiffer et al, 2003). In line with these observations, it can be hypothesized that supercomplexes are responsible for the fine-tuning of cell growth behavior in response to different environmental conditions. Respiratory chain function must meet the energy demands of the cell under non-fermentable growth 
conditions. As the ratio of different supercomplex forms changes with regard to environmental carbon sources, it is tempting to speculate that a similar regulatory system is at play in regards to local oxygen concentration. In this case, supercomplexes should not be viewed as a rigid higher-ordered assembly (Schägger and Pfeiffer, 2000), but rather as a dynamic system that can reorganize in response to different stimuli (Acin-Perez et al., 2008) and therefore provide conditions optimal for cell growth tailored to the local environment. Given that the function of Rcf1 is to stabilize supercomplexes, one could speculate that Rcf1 assists in the reorganization of supercomplexes. Also, it has been shown that mouse homolog of Rcf1 is robustly induced in hypoxia (Denko et al., 2000). Therefore, in the absence of Rcf1 proposed supercomplex reorganization is prohibited and cells are not able to develop optimally under low oxygen conditions.

\subsection{Rcf1 association with complex III}

Rcf1 was designated as a novel complex IV component primarily based on the assembly and activity results in isolated mitochondria from $r c f 1 \Delta$ cells. Since the absence of this protein affects the stability of supercomplexes, it is proposed that Rcf1 is a complex IV subunit with affinity to bind complex III and thus promote supercomplex formation (Figure 4.1A). When supercomplexes were disturbed by dodecylmaltoside treatment, Rcf1 as well as Rcf2 remained associated with either complex III or IV. However, the interactions with complex III are significantly weakened in comparison to that with complex IV. As Rcf1 functions in a manner to stabilize supercomplexes, it is likely that it is positioned close to the interface between complexes III and IV, thus providing a physical link between the two complexes. Due to its proposed function, Rcf1 must have affinity for both complexes. Two independent studies provided data to support this notion. Purification of Rcf1 from mitochondria solubilized with dodecylmaltoside resulted in the specific coisolation of complex IV components (Chen et al., 2012; Strogolova et al., 2012). However, when Rcf1 was immunoprecipitated from different mutants of compromised complex IV formation its interaction was shifted to complex III (Chen et al., 2012). This raised an alternative hypothesis that Rcf1 is actually the 
oligomerization factor able to interact with both complexes independently (Figure 4.1B). The aforementioned observation that Rcf1 retained bound to both complex III and IV when supercomplexes are dissociated with dodecylmaltoside is in line with its role as an oligomerization factor, but to establish its definitive function requires further more intricate analyses.

A

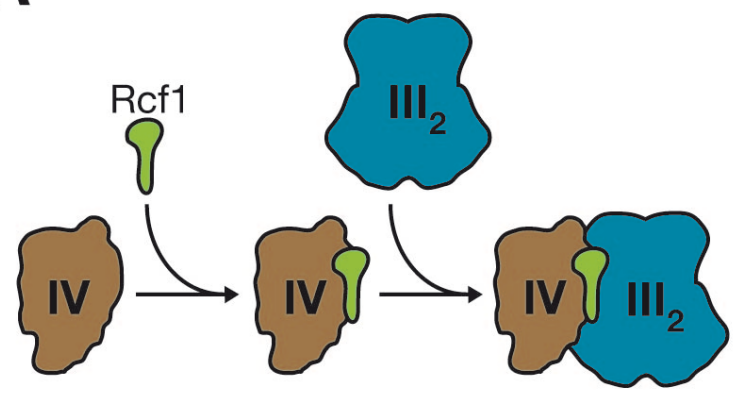

B

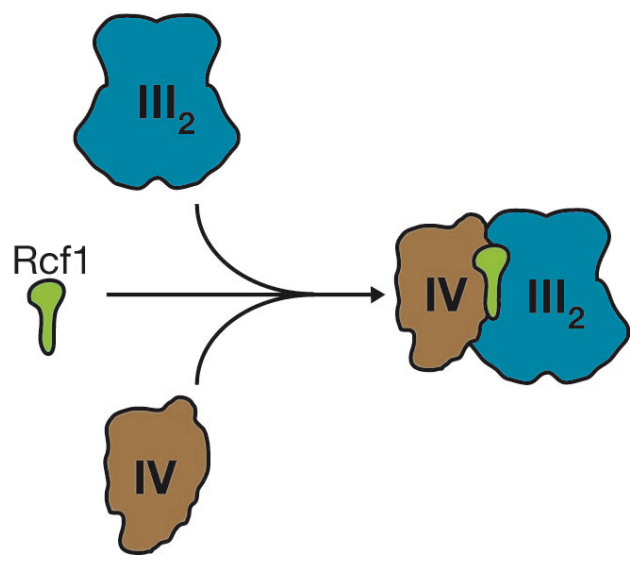

Figure 4. 1 Rcf1 facilitates interaction of complex $\mathrm{III}_{2}$ and IV. (A) Rcf1 binds to previously assembled complex IV and due to its affinity for complex III promotes binding of complex $\mathrm{III}_{2}$ to complex IV. (B) Interaction of complex $\mathrm{III}_{2}$ and IV occurs in the presence of Rcf1 that is able to bind any of these two complexes.

\subsection{Rcf1 acts at the interface of complex III and IV}

The recently published 3D high-resolution electron microscopy reconstruction of the bovine $I_{1} I I_{2} I V_{1}$ respirasome provides insight into the interaction between single complexes within this structure (Dudkina et al., 2011; Althoff et al., 2011). According to these data, complex IV interfaces with complex III through Cox3, Cox7 and Cox13. Since Rcf1 is necessary for efficient assembly of Cox13, it is likely that Cox13 and Rcf1 are in close proximity, which places Rcf1 at the interface of complex III and IV, allowing for its interaction with both complexes. This idea is further 
supported by the observation that Rcf1 specifically interacts with Cox3, located at the complex III/IV interface. In different complex IV assembly mutants the interaction of Rcf1 with Cox3 was unaffected (Chen et al., 2012). Such an observation can be explained by the fact that Rcf1 and Cox3 are in close proximity. The unaltered interaction of Rcf1 with Cox3 in the absence of functional complex IV is difficult to explain, largely due to the void of information regarding supercomplex formation. It is believed that each complex follows an independent biosynthetic pathway and when individual complexes $\mathrm{III}_{2}$ and IV accumulate above certain threshold (D'Aurelio et al., 2006; Acin-Perez et al., 2008) these pathways converge, which results in the association of preassembled complexes into higher-ordered structures. This process is at least in part regulated by Rcf1, which is proposed to bind to mature complex IV. Therefore, the binding of Rcf1 occurs upon the biogenesis of the complex core and incorporation of most of the structural subunits. Moreover, it is to be expected that mutations that impair complex IV assembly also affect Rcf1's complex association, which would imply the absence of Rcf1's interaction to all of the cytochrome oxidase subunits.

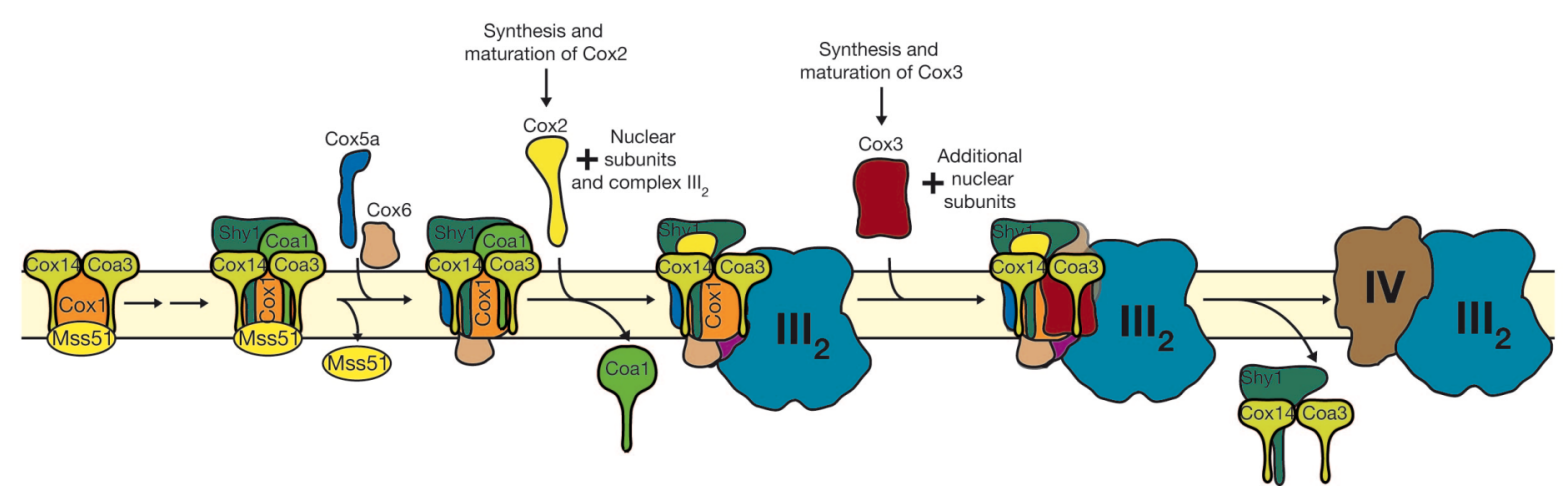

Figure 4. 2 Model for complex IV assembly. Upon synthesis and membrane insertion of Cox1is bound to translational regulator complex of Mss51, Cox14 and Coa3. This is one of the first cytochrome oxidase assembly intermediates. In the following assembly steps assembly factors, Coa1 and Shy 1 bind to this complex. Through the incorporation of subunits (e.g. Cox5a and Cox6), complex IV subcomplexes are formed and Mss51 is released from the subassembly. As more subunits such as Cox2, get incorporated, Coa1 is released. Partially assembled complex IV, still containing translational activators and assembly factors is able to associate to complex $\mathrm{II}_{2}$. Late steps of complex IV assembly may take place at the supercomplex level in the presence of complex $\mathrm{II}_{2}$ and upon formation of holo-complex IV translational regulators and assembly factors are released from the complex. 
On the other hand, biogenesis of the supercomplexes can be viewed as a multistep process in which individual subunits or subassemblies of the single complexes are sequentially added. Such a model has been recently proposed and is based on the assembly kinetic of respiratory chain subunits in mammalian cells (Moreno-Lastres et al., 2012). According to this model, the biosynthesis of complexes $\mathrm{III}_{2}$ and IV occurs through several independent pathways, and can assemble either as individual enzymes or by the binding of subunits and subassemblies to a supercomplex assembly intermediate, formed around a complex I intermediate lacking the catalytic module. Although the process of cytochrome oxidase assembly is not yet solved, it is known that the assembly starts with Cox1 and continues with the sequential addition of other subunits and formation of different assembly intermediates (Figure 4.2) (Nijtmans et al., 1998; Horan et al., 2005; Fontanesi et al., 2006). Furthermore the biogenesis of the core components, Cox 1 , Cox2 and Cox3, follow relatively independent paths and their incorporation takes place at different stages of complex formation (Figure 4.2) (Herrmann et al., 2005; Soto et al., 2011). Also, cytochrome oxidase subassemblies are able to interact with other respiratory chain components at early stages of supercomplex assembly (Figure 4.2) (Mick et al., 2007; Mick et al., 2010). Taking this into account, one could speculate that certain populations of complex IV are formed by direct binding of subunits and subassemblies to the complex III dimer, which serves as a scaffold for complex IV assembly (Figure 4.3). In this case, Rcf1 can associate directly to supercomplex prior to the formation of holo-complex IV or can interact with some of the complex IV subunits and subsequently assist in complex $\mathrm{III}_{2}$ association due to its affinity for complex III (Figure 4.3). Although in this model the association of some cytochrome oxidase subunits to complex $\mathrm{III}_{2}$ occurs prior to complex IV binding, it is consistent with the proposed cytochrome oxidase assembly scheme as it commences with Cox1 maturation (Nijtmans et al., 1998) and it is in agreement with the observation that newly imported nuclear-coded complex IV components could bind directly to supercomplexes in the absence of monomeric complex IV (Lazarou et al., 2009). 


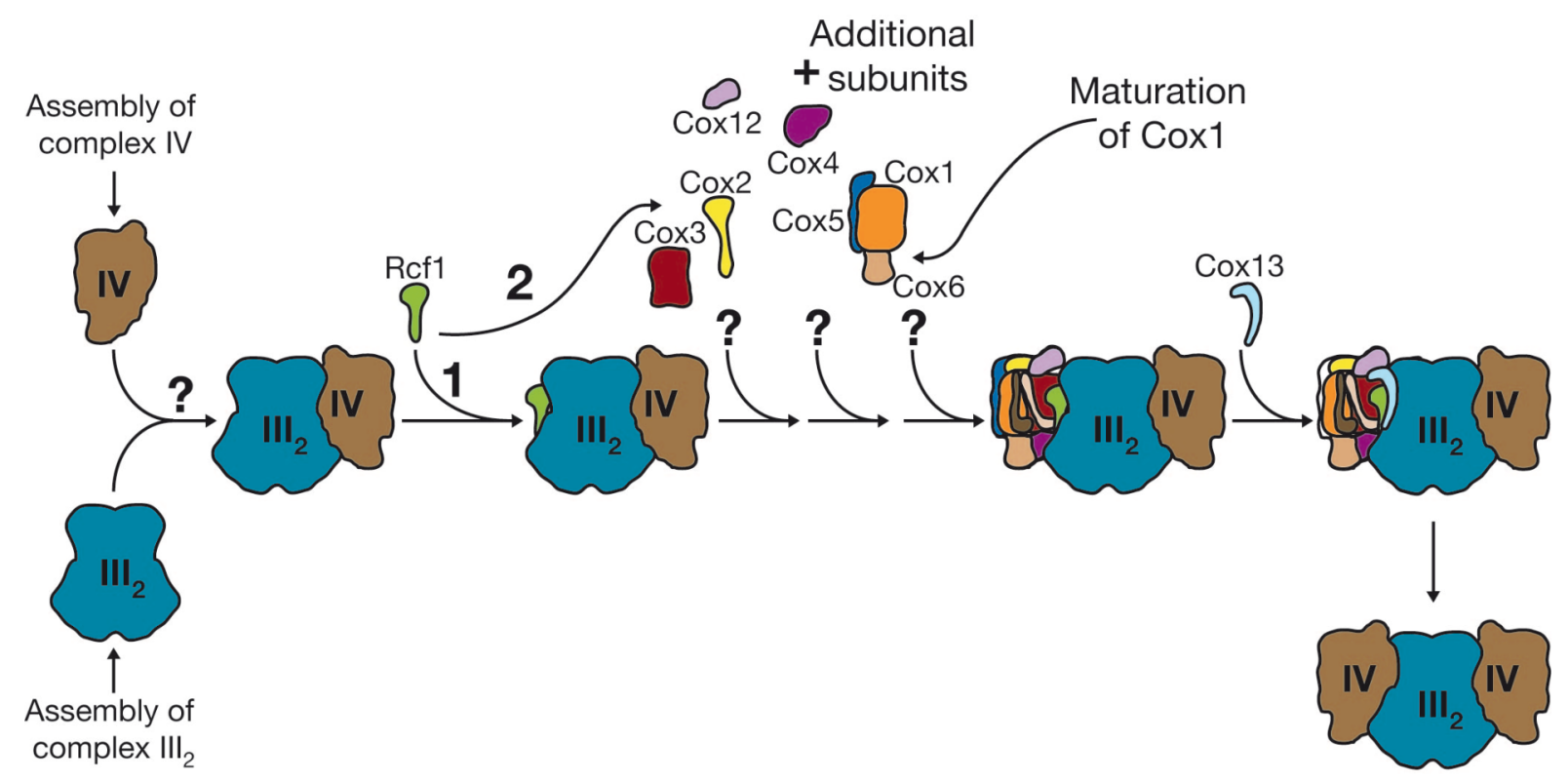

Figure 4. 3 Model for the assembly of mitochondrial respiratory supercomplexes. In the first stage, preassembled complexes $\mathrm{II}_{2}$ and IV associate into supercomplex $\mathrm{III}_{2} \mathrm{IV}$ independently of Rcf1. This is a platform for the subsequent binding of subunits and subassemblies of complex IV, which leads to the generation of supercomplex $\mathrm{II}_{2} \mathrm{IV}_{2}$. This process depends on Rcf1. Incorporation of Rcf1 can follow one of the two paths; either 1 in which it incorporates before other subunits, or 2 where it assembles together with other subunits of complex IV. The latest step involves the association of Cox13.

Viewing supercomplex formation in this way would explain why the integration of Cox13 in certain complexes requires Rcf1. A scenario in which the assembly of complex IV takes place on complex $\mathrm{III}_{2}$ predicts that Rcf1 binds to the complex prior to Cox13 incorporation into holo-complex IV and directly affects Cox13's complex affinity. If Cox13 is a functional subunit that regulates the activity of the cytochrome oxidase (Taanman and Capaldi, 1993; Anthony et al., 1993), then Rcf1's alteration of Cox13's complex affinity is very likely. Under conditions when the function of the respiratory chain needs to be tightly regulated, Rcf1 could serve to increase the association of Cox13 with complex IV, providing more efficient complex activity control.

Considering the fact that in mitochondria isolated from $r c f 1 \Delta$ cells $\mathrm{III}_{2} \mathrm{IV}_{2}$ supercomplexes are specifically affected, it is likely that Rcf1 serves to link the second monomer of complex IV with the $\mathrm{III}_{2} \mathrm{IV}$ form. When the results of this study in 
conjunction with the established literature is taking into account, it is possible to present a model (Figure 4.3) that considers respirasomes as a flexible system, sensitive to various environmental stimuli through the regulation of ratio of different forms of the supercomplexes in order to provide them to function the most efficiently under given conditions. Rcf1 is considered to be the main factor responsible for these adjustments. As rcf1 $\Delta$ cells displayed a more severe phenotype under hypoxia, it is likely that Rcf1 is tightly regulated by different oxygen concentrations, which agrees with the observation that in mouse expression of Rcf1 homolog is induced by hypoxia (Denko et al., 2000). In this model the biogenesis of supercomplexes follows two alternative pathways, and the formation of two oligomers, $\mathrm{II}_{2} \mathrm{IV}$ and $\mathrm{II}_{2} \mathrm{IV} \mathrm{V}_{2}$, differ. Complexes $\mathrm{II}_{2}$ and IV assemble as individual entities and upon completion they interact independently of Rcf1 and form the $\mathrm{II}_{2} \mathrm{IV}$ supercomplex (Figure 4.3). The construction of the $\mathrm{III}_{2} \mathrm{IV}$ 年 supercomplex is achieved through an alternative pathway, in which complex $\mathrm{III}_{2} \mathrm{IV}$ serves as a scaffold for the assembly of the second complex IV subunit in an Rcf1 dependent manner (Figure 4.3). The generation of the $\mathrm{III}_{2} I V_{2}$ complex is favored in conditions where more tight control of the respiratory chain function is required. This modulation is carried out by Rcf1's ability to alter Cox13's complex binding affinity. Therefore, Rcf1 is a factor that directly mediates respirasome formation, but its effect on the function of the respiratory chain is indirect.

In summary, this model is in line with the observations seen in this study in regards to the function of Rcf1 and does not necessarily contradict the notion that Rcf1 is a cytochrome oxidase component.

As demonstrated in this study, complex IV exists in different isoforms, with the IV* isoform containing Cox13. As this complex is almost completely absent in rcf1 mitochondria it is likely that Rcf1 is necessary for its formation and that the majority of Cox13 and Rcf1 are in the same complex. It can be reasoned that complex IV* is actually the isoform capable of binding to $\mathrm{II}_{2} \mathrm{IV}$. However, this cannot explain the fact that in mitochondria isolated from $r c f 1 \Delta$ cells the $I I I_{2} I V_{2}$ supercomplexes are significantly affected, due to the fact that complex IV* represents a less abundant cytochrome oxidase form. Thus, it appears that Rcf1 is responsible for the 
recruitment of complex $\mathrm{IV}^{*}$ as well as complex IV to respiratory chain supercomplexes.

\subsection{Implication of complex IV* and Rcf1/2 in ROS production}

While Rcf1 deficiency leads to a variety of defects, $r c f 2 \Delta$ cells did not share any of these phenotypes. The only abnormality observed in the mitchondria of $r c f 2 \Delta$ cells was the increase in ROS production, as seen in mitochondria isolated from $r c f 1 \Delta$ and cox13 $\Delta$ cells. The phenotype observed in $r c f 1 \Delta$ mutants resembled that of $r c f 2 \Delta$, while the phenotype of cox13 13 mitochondria was rather moderate. As cytochrome oxidase activity was not impaired in mitochondria lacking Rcf2 and ROS production in $\operatorname{cox} 1^{-}$-mitochondria was not increased in comparison to wild type, the increase in ROS production is likely not related to cytochrome oxidase dysfunction. Additionally, the selective growth defect observed in the $r c f 1 \Delta$ mutants cannot be explained by increased ROS production, because $r c f 2 \Delta$ cells grow normally in comparison to wild type. Due to the fact that the assembly of Cox13 and Rcf2 require Rcf1 to a large extent, and that Cox13 is required for the assembly of Rcf2, it appears that all three proteins are present in the same cytochrome oxidase complex. Being that complex $\mathrm{IV}^{*}$ is almost completely absent in $\operatorname{rcf} 1 \Delta$ mitochondria it is tempting to speculate that these proteins are constituents of this complex. Although the current study did not provide experimental verification of this assumption, mainly because Rcf1 and Rcf2 dissociate under conditions in which complex IV* can be analyzed, it is possible that complex IV* plays an important role in the protection of the respiratory chain from ROS generation. Therefore, the absence of any of these subunits can affect mitochondrial function and consequently increased ROS production. However, experimental evidence in direct support of this model is not yet available, but should commence by establishing the conditions enabling the dissection of the $\mathrm{IV}^{*}$ isoform of complex IV.

\subsection{Functional conservation of Rcf1 in mammals}

As Rcf1 shows evolutionary conservation, it was of interest to determine whether its human homologs play a similar role. Unfortunately, knockdown of neither of its two 
human homologues, RCF1a and RCF1b, in mammalian HEK293 cells had an effect on supercomplex assembly. However, the inability to detect a phenotype in mammalian cells does not exclude the possibility that the function of Rcf1 seen in yeast is actually conserved. A likely explanation for this discrepancy is that the transfection conditions and/or incubation time with the siRNA were not optimal for the given cell type. Thus, it will be of great interest to optimize these parameters for the specific cells used as well as to analyse the effect of silencing these two genes in different cell lines. With regard to functional conservation of Rcf1 in supercomplex organization, it was demonstrated that the treatment of mouse cells with siRNA against RCF1b causes supercomplex depletion (Chen et al., 2012). 


\subsection{Final remarks and outlook}

The identification of Rcf1 as a respiratory supercomplex assembly and stability regulator is a breakthrough in the understanding of the role and relevance of supercomplexes. However, it is just the tip of the iceberg, therefore it is of the utmost importance to look what lies beneath in order to obtain a complete understanding of supercomplexes. There are numerous questions with regard to respirasomes. How are they formed? How is the process of their formation is regulated? What are the factors involved in their assembly and stability? What are the consequences of their disassembly? How compositionally diverse are supercomplexes? Based on the available data, various hypotheses on the importance of respiratory chain oligmerization were proposed, but the experimental verification of these models is a yet insurmountable task. One of the main reasons for the lack of respiratory oligomerization mutants is the global severity of supercomplex formation upon gene deletion. In this study, the deletion of RCF1 is shown to cause the specific dissociation of supercomplexes in yeast, and therefore enable the genetic manipulation of supercomplex formation allowing for subsequent investigations to probe their function and assembly. The fact that Rcf1 is conserved and possess homologs in various organisms extends research relevance to higher eukaryotes, in particular mammals. Therefore, RCF1 presents a valuable tool for the investigation of the function of respiratory supercomplexes in the context of human health and disease.

Considering that Rcf1 itself is of great importance for analysing its regulation in different oxygen conditions, one should consider whether the transcription of Rcf1 is regulated by changes in oxygen concentrations or whether the regulation takes place at the post-translational level. If the latter is true, it will be important to reveal the mechanism responsible for this regulation. Is this the only regulation of Rcf1 or is the stability of the protein regulated by oxygen availability? Moreover, the role of Rcf1 needs to be elucidated, and for this it will be of great importance to clearly define if this protein is a cytochrome oxidase subunit. One potential strategy would be to generate mutant strains that are unable to form complex III, but contain a functional 
tag on complex IV and vice versa, which will allow for the specific isolation of either complex IV or complex III. Resolving the aforementioned aspects of Rcf1 will help to answer the numerous outstanding questions. 


\section{Summary}

The mitochondrial respiratory chain consists of four membrane-embedded electron transport complexes (complexes I - IV) and the ATP synthesizing $F_{1} F_{0}$ ATPase. Within the inner mitochondrial membrane these complexes assemble into supercomplexes, forming so-called respirasomes. Although understanding the biogenesis and function of these higher oligomeric structures has been the subject of intense research for many years it still remains largely enigmatic. The aim of this work was to obtain novel insights into respirasome formation and their cellular relevance.

Using an affinity chromatography based strategy respiratory supercomplexes from yeast, S. cerevisiae mitochondria were purified and novel factors identified and characterized. Rcf1 and Rcf2 are reported to be complex IV components. While Rcf2 is present only among the yeast species, Rcf1 is a conserved subunit and has two human homologs, RCF1a and RCF1b. Yeast cells lacking Rcf1 are affected in cytochrome oxidase activity and display reduced growth efficiency, especially pronounced during hypoxia. Both mutants, $r c f 1 \Delta$ and $r c f 2 \Delta$, show increased ROS production. Rcf1 mediates oligomerization of respiratory complexes, specifically for the formation of oligomers consisting of complex III dimer and two complex IV monomers. Additionally, Rcf1 is necessary for efficient assembly of subunits Cox 13 and Rcf2 into the cytochrome oxidase complex.

The identification of Rcf1 revealed that mitochondria possess a heterogenous cytochrome oxidase population. The various forms of cytochrome oxidase complexes differ in their subunit composition. At least two cytochrome oxidase subpopulations can be distinguished, one containing Cox13 and one that lacks the Cox13 subunit. Selective loss of the Cox13-containing form was observed in rcf1 $\Delta$ mitochondria. Both forms are able to incorporate into supercomplexes, thus generating distinct forms of supercomplexes. 


\section{References}

Acin-Perez, R., Bayona-Bafaluy, M. P., Fernandez-Silva, P., Moreno-Loshuertos, R., Perez-Martos, A., Bruno, C., Moraes, C. T., Enriquez, J. A. (2004) Respiratory complex III is required to maintain complex I in mammalian mitochondria, Mol Cell 13:805-15

Acin-Perez, R., Fernandez-Silva, P., Peleato, M. L., Perez-Martos, A., Enriquez, J. A. (2008) Respiratory active mitochondrial supercomplexes, Mol Cell 32:529-39

Ahting, U., Thieffry, M., Engelhardt, H., Hegerl, R., Neupert, W., Nussberger, S. (2001) Tom40, the pore-forming component of the protein-conducting TOM channel in the outer membrane of mitochondria, J Cell Biol 153:115160

Alkhaja, A. K., Jans, D. C., Nikolov, M., Vukotic, M., Lytovchenko, O., Ludewig, F., Schliebs, W., Riedel, D., Urlaub, H., Jakobs, S., Deckers, M. (2012) MINOS1 is a conserved component of mitofilin complexes and required for mitochondrial function and cristae organization, Mol Biol Cell 23: 247-57

Allen, L. A., Zhao, X. J., Caughey, W., Poyton, R. O. (1995) Isoforms of yeast cytochrome c oxidase subunit V affect the binuclear reaction center and alter the kinetics of interaction with the isoforms of yeast cytochrome $\mathrm{c}, \mathrm{J}$ Biol Chem 270: 110-8

Altamura, N., Capitanio, N., Bonnefoy, N., Papa, S., Dujardin, G. (1996) The Saccharomyces cerevisiae OXA1 gene is required for the correct assembly of cytochrome c oxidase and oligomycin-sensitive ATP synthase, FEBS Lett 382: 111-5

Althoff, T., Mills, D. J., Popot, J. L., Kuhlbrandt, W. (2011) Arrangement of electron transport chain components in bovine mitochondrial supercomplex I1III2IV1, EMBO J 30: 4652-64

Anthony, G., Reimann, A., Kadenbach, B. (1993) Tissue-specific regulation of bovine heart cytochrome-c oxidase activity by ADP via interaction with subunit Vla, Proc Natl Acad Sci U S A 90:1652-6

Arnold, I., Pfeiffer, K., Neupert, W., Stuart, R. A., Schagger, H. (1998) Yeast mitochondrial F1F0-ATP synthase exists as a dimer: identification of three dimer-specific subunits, EMBO J 17:7170-8

Arselin, G., Vaillier, J., Salin, B., Schaeffer, J., Giraud, M. F. Dautant, A., Brethes, D., Velours, J. (2004) The modulation in subunits e and $\mathrm{g}$ amounts of yeast ATP synthase modifies mitochondrial cristae morphology, $\mathrm{J}$ Biol Chem 279:40392-9

Barrientos, A., Fontanesi, F., Diaz, F. (2009) Evaluation of the mitochondrial respiratory chain and oxidative phosphorylation system using polarography and spectrophotometric enzyme assays, Curr Protoc Hum Genet Chapter 19:Unit19 3

Barrientos, A., Zambrano, A., Tzagoloff, A. (2004) Mss51p and Cox14p jointly regulate mitochondrial Cox1p expression in Saccharomyces cerevisiae, EMBO J 23:3472-82

Barros, M. H., Carlson, C. G., Glerum, D. M., Tzagoloff, A. (2001) Involvement of mitochondrial ferredoxin and Cox15p in hydroxylation of heme O, FEBS Lett 492:133-8

Barros, M. H., Tzagoloff, A. (2002) Regulation of the heme A biosynthetic pathway in Saccharomyces cerevisiae, FEBS Lett 516:119-23

Becker, L., Bannwarth, M., Meisinger, C., Hill, K., Model, K., Krimmer, T., Casadio, R., Truscott, K. N., Schulz, G. E., Pfanner, N., Wagner, R. (2005) Preprotein translocase of the outer mitochondrial membrane: reconstituted Tom40 forms a characteristic TOM pore, J Mol Biol 353:1011-20

Becker, T., Bottinger, L., Pfanner, N. (2012) Mitochondrial protein import: from transport pathways to an integrated network, Trends Biochem Sci 37:85-91

Belevich, I., Verkhovsky, M. I., Wikstrom, M. (2006) Proton-coupled electron transfer drives the proton pump of cytochrome c oxidase, Nature 440:829-32 
Boumans, H., Grivell, L. A., Berden, J. A. (1998) The respiratory chain in yeast behaves as a single functional unit, J Biol Chem 273:4872-7

Brandner, K., Mick, D. U., Frazier, A. E., Taylor, R. D., Meisinger, C., Rehling, P. (2005) Taz1, an outer mitochondrial membrane protein, affects stability and assembly of inner membrane protein complexes: implications for Barth Syndrome, Mol Biol Cell 16: 5202-14

Brandt, U., Yu, L., Yu, C. A., Trumpower, B. L. (1993) The mitochondrial targeting presequence of the Rieske iron-sulfur protein is processed in a single step after insertion into the cytochrome bc1 complex in mammals and retained as a subunit in the complex, J Biol Chem 268:8387-90

Brix, J., Dietmeier, K., Pfanner, N. (1997) Differential recognition of preproteins by the purified cytosolic domains of the mitochondrial import receptors Tom20, Tom22, and Tom70, J Biol Chem 272:20730-5

Bunn, H. F., Poyton, R. O. (1996) Oxygen sensing and molecular adaptation to hypoxia, Physiol Rev 76:839-85

Burke, P. V., Poyton, R. O. (1998) Structure/function of oxygen-regulated isoforms in cytochrome c oxidase, J Exp Biol 201:1163-75

Burke, P. V., Raitt, D. C., Allen, L. A., Kellogg, E. A., Poyton, R. O. (1997) Effects of oxygen concentration on the expression of cytochrome $\mathrm{c}$ and cytochrome $\mathrm{c}$ oxidase genes in yeast, J Biol Chem 272:14705-12

Castresana, J., Lubben, M., Saraste, M., Higgins, D. G. (1994) Evolution of cytochrome oxidase, an enzyme older than atmospheric oxygen, EMBO J 13:2516-25

Chacinska, A., Koehler, C. M., Milenkovic, D., Lithgow, T., Pfanner, N. (2009) Importing mitochondrial proteins: machineries and mechanisms, Cell 138:628-44

Chacinska, A., Lind, M., Frazier, A. E., Dudek, J., Meisinger, C., Geissler, A., Sickmann, A., Meyer, H. E., Truscott, K. N., Guiard, B., Pfanner, N., Rehling, P. (2005) Mitochondrial presequence translocase: switching between TOM tethering and motor recruitment involves Tim21 and Tim17, Cell120:817-29

Chacinska, A., van der Laan, M., Mehnert, C. S., Guiard, B., Mick, D. U., Hutu, D. P., Truscott, K. N., Wiedemann, N., Meisinger, C., Pfanner, N., Rehling, P. (2010) Distinct forms of mitochondrial TOM-TIM supercomplexes define signal-dependent states of preprotein sorting, Mol Cell Biol 30:307-18

Chan, D. C. (2006) Mitochondria: dynamic organelles in disease, aging, and development, Cell 125:1241-52

Chen, Y. C., Taylor, E. B., Dephoure, N., Heo, J. M., Tonhato, A., Papandreou, I., Nath, N., Denko, N. C., Gygi, S. P., Rutter, J. (2012) Identification of a protein mediating respiratory supercomplex stability, Cell Metab 15:34860

Claypool, S. M., Boontheung, P., McCaffery, J. M., Loo, J. A., Koehler, C. M. (2008) The cardiolipin transacylase, tafazzin, associates with two distinct respiratory components providing insight into Barth syndrome, Mol Biol Cell 19:5143-55

Cobine, P. A., Pierrel, F., Bestwick, M. L., Winge, D. R. (2006) Mitochondrial matrix copper complex used in metallation of cytochrome oxidase and superoxide dismutase, J Biol Chem 281:36552-9

Crofts, A. R. (2004) The cytochrome bc1 complex: function in the context of structure, Annu Rev Physiol 66:689733

Cruciat, C. M., Brunner, S., Baumann, F., Neupert, W., Stuart, R. A. (2000) The cytochrome bc1 and cytochrome c oxidase complexes associate to form a single supracomplex in yeast mitochondria, J Biol Chem 275:18093-8

D'Aurelio, M., Gajewski, C. D., Lenaz, G., Manfredi, G. (2006) Respiratory chain supercomplexes set the threshold for respiration defects in human mtDNA mutant cybrids, Hum Mol Genet 15:2157-69

Dagsgaard, C., Taylor, L. E., O'Brien, K. M., Poyton, R. O. (2001) Effects of anoxia and the mitochondrion on expression of aerobic nuclear COX genes in yeast: evidence for a signaling pathway from the mitochondrial genome to the nucleus, J Biol Chem 276:7593-601 
Decoster, E., Simon, M., Hatat, D., Faye, G. (1990) The MSS51 gene product is required for the translation of the COX1 mRNA in yeast mitochondria, Mol Gen Genet 224:111-8

Denko, N., Schindler, C., Koong, A., Laderoute, K., Green, C., Giaccia, A. (2000) Epigenetic regulation of gene expression in cervical cancer cells by the tumor microenvironment, Clin Cancer Res 6:480-7

Diaz, F., Fukui, H., Garcia, S., Moraes, C. T. (2006) Cytochrome c oxidase is required for the assembly/stability of respiratory complex I in mouse fibroblasts, Mol Cell Biol 26:4872-81

Dienhart, M. K., Stuart, R. A. (2008) The yeast Aac2 protein exists in physical association with the cytochrome bc1-COX supercomplex and the TIM23 machinery, Mol Biol Cell 19:3934-43

DiMauro, S., Schon, E. A. (2008) Mitochondrial disorders in the nervous system, Annu Rev Neurosci 31:91-123

Dudkina, N. V., Eubel, H., Keegstra, W., Boekema, E. J., Braun, H. P. (2005) Structure of a mitochondrial supercomplex formed by respiratory-chain complexes I and III, Proc Natl Acad Sci U S A 102:3225-9

Dudkina, N. V., Heinemeyer, J., Sunderhaus, S., Boekema, E. J., Braun, H. P. (2006) Respiratory chain supercomplexes in the plant mitochondrial membrane, Trends Plant Sci 11:232-40

Dudkina, N. V., Kudryashev, M., Stahlberg, H., Boekema, E. J. (2011) nteraction of complexes I, III, and IV within the bovine respirasome by single particle cryoelectron tomography, Proc Natl Acad Sci U S A 108:15196-200

Dyall, S. D., Brown, M. T., Johnson, P. J. (2004) Ancient invasions: from endosymbionts to organelles, Science 304:253-7

Endo, T., Yamano, K. (2010) Transport of proteins across or into the mitochondrial outer membrane, Biochim Biophys Acta 1803:706-14

Eubel, H., Heinemeyer, J., Braun, H. P. (2004) Identification and characterization of respirasomes in potato mitochondria, Plant Physiol 134:1450-9

Eubel, H., Jansch, L., Braun, H. P. (2003) New insights into the respiratory chain of plant mitochondria. Supercomplexes and a unique composition of complex II, Plant Physiol 133:274-86

Fansler B, Lowenstein J. M. (1969) Aconitase from pig heart, Methods in Enzymology 13:26-30

Fernandez-Vizarra, E., Tiranti, V., Zeviani, M. (2009) Assembly of the oxidative phosphorylation system in humans: what we have learned by studying its defects, Biochim Biophys Acta 1793:200-11

Finkel, T. (2011) Signal transduction by reactive oxygen species, J Cell Biol 194:7-15

Fontanesi, F., Soto, I. C., Barrientos, A. (2008) Cytochrome c oxidase biogenesis: new levels of regulation, IUBMB Life 60:557-68

Fontanesi, F., Soto, I. C., Horn, D., Barrientos, A. (2006) Assembly of mitochondrial cytochrome c-oxidase, a complicated and highly regulated cellular process, Am J Physiol Cell Physiol 291:C1129-47

Frazier, A. E., Taylor, R. D., Mick, D. U., Warscheid, B., Stoepel, N., Meyer, H. E., Ryan, M. T., Guiard, B., Rehling, P. (2006) Mdm38 interacts with ribosomes and is a component of the mitochondrial protein export machinery, J Cell Biol 172:553-64

Fukuda, R., Zhang, H., Kim, J. W., Shimoda, L., Dang, C. V., Semenza, G. L. (2007) HIF-1 regulates cytochrome oxidase subunits to optimize efficiency of respiration in hypoxic cells, Cell 129:111-22

Galati, D., Srinivasan, S., Raza, H., Prabu, S. K., Hardy, M., Chandran, K., Lopez, M., Kalyanaraman, B., Avadhani, N. G. (2009) Role of nuclear-encoded subunit Vb in the assembly and stability of cytochrome $c$ oxidase complex: implications in mitochondrial dysfunction and ROS production, Biochem J 420:439-49

Gietz, R. D., Schiestl, R. H. (2007) High-efficiency yeast transformation using the LiAc/SS carrier DNA/PEG method, Nat Protoc 2:31-4 
Giorgio, M., Migliaccio, E., Orsini, F., Paolucci, D., Moroni, M., Contursi, C., Pelliccia, G., Luzi, L., Minucci, S., Marcaccio, M., Pinton, P., Rizzuto, R., Bernardi, P., Paolucci, F., Pelicci, P. G. (2005) Electron transfer between cytochrome $\mathrm{c}$ and p66Shc generates reactive oxygen species that trigger mitochondrial apoptosis, Cell 122:22133

Glick, B. S., Brandt, A., Cunningham, K., Muller, S., Hallberg, R. L., Schatz, G. (1992) Cytochromes c1 and b2 are sorted to the intermembrane space of yeast mitochondria by a stop-transfer mechanism, Cell 69:809-22

Graier, W. F., Frieden, M., Malli, R. (2007) Mitochondria and $\mathrm{Ca}(2+)$ signaling: old guests, new functions, Pflugers Arch 455:375-96

Grandier-Vazeille, X., Bathany, K., Chaignepain, S., Camougrand, N., Manon, S., Schmitter, J. M. (2001) Yeast mitochondrial dehydrogenases are associated in a supramolecular complex, Biochemistry 40:9758-69

Graslund, S., Eklund, M., Falk, R., Uhlen, M., Nygren, P. A., Stahl, S. (2002) A novel affinity gene fusion system allowing protein A-based recovery of non-immunoglobulin gene products, J Biotechnol 99:41-50

Gray, M. W., Burger, G., Lang, B. F. (2001) The origin and early evolution of mitochondria, Genome Biol 2:REVIEWS1018

Hackenbrock, C. R., Chazotte, B., Gupte, S. S. (1986) The random collision model and a critical assessment of diffusion and collision in mitochondrial electron transport, J Bioenerg Biomembr 18:331-68

Harner, M., Korner, C., Walther, D., Mokranjac, D., Kaesmacher, J., Welsch, U., Griffith, J., Mann, M., Reggiori, F., Neupert, W. (2011) The mitochondrial contact site complex, a determinant of mitochondrial architecture, EMBO J 30:4356-70

Heinemeyer, J., Braun, H. P., Boekema, E. J., Kouril, R. (2007) A structural model of the cytochrome C reductase/oxidase supercomplex from yeast mitochondria, J Biol Chem 282:12240-8

Helbig, A. O., de Groot, M. J., van Gestel, R. A., Mohammed, S., de Hulster, E. A., Luttik, M. A., Daran-Lapujade, P., Pronk, J. T., Heck, A. J., Slijper, M. (2009) A three-way proteomics strategy allows differential analysis of yeast mitochondrial membrane protein complexes under anaerobic and aerobic conditions, Proteomics 9:478798

Hell, K., Neupert, W., Stuart, R. A. (2001) Oxa1p acts as a general membrane insertion machinery for proteins encoded by mitochondrial DNA, EMBO J 20:1281-8

Herrmann, J. M., Funes, S. (2005) Biogenesis of cytochrome oxidase-sophisticated assembly lines in the mitochondrial inner membrane, Gene 354:43-52

Hess, D. C., Myers, C. L., Huttenhower, C., Hibbs, M. A., Hayes, A. P., Paw, J., Clore, J. J., Mendoza, R. M., Luis, B. S., Nislow, C., Giaever, G., Costanzo, M., Troyanskaya, O. G., Caudy, A. A. (2009) Computationally driven, quantitative experiments discover genes required for mitochondrial biogenesis, PLoS Genet 5:e1000407

Hill, K., Model, K., Ryan, M. T., Dietmeier, K., Martin, F., Wagner, R., Pfanner, N. (1998) Tom40 forms the hydrophilic channel of the mitochondrial import pore for preproteins, Nature 395:516-21

Hoppins, S., Collins, S. R., Cassidy-Stone, A., Hummel, E., Devay, R. M., Lackner, L. L., Westermann, B., Schuldiner, M., Weissman, J. S., Nunnari, J. (2011) A mitochondrial-focused genetic interaction map reveals a scaffold-like complex required for inner membrane organization in mitochondria, J Cell Biol 195:323-40

Horan, S., Bourges, I., Taanman, J. W., Meunier, B. (2005) Analysis of COX2 mutants reveals cytochrome oxidase subassemblies in yeast, Biochem $\mathrm{J}$ 390:703-8

Hunte, C., Koepke, J., Lange, C., Rossmanith, T., Michel, H. (2000) Structure at 2.3 A resolution of the cytochrome bc(1) complex from the yeast Saccharomyces cerevisiae co-crystallized with an antibody $\mathrm{Fv}$ fragment, Structure 8:669-84

Hunte, C., Zickermann, V., Brandt, U. (2010) Functional modules and structural basis of conformational coupling in mitochondrial complex I, Science 329:448-51 
Ito, H., Fukuda, Y., Murata, K., Kimura, A. (1983) Transformation of intact yeast cells treated with alkali cations, J Bacteriol 153:163-8

Ivan, M., Kondo, K., Yang, H., Kim, W., Valiando, J., Ohh, M., Salic, A., Asara, J. M., Lane, W. S., Kaelin, W. G., Jr. (2001) HIFalpha targeted for VHL-mediated destruction by proline hydroxylation: implications for O2 sensing, Science 292:464-8

Iwata, S., Lee, J. W., Okada, K., Lee, J. K., Iwata, M., Rasmussen, B., Link, T. A., Ramaswamy, S., Jap, B. K. (1998) Complete structure of the 11-subunit bovine mitochondrial cytochrome bc1 complex, Science 281:64-71

Jia, L., Dienhart, M., Schramp, M., McCauley, M., Hell, K., Stuart, R. A. (2003) Yeast Oxa1 interacts with mitochondrial ribosomes: the importance of the C-terminal region of Oxa1, EMBO J 22:6438-47

Khalimonchuk, O., Bestwick, M., Meunier, B., Watts, T. C., Winge, D. R. (2010) Formation of the redox cofactor centers during Cox1 maturation in yeast cytochrome oxidase, Mol Cell Bio 30:1004-17

Krause, F., Reifschneider, N. H., Goto, S., Dencher, N. A. (2005) Active oligomeric ATP synthases in mammalian mitochondria, Biochem Biophys Res Commun 329:583-90

Kwast, K. E., Burke, P. V., Poyton, R. O. (1998) Oxygen sensing and the transcriptional regulation of oxygenresponsive genes in yeast, J Exp Biol 201:1177-95

Laemmli, U. K. (1970) Cleavage of structural proteins during the assembly of the head of bacteriophage T4, Nature 227:680-5

Lange, C., Hunte, C. (2002) Crystal structure of the yeast cytochrome bc1 complex with its bound substrate cytochrome c, Proc Natl Acad Sci U S A 99:2800-5

Lazarou, M., Smith, S. M., Thorburn, D. R., Ryan, M. T., McKenzie, M. (2009) Assembly of nuclear DNA-encoded subunits into mitochondrial complex IV, and their preferential integration into supercomplex forms in patient mitochondria, FEBS J 276:6701-13

Lenaz, G., Genova, M. L. (2010) Structure and organization of mitochondrial respiratory complexes: a new understanding of an old subject, Antioxid Redox Signal 12:961-1008

Li, Y., D'Aurelio, M., Deng, J. H., Park, J. S., Manfredi, G., Hu, P., Lu, J., Bai, Y. (2007) An assembled complex IV maintains the stability and activity of complex I in mammalian mitochondria, J Biol Chem 282:17557-62

Li, Y., Park, J. S., Deng, J. H., Bai, Y. (2006) Cytochrome c oxidase subunit IV is essential for assembly and respiratory function of the enzyme complex, J Bioenerg Biomembr 38: 283-91

Lill, R., Muhlenhoff, U. (2005) Iron-sulfur-protein biogenesis in eukaryotes, Trends Biochem Sci 30:133-41

Luciano, P., Geli, V. (1996) The mitochondrial processing peptidase: function and specificity, Experientia 52:1077-82

Manthey, G. M., McEwen, J. E. (1995) The product of the nuclear gene PET309 is required for translation of mature mRNA and stability or production of intron-containing RNAs derived from the mitochondrial COX1 locus of Saccharomyces cerevisiae, EMBO J 14:4031-43

Mathieu, L., Marsy, S., Saint-Georges, Y., Jacq, C., Dujardin, G. (2011) A transcriptome screen in yeast identifies a novel assembly factor for the mitochondrial complex III, Mitochondrion 11:391-6

McKenzie, M., Lazarou, M., Thorburn, D. R., Ryan, M. T. (2006) Mitochondrial respiratory chain supercomplexes are destabilized in Barth Syndrome patients, J Mol Biol 361:462-9

Meinecke, M., Wagner, R. Kovermann, P., Guiard, B., Mick, D. U., Hutu, D. P., Voos, W., Truscott, K. N., Chacinska, A., Pfanner, N., Rehling, P. (2006) Tim50 maintains the permeability barrier of the mitochondrial inner membrane, Science 312:1523-6 
Meisinger, C., Pfanner, N., Truscott, K. N. (2006) Isolation of yeast mitochondria, Methods Mol Biol 313:33-9

Mell, O. C., Seibel, P., Kadenbach, B. (1994) Structural organisation of the rat genes encoding liver- and hearttype of cytochrome c oxidase subunit Vla and a pseudogene related to the COXVla-L cDNA, Gene 140:179-86

Mick, D. U., Fox, T. D., Rehling, P. (2011) Inventory control: cytochrome c oxidase assembly regulates mitochondrial translation, Nat Rev Mol Cell Biol 12:14-20

Mick, D. U., Vukotic, M., Piechura, H., Meyer, H. E., Warscheid, B., Deckers, M., Rehling, P. (2010) Coa3 and Cox14 are essential for negative feedback regulation of COX1 translation in mitochondria, J Cell Biol 191:141-54

Mick, D. U., Wagner, K., van der Laan, M., Frazier, A. E., Perschil, I., Pawlas, M., Meyer, H. E., Warscheid, B., Rehling, P. (2007) Shy1 couples Cox1 translational regulation to cytochrome c oxidase assembly, EMBO J 26:4347-58

Mitchell, P., Moyle, J. (1968) Proton translocation coupled to ATP hydrolysis in rat liver mitochondria, Eur J Biochem 4:530-9

Mokranjac, D., Sichting, M., Popov-Celeketic, D., Mapa, K., Gevorkyan-Airapetov, L., Zohary, K., Hell, K., Azem, A., Neupert, W. (2009) Role of Tim50 in the transfer of precursor proteins from the outer to the inner membrane of mitochondria, Mol Biol Cell 20:1400-7

Moran, M., Marin-Buera, L., Gil-Borlado, M. C., Rivera, H., Blazquez, A., Seneca, S., Vazquez-Lopez, M., Arenas, J., Martin, M. A., Ugalde, C. (2010) Cellular pathophysiological consequences of BCS1L mutations in mitochondrial complex III enzyme deficiency, Hum Mutat 31:930-41

Moreno-Lastres, D., Fontanesi, F., Garcia-Consuegra, I., Martin, M. A., Arenas, J., Barrientos, A., Ugalde, C. (2012) Mitochondrial complex I plays an essential role in human respirasome assembly, Cell Metab 15:324-35

Netter, P., Carignani, G., Jacq, C., Groudinsky, O., Clavilier, L., Slonimski, P. P. (1982) The cytochrome oxidase subunit I split gene in Saccharomyces cerevisiae: genetic and physical studies of the mtDNA segment encompassing the 'cytochrome b-homologous' intron, Mol Gen Genet 188:51-9

Nijtmans, L. G., Taanman, J. W., Muijsers, A. O., Speijer, D., Van den Bogert, C. (1998) Assembly of cytochrome-c oxidase in cultured human cells, Eur J Biochem 254:389-94

Ott, M., Herrmann, J. M. (2010) Co-translational membrane insertion of mitochondrially encoded proteins, Biochim Biophys Acta 1803:767-75

Ott, M., Prestele, M., Bauerschmitt, H., Funes, S., Bonnefoy, N., Herrmann, J. M. (2006) Mba1, a membraneassociated ribosome receptor in mitochondria, EMBO J 25:1603-10

Pan, Y. (2011) Mitochondria, reactive oxygen species, and chronological aging: a message from yeast, Exp Gerontol 46:847-52

Paumard, P., Vaillier, J., Coulary, B., Schaeffer, J., Soubannier, V., Mueller, D. M., Brethes, D., di Rago, J. P., Velours, J. (2002) The ATP synthase is involved in generating mitochondrial cristae morphology, EMBO J 21:221-30

Perez-Martinez, X., Broadley, S. A., Fox, T. D. (2003) Mss51p promotes mitochondrial Cox1p synthesis and interacts with newly synthesized Cox1p, EMBO J 22:5951-61

Perez-Martinez, X., Butler, C. A., Shingu-Vazquez, M., Fox, T. D. (2009) Dual functions of Mss51 couple synthesis of Cox1 to assembly of cytochrome c oxidase in Saccharomyces cerevisiae mitochondria, Mol Biol Cell 20: $4371-80$

Pfeiffer, K., Gohil, V., Stuart, R. A., Hunte, C., Brandt, U., Greenberg, M. L., Schagger, H. (2003) Cardiolipin stabilizes respiratory chain supercomplexes, J Biol Chem 278:52873-80

Pierrel, F., Bestwick, M. L., Cobine, P. A., Khalimonchuk, O., Cricco, J. A., Winge, D. R. (2007) Coa1 links the Mss51 post-translational function to Cox1 cofactor insertion in cytochrome c oxidase assembly, EMBO J 26:4335-46 
Poyton, R. O. (1999) Models for oxygen sensing in yeast: implications for oxygen-regulated gene expression in higher eukaryotes, Respir Physiol 115:119-33

Preuss, M., Leonhard, K., Hell, K., Stuart, R. A., Neupert, W., Herrmann, J. M. (2001) Mba1, a novel component of the mitochondrial protein export machinery of the yeast Saccharomyces cerevisiae, J Cell Biol 153:1085-96

Puig, O., Caspary, F., Rigaut, G., Rutz, B., Bouveret, E., Bragado-Nilsson, E., Wilm, M., Seraphin, B. (2001) The tandem affinity purification (TAP) method: a general procedure of protein complex purification, Methods 24:21829

Raha, S., Robinson, B. H. (2000) Mitochondria, oxygen free radicals, disease and ageing, Trends Biochem Sci 25:502-8

Ryan, M. T., Voos, W., Pfanner, N. (2001) Assaying protein import into mitochondria, Methods Cell Biol 65:189215

Saddar, S., Dienhart, M. K., Stuart, R. A. (2008) The F1F0-ATP synthase complex influences the assembly state of the cytochrome bc1-cytochrome oxidase supercomplex and its association with the TIM23 machinery, J Biol Chem 283:6677-86

Saraste, M. (1999) Oxidative phosphorylation at the fin de siecle, Science 283:1488-93

Schafer, E., Dencher, N. A., Vonck, J., Parcej, D. N. (2007) Three-dimensional structure of the respiratory chain supercomplex I1IIIIIV1 from bovine heart mitochondria, Biochemistry 46:12579-85

Schafer, E., Seelert, H., Reifschneider, N. H., Krause, F., Dencher, N. A., Vonck, J. (2006) Architecture of active mammalian respiratory chain supercomplexes, J Biol Chem 281:15370-5Schagger, H. (2001) Blue-native gels to isolate protein complexes from mitochondria, Methods Cell Biol 65:231-44

Schagger, H., de Coo, R., Bauer, M. F., Hofmann, S., Godinot, C., Brandt, U. (2004) Significance of respirasomes for the assembly/stability of human respiratory chain complex I, J Biol Chem 279:36349-53

Schagger, H., Pfeiffer, K. (2000) Supercomplexes in the respiratory chains of yeast and mammalian mitochondria, EMBO J 19:1777-83

Schagger, H., von Jagow, G. (1991) Blue native electrophoresis for isolation of membrane protein complexes in enzymatically active form, Anal Biochem 199:223-31

Schmidt, O., Pfanner, N., Meisinger, C. (2010) Mitochondrial protein import: from proteomics to functional mechanisms, Nat Rev Mol Cell Biol 11:655-67

Schulz, C., Lytovchenko, O., Melin, J., Chacinska, A., Guiard, B., Neumann, P., Ficner, R., Jahn, O., Schmidt, B., Rehling, P. (2011) Tim50's presequence receptor domain is essential for signal driven transport across the TIM23 complex, J Cell Biol 195:643-56

Scorrano, L. (2009) Opening the doors to cytochrome c: changes in mitochondrial shape and apoptosis, Int J Biochem Cell Biol 41:1875-83

Semenza, G. L. (2007) Oxygen-dependent regulation of mitochondrial respiration by hypoxia-inducible factor 1 , Biochem J 405:1-9

Sickmann, A., Reinders, J., Wagner, Y., Joppich, C., Zahedi, R., Meyer, H. E., Schonfisch, B., Perschil, I., Chacinska, A., Guiard, B., Rehling, P., Pfanner, N., Meisinger, C. (2003) The proteome of Saccharomyces cerevisiae mitochondria, Proc Natl Acad Sci U S A 100:13207-12

Sikorski, R. S., Hieter, P. (1989) A system of shuttle vectors and yeast host strains designed for efficient manipulation of DNA in Saccharomyces cerevisiae, Genetics 122:19-27

Smith, J. L., Zhang, H., Yan, J., Kurisu, G., Cramer, W. A. (2004) Cytochrome bc complexes: a common core of structure and function surrounded by diversity in the outlying provinces, Curr Opin Struct Biol 14:432-9

Soto, I. C., Fontanesi, F., Liu, J., Barrientos, A. (2011) Biogenesis and assembly of eukaryotic cytochrome c oxidase catalytic core, Biochim Biophys Acta 
Strogolova, V., Furness, A., Robb-McGrath, M., Garlich, J., Stuart, R. A. (2012) Rcf1 and Rcf2, Members of the Hypoxia-Induced Gene 1 Protein Family, Are Critical Components of the Mitochondrial Cytochrome bc1Cytochrome c Oxidase Supercomplex, Mol Cell Biol 32:1363-73

Stroh, A., Anderka, O., Pfeiffer, K., Yagi, T., Finel, M., Ludwig, B., Schagger, H. (2004) Assembly of respiratory complexes I, III, and IV into NADH oxidase supercomplex stabilizes complex I in Paracoccus denitrificans, J Biol Chem 279:5000-7

Stuart, R. A. (2008) Supercomplex organization of the oxidative phosphorylation enzymes in yeast mitochondria, J Bioenerg Biomembr 40:411-7

Szyrach, G., Ott, M., Bonnefoy, N., Neupert, W., Herrmann, J. M. (2003) Ribosome binding to the Oxa1 complex facilitates co-translational protein insertion in mitochondria, EMBO J 22:6448-57

Taanman, J. W., Capaldi, R. A. (1993) Subunit Vla of yeast cytochrome c oxidase is not necessary for assembly of the enzyme complex but modulates the enzyme activity. Isolation and characterization of the nuclear-coded gene, J Biol Chem 268:18754-61

Tamura, Y., Harada, Y., Shiota, T., Yamano, K., Watanabe, K., Yokota, M., Yamamoto, H., Sesaki, H., Endo, T. (2009) Tim23-Tim50 pair coordinates functions of translocators and motor proteins in mitochondrial protein import, J Cell Biol 184:129-41

Tello, D., Balsa, E., Acosta-lborra, B., Fuertes-Yebra, E., Elorza, A., Ordonez, A., Corral-Escariz, M., Soro, I., Lopez-Bernardo, E., Perales-Clemente, E., Martinez-Ruiz, A., Enriquez, J. A., Aragones, J., Cadenas, S., Landazuri, M. O. (2011) Induction of the mitochondrial NDUFA4L2 protein by HIF-1alpha decreases oxygen consumption by inhibiting Complex I activity, Cell Metab 14:768-79

Thomas, D., Bron, P., Weimann, T., Dautant, A., Giraud, M. F., Paumard, P., Salin, B., Cavalier, A., Velours, J., Brethes, D. (2008) Supramolecular organization of the yeast F1Fo-ATP synthase, Biol Cell 100:591-601

Trumpower, B. L. (1990) Cytochrome bc1 complexes of microorganisms, Microbiol Rev 54:101-29

Trumpower, B. L., Gennis, R. B. (1994) Energy transduction by cytochrome complexes in mitochondrial and bacterial respiration: the enzymology of coupling electron transfer reactions to transmembrane proton translocation, Annu Rev Biochem 63:675-716

Truscott, K. N., Kovermann, P., Geissler, A., Merlin, A., Meijer, M., Driessen, A. J., Rassow, J., Pfanner, N., Wagner, R. (2001) A presequence- and voltage-sensitive channel of the mitochondrial preprotein translocase formed by Tim23, Nat Struct Biol 8:1074-82

Tsukihara, T., Aoyama, H., Yamashita, E., Tomizaki, T., Yamaguchi, H., Shinzawa-Itoh, K., Nakashima, R., Yaono, R., Yoshikawa, S. (1995) Structures of metal sites of oxidized bovine heart cytochrome c oxidase at 2.8 A, Science 269:1069-74

Tsukihara, T., Aoyama, H., Yamashita, E., Tomizaki, T., Yamaguchi, H., Shinzawa-Itoh, K., Nakashima, R., Yaono, R., Yoshikawa, S. (1996) The whole structure of the 13-subunit oxidized cytochrome c oxidase at $2.8 \mathrm{~A}$, Science 272:1136-44

Tsukihara, T., Itoh-Shinzawa, K., Yoshikawa, S. (1996) Structures of metal centers of bovine heart cytochrome c oxidase, Tanpakushitsu Kakusan Koso 41:1353-62

Tzagoloff, A., Akai, A., Needleman, R. B. (1975) Assembly of the mitochondrial membrane system. Characterization of nuclear mutants of Saccharomyces cerevisiae with defects in mitochondrial ATPase and respiratory enzymes, J Biol Chem 250:8228-35

van der Laan, M., Meinecke, M., Dudek, J., Hutu, D. P., Lind, M., Perschil, I., Guiard, B., Wagner, R., Pfanner, N., Rehling, P. (2007) Motor-free mitochondrial presequence translocase drives membrane integration of preproteins, Nat Cell Biol 9:1152-9

van der Laan, M., Wiedemann, N., Mick, D. U., Guiard, B., Rehling, P., Pfanner, N. (2006) A role for Tim21 in membrane-potential-dependent preprotein sorting in mitochondria, Curr Biol 16:2271-6 
Velours, J., Dautant, A., Salin, B., Sagot, I., Brethes, D. (2009) Mitochondrial F1F0-ATP synthase and organellar internal architecture, Int J Biochem Cell Biol 41:1783-9

von der Malsburg, K., Muller, J. M., Bohnert, M., Oeljeklaus, S., Kwiatkowska, P., Becker, T., LoniewskaLwowska, A., Wiese, S., Rao, S., Milenkovic, D., Hutu, D. P., Zerbes, R. M., Schulze-Specking, A., Meyer, H. E., Martinou, J. C., Rospert, S., Rehling, P., Meisinger, C., Veenhuis, M., Warscheid, B., van der Klei, I. J., Pfanner, N., Chacinska, A., van der Laan, M. (2011) Dual role of mitofilin in mitochondrial membrane organization and protein biogenesis, Dev Cell 21:694-707

Wagner, K., Perschil, I., Fichter, C. D., van der Laan, M. (2010) Stepwise assembly of dimeric F(1)F(0)-ATP synthase in mitochondria involves the small F(o)-subunits $k$ and i, Mol Biol Cell 21:1494-504

Wagner, K., Rehling, P., Sanjuan Szklarz, L. K., Taylor, R. D., Pfanner, N., van der Laan, M. (2009) Mitochondrial F1Fo-ATP synthase: the small subunits e and $\mathrm{g}$ associate with monomeric complexes to trigger dimerization, $\mathrm{J}$ Mol Biol 392:855-61

Wallace, D. C. (2005) Mitochondria and cancer: Warburg addressed, Cold Spring Harb Symp Quant Biol 70:36374

Wallace, D. C. (2007) Why do we still have a maternally inherited mitochondrial DNA? Insights from evolutionary medicine, Annu Rev Biochem 76:781-821

Wang, Y., Mohsen, A. W., Mihalik, S. J., Goetzman, E. S., Vockley, J. (2010) Evidence for physical association of mitochondrial fatty acid oxidation and oxidative phosphorylation complexes, J Biol Chem 285:29834-41

Waterland, R. A., Basu, A., Chance, B., Poyton, R. O. (1991) The isoforms of yeast cytochrome c oxidase subunit $\mathrm{V}$ alter the in vivo kinetic properties of the holoenzyme, J Biol Chem 266:4180-6

Wiedemann, N., van der Laan, M., Hutu, D. P., Rehling, P., Pfanner, N. (2007) Sorting switch of mitochondrial presequence translocase involves coupling of motor module to respiratory chain, J Cell Biol 179:1115-22

Wittig, I., Schagger, H. (2008) Structural organization of mitochondrial ATP synthase, Biochim Biophys Acta 1777:592-8

Wurm, C. A., Suppanz, I. E., Stoldt, S., Jakobs, S. (2010) Rapid FIAsH labelling in the budding yeast Saccharomyces cerevisiae, J Microsc 240:6-13

Xia, D., Yu, C. A., Kim, H., Xia, J. Z., Kachurin, A. M., Zhang, L., Yu, L., Deisenhofer, J. (1997) Crystal structure of the cytochrome bc1 complex from bovine heart mitochondria, Science 277:60-6

Yaffe, M. P., Schatz, G. (1984) Two nuclear mutations that block mitochondrial protein import in yeast, Proc Natl Acad Sci U S A 61:4819-23

Yamaguchi, R., Andreyev, A., Murphy, A. N., Perkins, G. A., Ellisman, M. H., Newmeyer, D. D. (2007) Mitochondria frozen with trehalose retain a number of biological functions and preserve outer membrane integrity, Cell Death Differ 14:616-24

Yoshikawa, S., Choc, M. G., O'Toole, M. C., Caughey, W. S. (1977) An infrared study of CO binding to heart cytochrome c oxidase and hemoglobin A. Implications re O2 reactions, J Biol Chem 252:5498-508

Yoshikawa, S., Muramoto, K., Shinzawa-Itoh, K. (2011) Proton-pumping mechanism of cytochrome C oxidase, Annu Rev Biophys 40:205-23

Yoshikawa, S., Shinzawa-Itoh, K., Tsukihara, T. (1998) Crystal structure of bovine heart cytochrome c oxidase at 2.8 A resolution, J Bioenerg Biomembr 30:7-14

Young, J. C., Hoogenraad, N. J., Hartl, F. U. (2003) Molecular chaperones Hsp90 and Hsp70 deliver preproteins to the mitochondrial import receptor Tom70, Cell 112:41-50

Yu, F., White, S. B., Zhao, Q., Lee, F. S. (2001) HIF-1alpha binding to VHL is regulated by stimulus-sensitive proline hydroxylation, Proc Natl Acad Sci U S A 98:9630-5 
Zhang, M., Mileykovskaya, E., Dowhan, W. (2002) Gluing the respiratory chain together. Cardiolipin is required for supercomplex formation in the inner mitochondrial membrane, J Biol Chem 277:43553-6

Zhang, M., Mileykovskaya, E., Dowhan, W. (2005) Cardiolipin is essential for organization of complexes III and IV into a supercomplex in intact yeast mitochondria, J Biol Chem 280:29403-8

Zitomer, R. S., Lowry, C. V. (1992) Regulation of gene expression by oxygen in Saccharomyces cerevisiae, Microbiol Rev 56:1-11 


\section{Acknowledgments}

First and foremost, I would like to thank Prof. Dr. Peter Rehling for giving me the opportunity to work on this project as independently as I could, with full support from his side. I would like to thank him for having time for me whenever I needed guidance and for all advices he provided these past four years.

Moreover, I am indebted to my thesis committee members, Prof. Gerhard Braus and Prof. Dirk Görlich, for their support and helpful discussions.

I also have to thank to Prof. Bettina Warscheid and people from her group who had performed all mass spec analyses of my isolations; to Prof. Dörthe Katschinski and Dr. Anke Zieseniss for their contribution to this work and to Prof. Stefan Jakobs and Daniel Jans for IF analyses of my samples.

I am grateful to Dr. Markus Deckers and Dr. David Mick for all the help, advices and grateful discussions.

I would like to express deep appreciation to Jonathan Melin and Dr. Jan Dudek for critical reading and revision of my thesis. Special thank to Oleksandr Lytovchenko for introducing me to the world of calculations, for reading Materials and methods part and for being always ready to answer all my questions related or not to science.

I appreciate Fabian Ludewig for his excellent technical support, especially during the revision time of my manuscript.

I have to say "Thank you" to Christine Vollmer for being good friend and for solving all my problems with different German offices. Without her some things would be impossible, like finding the flat in Göttingen.

Thank to all my former and present colleagues for making stimulating and friendly working atmosphere during all these years.

I am grateful to my parents for their love and support, not just during the period of my $\mathrm{PhD}$, but also through all my life. I would also like to thank to my husband Milos for sacrifices he had to make in the name of science, for his support and constant attention throughout this time.

At the end I would like to dedicate this work to my brother, who although not so long gone, still feels as he never left. 


\section{Curriculum Vitae}

\section{Milena Vukotic}

Current address: Institut of Biochemistry II, Humboldtallee 23, D-37073 Göttingen

Nationality and date of birth: Serbian, 30 June 1982

\section{Education}

March 2008 - present Doctoral studies; Georg August University Göttingen, Faculty of Biology, Göttingen Graduate School for Neurosciences and Molecular Biosciences (GGNB)

Oct 2001 - Apr 2007 Undergraduate studies; University of Belgrade, Faculty of Biology, Department of Molecular Biology and Physiology, Major: Applied Biochemistry

Research experience

March 2008 - present Institute of Biochemistry II, Göttingen, Germany; doctoral project, investigating biogenesis and composition of the respiratory chain complexes in the lab of Peter Rehling

Jan 2008 - Feb 2008 Institute of Biochemistry and Molecular Biology, University of Freiburg, Germany; targeting, translocation and folding of mitochondrial proteins in the lab of Nikolaus Pfanner

Oct 2006 - March 2007 Institute of Haematology, Clinical Centre of Serbia, Cytogenetic Laboratory; graduate thesis project "Cytogenetic of Acute Promyelocytic Leukaemia, the study of karyotype $t(15 ; 17)(q 22 ; q 11-21)$ " in the lab of Vesna Djordjevic 
